\title{
Modeling and Control of an Autonomous Underwater Vehicle with Combined Foil/Thruster \\ Actuators
}

by

Michael V. Jakuba

Submitted to the Joint Program in Applied Ocean Science and

Engineering

in partial fulfillment of the requirements for the degree of

Master of Science in Mechanical Engineering

at the
MASSACHUSETTS INSTITUTE OF TECHNOLOGY BIOLOCICAL
and the

WOODS HOLE OCEANOGRAPHIC INSTITUTION

February 2003

(c) Michael V. Jakuba, MMIII. All rights reserved.

The author hereby grants to MIT and WHOI permission to reproduce and distribute publicly paper and electronic copies of this thesis document in whole or in part.

Author ................................................

Joint Program in Applied Ocean Science and Engineering Jan 14,2003

Certified by .

Dana R. Yoerger

Associate Scientist, WHOI

Thesis Supervisor

Accepted by . .

Ain A. Sonin

Chairman, Department Committee on Graduate Students 


\title{
Modeling and Control of an Autonomous Underwater Vehicle with Combined Foil/Thruster Actuators
}

\author{
by
}

\author{
Michael V. Jakuba
}

\author{
Submitted to the Joint Program in Applied Ocean Science and Engineering \\ on Jan 14, 2003, in partial fulfillment of the \\ requirements for the degree of \\ Master of Science in Mechanical Engineering
}

\begin{abstract}
The Sentry AUV represents a radical departure from conventional AUV design, particularly with respect to actuation. The vehicle's combined foil/thruster actuators have the potential to produce a vehicle both maneuverable in the veritcal plane and efficient in forward flight, well suited to survey work over rough topography. Capitalizing on this; however, requires an understanding of the vehicles dynamics.

In this work, we present the development and analysis of an analytic model of the Sentry AUV. Our goals were to develop a model sufficiently accurate in terms of the mission profile to identify critical vehicle behaviors influencing successful mission completion. The analytical vehicle model was developed with structural accuracy in mind, and under the requirement that it handle a large range of vertical plane velocities.

Our primary methodology for analysis was through the design of a linear controller, whose behavior was investigated in simulation and as implemented on a $\frac{1}{4}$ scale physical model. Based on decoupled linearized models for near-horizontal flight derived from the full non-linear model, classical linear controllers were designed and validated by simulation and implementation on the physical model. Closed loop simulations conducted at high angle of attack verified the vehicle's predicted maneuverability in the vertical plane. Ultimately we determined the vehicle's input structure limited the achievable performance of a classical linear controller.
\end{abstract}

Thesis Supervisor: Dana R. Yoerger

Title: Associate Scientist, WHOI 


\section{Acknowledgments}

I would like to thank my advisor, Dr. Dana Yoerger for his support and sage advice. Thanks are also due to Dr. Albert Bradley for guiding me towards a practical modeling solution, and for the use of ABE's joystick to fly the physical model. I also acknowledge Matthew Walter for his help in building the physical model.

To my parents to whom I owe everything, thank you, yet again. And finally, thank Rachel for your unfailing confidence in me. 


\section{Contents}

1 Introduction $\quad 15$

2 The Sentry Autonomous Underwater Vehicle 19

2.1 Vehicle Geometry . . . . . . . . . . . . . . . . . . . . . . 19

2.2 Vehicle Mass Properties . . . . . . . . . . . . . . . . 21

2.3 Mission Profile . . . . . . . . . . . . . . . . . 23

3 Equations of Motion 25

3.1 Coordinate Frames . . . . . . . . . . . . . . . . . . . . 25

3.2 Kinematics . . . . . . . . . . . . . . . . . 25

3.3 Rigid Body Dynamics . . . . . . . . . . . . . . 28

3.4 Complete Equations of Motion ................ 30

4 Hydrodynamics 33

4.1 Added Mass . . . . . . . . . . . . . . . . . . . . . . . . 34

4.1 .1 Body Added Mass . . . . . . . . . . . . . . . . 35

4.1 .2 Foil Added Mass . . . . . . . . . . . . . . . . 37

4.1 .3 Combined Added Mass . . . . . . . . . . . . . . 38

4.2 Strip-Theory Lift and Drag $\ldots \ldots \ldots \ldots$

4.2.1 Basic Results from Dimensional Analysis . . . . . . . . . 41

4.2 .2 Lift and Drag Coefficient Approximation . . . . . . . . . 42

4.2.3 Differential Lift and Drag of an Aerodynamic Section . . . . . 45

4.3 Quadratic Hydrodynamic Moments . . . . . . . . . . . . . . . 48

4.3.1 Center of Pressure Locations . . . . . . . . . . . . . 49

4.3.2 Differential Moment of an Aerodynamic Section . . . . . . 49

4.3 .3 Combined Lift and Drag . . . . . . . . . . . 50

4.3.4 Expanded Expressions for Quadratic Lift and Drag . . . . . 51

4.4 Longitudinal Hull Lift Coefficients . . . . . . . . . . . . . . 53 
4.4.1 Linear Damping . . . . . . . . . . . . . . . 56

4.5 Hydrostatic Forces . . . . . . . . . . . . . . . . . . . . . . 57

4.6 Thruster and Control Foil Interaction . . . . . . . . . . 58

5 Simplified Models $\quad 63$

5.1 General Linearization Procedure . . . . . . . . . . . . . . 63

5.2 Near Horizontal Flight . . . . . . . . . . . . . . . 65

5.2 .1 Decoupled Models . . . . . . . . . . . . . . . 68

5.3 Nonlinearity Considerations . . . . . . . . . . . . . . 72

5.3.1 Flow Separation: Foil Stall . . . . . . . . . . . . . 72

5.3 .2 Input Mapping Nonlinearity . . . . . . . . . . . 72

6 Controller Design $\quad 75$

6.1 Control Objective . . . . . . . . . . . . . . . 76

6.2 Decoupled Control Design . . . . . . . . . . . . . 76

6.2 .1 Speed Control . . . . . . . . . . . . . . . . . 77

6.2 .2 Pitch-Depth Control . . . . . . . . . . . . . . 77

6.2 .3 Heading Control $\ldots \ldots \ldots \ldots \ldots$

6.3 Controller Evaluation: Simulation and Experimental Results . . . . 88

6.3.1 Controller Performance at Nominal Operating Conditions ... 88

6.3 .2 Speed Dependence . . . . . . . . . . . . . . . . 92

6.3.3 Controller Performance at High Angles of Attack . . . . . . 94

6.4 Non-Linear Approaches . . . . . . . . . . . . . . . . . . . . 97

$\begin{array}{lll}7 & \text { Conclusions } & 101\end{array}$

7.1 Further Work . . . . . . . . . . . . . . . . . . 102

7.1 .1 Model Verification . . . . . . . . . . . . . . 102

7.1 .2 Control Design . . . . . . . . . . . . . . . 103

A Expressions for Arbitrary Control Foil Angles 105

A.1 Rigid Body Inertia . . . . . . . . . . . . . . . . . 105

A.2 Combined Added Mass . . . . . . . . . . . . . . . 106

A.3 Gravitational Restoring Moment . . . . . . . . . . . . . 107

$\begin{array}{ll}\text { B Physical Model } & 109\end{array}$

B.1 Similitude . . . . . . . . . . . . . . . . . . . . . . 109

B.2 Design . . . . . . . . . . . . . . . . . . . 110 


\section{List of Figures}

1-1 The Sentry vehicle $\ldots \ldots \ldots \ldots \ldots \ldots \ldots$

$2-1$ Sentry: top view. . . . . . . . . . . . . . . . . . 20

$2-2$ Sentry: side view. . . . . . . . . . . . . . . . 20

2-3 Multibeam Sonar Bathymetry Collected by ABE . . . . . . . . 24

3-1 Sentry AUV Body-Fixed, Aft Foil, and Inertial Coordinate Frames . . 26

4-1 Aerodynamic Section Definition . . . . . . . . . . . . 45

4-2 Classical Actuator Disk Analysis of a Propeller . . . . . . . . . . 59

$4-3$ Thruster and Control Foil Interaction . . . . . . . . . . . 60

$4-4$ Lift Gain vs. Thrust . . . . . . . . . . . . . . . . 62

6-1 Pitch-depth Eigenvalues vs. Nominal Forward Speed . . . . . . . . 78

6-2 Vehicle Response (Linear Model) vs. Control Foil Angle Ratio . . . . 81

6-3 Zeros of the Depth Transfer Function (Linear Model) vs. Control Foil Angle Ratio . . . . . . . . . . . . . . . . . . . . 82

6-4 Zero of the Pitch Transfer Function (Linear Model) vs. Control Foil Angle Ratio . . . . . . . . . . . . . . . . . . . 83

6-5 Vehicle Response to a Ramp-Smoothed Transition in Desired Depths. 89

6-6 Controller Performance: Physical Model . . . . . . . . . . . . . 91

6-7 Closed Loop Vehicle Response to Step Change in Desired Depth . . 93

6-8 Simulated Vehicle Response with Pitch Disturbance. . . . . . . . . . . . 95

6-9 Simulated Vehicle Response with Yaw Disturbance. . . . . . . . . 96

6-10 A Foils-Fixed Configuration Suitable for Low Speed Maneuvering. . . 98

B-1 $\frac{1}{4}$-Scale Physical Model of Sentry . . . . . . . . . . . . 110

B-2 Physical Model: Midplane . . . . . . . . . . . . . . . . . 111

B-3 Physical Model: Electronics Housing . . . . . . . . . . . . 112

B-4 Physical Model: Interface . . . . . . . . . . . . . . 113 


\section{List of Tables}

2.1 Sentry Hull Geometric Parameters . . . . . . . . . . . . . . . . . . 21

2.2 Sentry Foil Geometric Parameters . . . . . . . . . . . . . . . . . 21

2.3 Sentry Hull/Roots Mass Properties . . . . . . . . . . . . . . . . 22

2.4 Sentry Foil Mass Properties . . . . . . . . . . . . . . . . . . . . . 22

2.5 Combined Mass Properties (Foils Flat) . . . . . . . . . . . . 22

4.1 Body Added Mass Coefficients . . . . . . . . . . . . . . . . . . . . . . . . . . . . . . . . . .

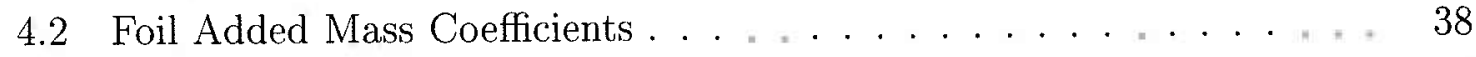

4.3 Combined Added Mass Coefficients $\left(\phi_{f}=0\right)$. . . . . . . . . . . 40

4.4 Geometry Relevant to Strip-Theory . . . . . . . . . . . . . . . . . 41

4.5 2D Section Lift and Drag Leading Coefficients . . . . . . . . . . . 42

4.6 Section CP Location (\% Chord) . . . . . . . . . . . . . . . . . . . . . . . . . . . . . . . . . . . .

4.7 Longitudinal Hull Lift Coefficients . . . . . . . . . . . . . . . . 56

5.1 Near Horizontal Flight: Combined and Mass Coefficients . . . . . . . 69

5.2 Near Horizontal Flight: Foil and Thruster Coefficients . . . . . . . . 69 


\section{Chapter 1}

\section{Introduction}

Over the last half decade, interest in autonomous underwater vehicles (AUVs) as tools for oceanographic science has grown. The Woods Hole Oceanographic's (WHOI) Autonomous Benthic Explorer (ABE) has been used with success to survey and collect data from deep ocean rift and hydrothermal vent sites. The data provided by an AUV for geological work represents a cost effective use of ship time. The data resolution is unmatched because of the vehicle's proximity to the seafloor, and the surface ship is free to perform other work while the AUV completes its survey. Ultimately, AUVs like ABE may be left entirely unattended awaiting ocean events or conducting repeated surveys.

$\mathrm{ABE}$ was originally designed to remain on the ocean floor for extended periods of time, periodically leaving a fixed mooring to collect data over the same region of the ocean. To date, ABE has not been used in that capacity, and has instead been deployed in conjunction with remotely operated vehicles and manned submersibles, generally surveying an area to locate features of interest before these other vehicles.

The time saved using ABE-generated maps to navigate along the seafloor results in a much more productive use of remotely operated and manned assets. In response to the pervasive use of $A B E$ as a complementary vehicle, a new AUV, Sentry, has been designed at WHOI, and is slated for sea trials in Spring 2003.

Sentry is radically different from all currently operational AUVs. It has been designed to incorporate the features of $\mathrm{ABE}$ that have made it well suited to scientific work, but its design is tailored to the particular mission that it will carry out [26]. Like ABE, Sentry will be highly maneuverable; capable of purely vertical motion and hover, and more hydrodynamically efficient than ABE. A larger battery capacity will allow longer mission times. Finally, experience with the $\mathrm{ABE}$ vehicle and particular attention to maintenance and operational considerations in the design ensures a quick 
deck turnaround to support daily operations.

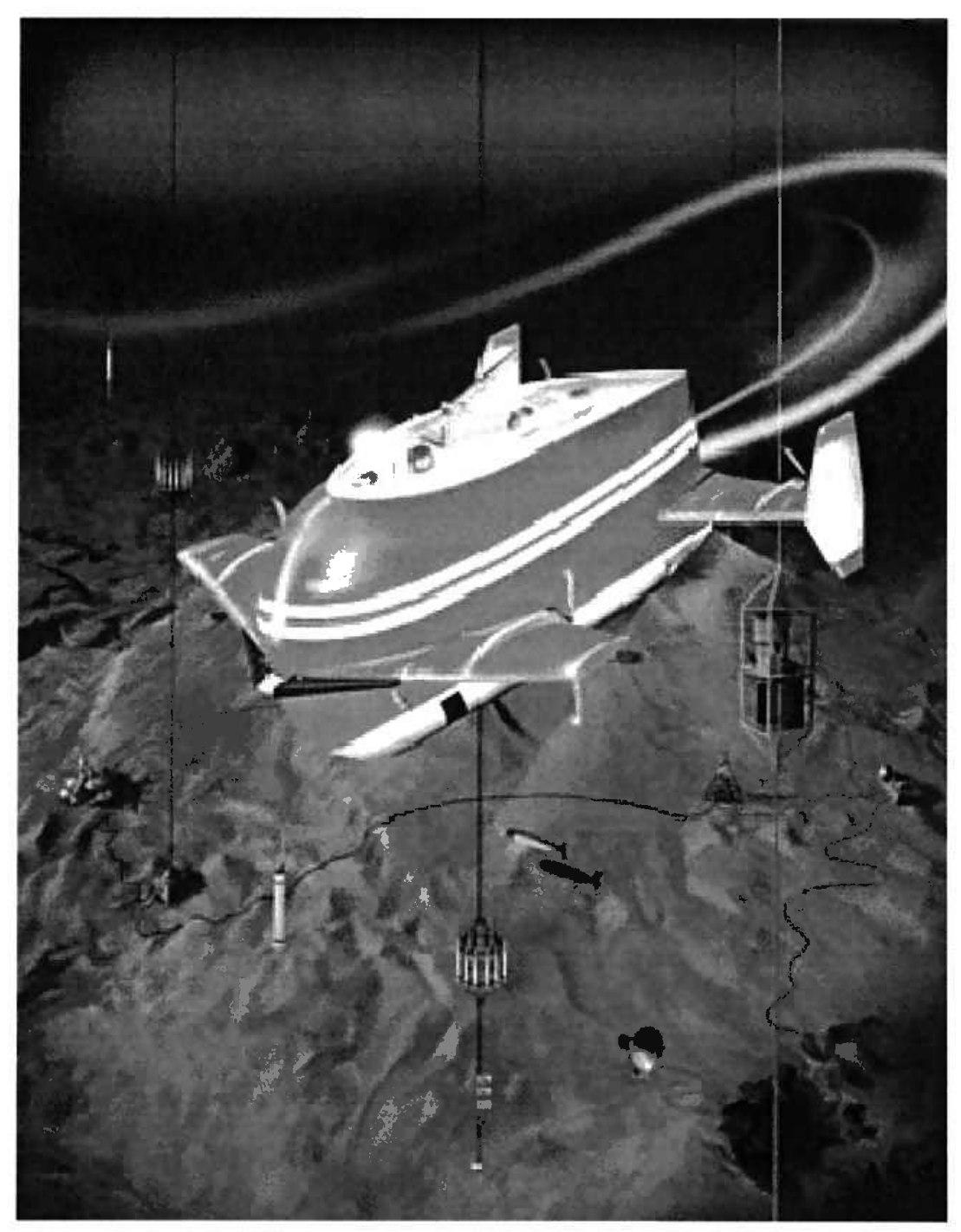

Figure 1-1: An artist's rendering of the Sentry vehicle. Both the fore and aft set of foils (and attached thrusters) are capable of swivelling $270^{\circ}$. Sentry will be both maneuverable and efficient in the vertical plane.

The proposed design shown in Figure 1-1 represents a departure from other AUV designs in three significant ways:

1. Unlike more standard AUV designs actuated by a single main propeller and aft control foils, Sentry will be maneuverable even at zero forward speed.

2. Sentry's swivelling thruster/foil actuators allow vehicle control uncompromised by thruster performance degradation in crossflows [22]. 
3. Sentry's aerodynamically shaped monolithic hull will provide the same degree of static stability in pitch and roll as possessed by the ABE vehicle, but at lower drag.

These design features represent hold considerable promise, but their realization will require a careful study of the proposed vehicle's dynamics and a control system design motivated by an understanding of the opportunities and constraints imposed by the vehicle dynamics. This analysis is the subject of this work.

The first part of this work (Chapters $3 \& 4$ ), is devoted to the development of a six-degree of freedom vehicle dynamics model. The model developed herein is based on theoretical and existing empirical hydrodynamics work. It is expected that parameters within the model will require tuning once the full scale vehicle is operational in Spring 2003; our focus herein is on model structure. A sufficiently accurate model structure and understanding of the resultant dynamics through analysis and simulation enables intelligent model-based control design, and ensures that once the full-scale vehicle becomes available, its dynamic behavior will be understood.

In the second part (Chapters $5 \& 6$ ), we present an analysis of near equilibrium flight conditions for nominally horizontal flight and a linear control design based upon this simplified model. Simulation results are presented using the full non-linear sixdegree of freedom model. Results are also given from the implementation of this control design on a $\frac{1}{4}$-scale physical model. We close by considering the limitations of linear control design in fully exploiting the capabilities of the Sentry vehicle and suggest directions for further work. 


\section{Chapter 2}

\section{The Sentry Autonomous Underwater Vehicle}

In this chapter we define the basic geometrical and mass properties of the Sentry vehicle. The dimensions and mass properties given are based on preliminary design data and may change as the vehicle nears completion.

\section{$2.1 \quad$ Vehicle Geometry}

A top and side view of the Sentry vehicle along with dimensions relevant to this work are shown in Figures 2-1 and 2-2, respectively.

Values for the symbolic dimensions shown in the figures are given in Tables 2.1 and 2.2. Foil area is large relative to body size to damp vehicle pitch and roll. The leading edge slope of the foils is motivated by the requirement that the vehicle shed lines from moorings passively. The large vertical foils protruding from the ends of the aft horizontal foils shown in Figure 1-1 are not shown above. These foils will probably not be on the real vehicle and are not included in the analytical model developed herein. 


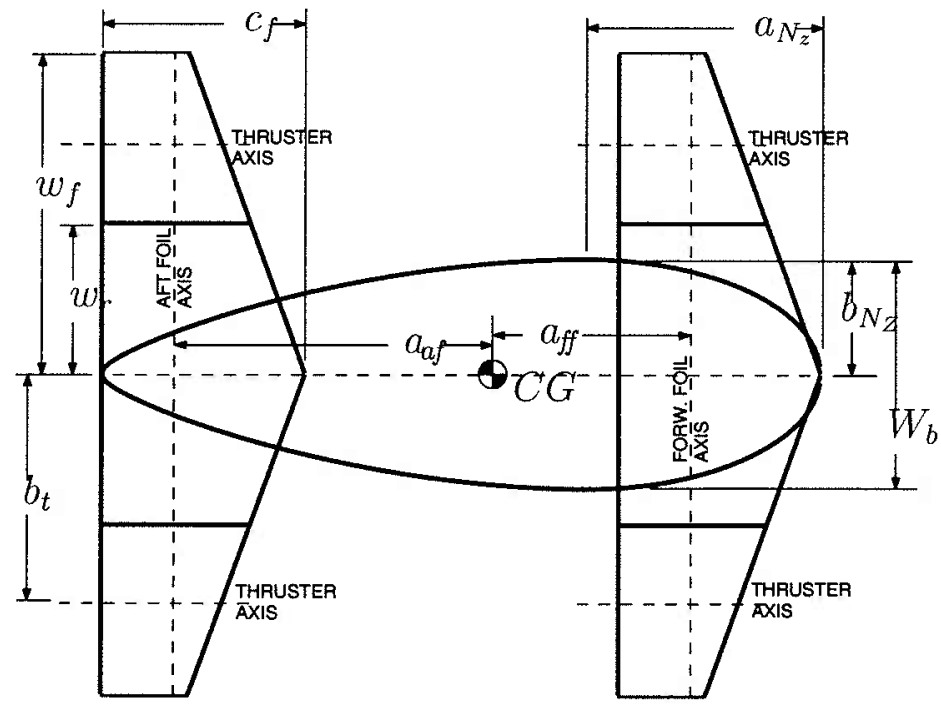

Figure 2-1: Sentry: top view.

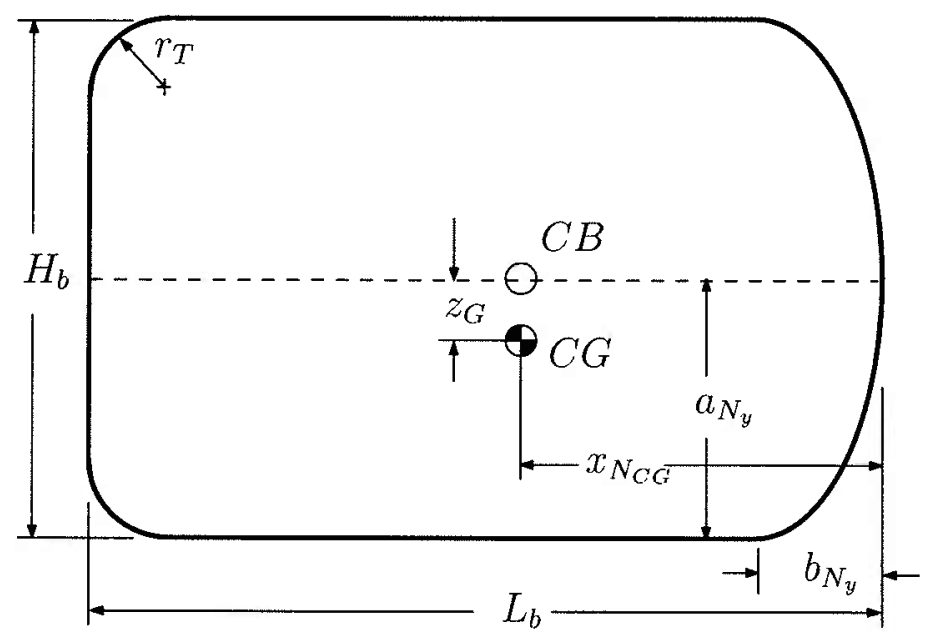

Figure 2-2: Sentry: side view. 
Table 2.1: Sentry Hull Geometric Parameters

\begin{tabular}{crll}
\hline \hline Parameter & Value & $\overline{\text { Units }}$ & Description \\
\hline$L_{b}$ & 2.53 & $\mathrm{~m}$ & vehicle length \\
$W_{b}$ & 0.81 & $\mathrm{~m}$ & vehicle width \\
$H_{b}$ & 1.67 & $\mathrm{~m}$ & vehicle height \\
$a_{N_{z}}$ & 0.83 & $\mathrm{~m}$ & nose major axis in $x y$-plane \\
$b_{N_{s}}$ & 0.41 & $\mathrm{~m}$ & nose minor axis in $x y$-plane \\
$a_{N_{3}}$ & 0.84 & $\mathrm{~m}$ & nose major axis in $y z$-plane \\
$b_{N_{y}}$ & 0.41 & $\mathrm{~m}$ & nose minor axis in $y z$-plane \\
$r_{T}$ & 0.25 & $\mathrm{~m}$ & rounded tail radius \\
\hline \hline
\end{tabular}

Table 2.2: Sentry Foil Geometric Parameters

\begin{tabular}{crll}
\hline \hline Parameter & Value & Units & Description \\
\hline$\Lambda_{f}$ & 15 & deg & $\frac{1}{4}$-chord foil sweep angle \\
$w_{f}$ & 1.14 & $\mathrm{~m}$ & foil extent \\
$w_{r}$ & 0.53 & $\mathrm{~m}$ & root extent \\
$c_{f}$ & 0.71 & $\mathrm{~m}$ & projected chord length \\
$a_{f f}$ & 0.70 & $\mathrm{~m}$ & $x$-vector to fore foil axis \\
$a_{a f}$ & -1.12 & $\mathrm{~m}$ & $x$-vector to aft foil axis \\
$b_{t}$ & 0.81 & $\mathrm{~m}$ & thruster moment arm \\
\hline \hline
\end{tabular}

We will find it useful in later sections to refer to the vehicle as composed of discrete units: the vehicle hull, two sets of stationary foil roots, and two sets of control foils. Each set of control foils is defined as consisting of the pair of rotating foils, the shaft that connects them, and the two thrusters mounted on either foil.

\subsection{Vehicle Mass Properties}

The vehicle center of mass (CG) and center of buoyancy (CB) are as shown in Figure 22. The $\mathrm{CB}$ is located at the center of mass of a vehicle of identical geometric extent, but of uniform density. This location of the CB assumes that that any water entrained in the flooded hull of the vehicle acts as though it were rigidly attached to the rest of the vehicle, a reasonable assumption for the vehicle when moving at cruising speed. The vertical displacement between the $\mathrm{CB}$ and $\mathrm{CG}$ shown above is based on an estimate of the vehicle's mass distribution.

All foil parameters are rough estimates based on incomplete design data. 
Table 2.3: Sentry Hull/Roots Mass Properties

\begin{tabular}{crll}
\hline \hline Parameter & Value & Units & Description \\
\hline$V_{b}$ & 1.93 & $\mathrm{~m}^{3}$ & body volume including entrained water \\
$B_{b}$ & 18,960 & $\mathrm{~N}$ & body buoyancy force \\
$m_{b}$ & 1,890 & $\mathrm{~kg}$ & wet body mass \\
$W_{b}$ & 18,600 & $\mathrm{~N}$ & wet body weight \\
$x_{N_{C G}}$ & -1.16 & $\mathrm{~m}$ & x-vector from nose to CG \\
$z_{B}$ & 0.00 & $\mathrm{~m}$ & $z$-vector to CB \\
$z_{G}$ & 0.20 & $\mathrm{~m}$ & $z$-vector to CG \\
$I_{b_{x x}}$ & 589 & $\mathrm{~kg} \cdot \mathrm{m}^{2}$ & body moment of inertia \\
$I_{b_{y y}}$ & 1,830 & $\mathrm{~kg} \cdot \mathrm{m}^{2}$ & body moment of inertia \\
$I_{b_{z z}}$ & 1,380 & $\mathrm{~kg} \cdot \mathrm{m}^{2}$ & body moment of inertia \\
$I_{b_{x z}}$ & 50 & $\mathrm{~kg} \cdot \mathrm{m}^{2}$ & body product of inertia \\
\hline \hline
\end{tabular}

Table 2.4: Sentry Foil Mass Properties

\begin{tabular}{crll}
\hline \hline Parameter & Value & Units & Description \\
\hline$V_{f}$ & 1.9 & $\mathrm{~m}^{3}$ & foil volume including entrained water \\
$B_{f}$ & 0 & $\mathrm{~N}$ & foil buoyancy force \\
$m_{f}$ & 20 & $\mathrm{~kg}$ & wet foil mass \\
$W_{b}$ & 190 & $\mathrm{~N}$ & wet foil weight \\
$I_{f_{x x}}$ & 10 & $\mathrm{~kg} \cdot \mathrm{m}^{2}$ & foil moment of inertia \\
$I_{f_{y y}}$ & 100 & $\mathrm{~kg} \cdot \mathrm{m}^{2}$ & foil moment of inertia \\
$I_{f_{z z}}$ & 100 & $\mathrm{~kg} \cdot \mathrm{m}^{2}$ & foil moment of inertia \\
\hline \hline
\end{tabular}

Table 2.5: Combined Mass Properties (Foils Flat)

\begin{tabular}{crll}
\hline \hline Parameter & Value & Units & Description \\
\hline$V$ & 1.9 & $\mathrm{~m}^{3}$ & vehicle volume \\
$B$ & 18,960 & $\mathrm{~N}$ & vehicle buoyancy \\
$m$ & 1,930 & $\mathrm{~kg}$ & wet vehicle mass \\
$W$ & 18,960 & $\mathrm{~N}$ & wet vehicle weight \\
$I_{x x}$ & 790 & $\mathrm{~kg} \cdot \mathrm{m}^{2}$ & vehicle moment of inertia \\
$I_{y y}$ & 1890 & $\mathrm{~kg} \cdot \mathrm{m}^{2}$ & vehicle moment of inertia \\
$I_{z z}$ & 1610 & $\mathrm{~kg} \cdot \mathrm{m}^{2}$ & vehicle moment of inertia \\
$I_{x z}$ & 50 & $\mathrm{~kg} \cdot \mathrm{m}^{2}$ & vehicle product of inertia \\
\hline \hline
\end{tabular}




\subsection{Mission Profile}

The Sentry vehicle design is tailored to deep sea survey above topographically complex terrain. A model of the vehicle needs only to be accurate within the operational envelope defined by such missions.

Deep sea survey as performed by $\mathrm{ABE}[26,27,24,23]$ consists of a descent to a predetermined position on the sea floor followed by the execution of a series of lawn mower-like tracklines at constant height above the seafloor (typically $5 \mathrm{~m}$ to $100 \mathrm{~m}$ ), and finally an ascent back to the surface for recovery. An example of the tracklines followed by ABE during three July 2002 dives above the Explorer Ridge $\left(49^{\circ} 46^{\prime} \cdot \mathrm{N}, 130^{\circ} 16^{\prime} \cdot \mathrm{W}\right)$ are shown in Figure 2-3 superimposed above the bathymetric map generated using data collected during those dives. The bathymetry shown in Figure 2-3 is characterized by steep ridges running NNW and rapid changes of elevation as indicated by the depth scale on the right of the plot. This topography is typical of the regions where Sentry will operate. Thus the Sentry vehicle must be maneuverable in the vertical plane to successfully maintain a prescribed height above the bottom. As bottom-following bandwidth is crucial to the vehicle's performance, our model will will need to be accurate over a wide range of vertical and horizontal speeds.

The tracklines followed by ABE in the above example are identical in terms of general shape to those the Sentry vehicle will follow, although parameters such as trackline spacing vary according to the desired data product. These tracklines are straight, and deep-ocean near bottom currents are rare, thus limiting the lateral bandwidth required to successfully follow them. For this reason the Sentry vehicle is not equipped with lateral thrusters. Furthermore our model need only be accurate to low heading rates. 

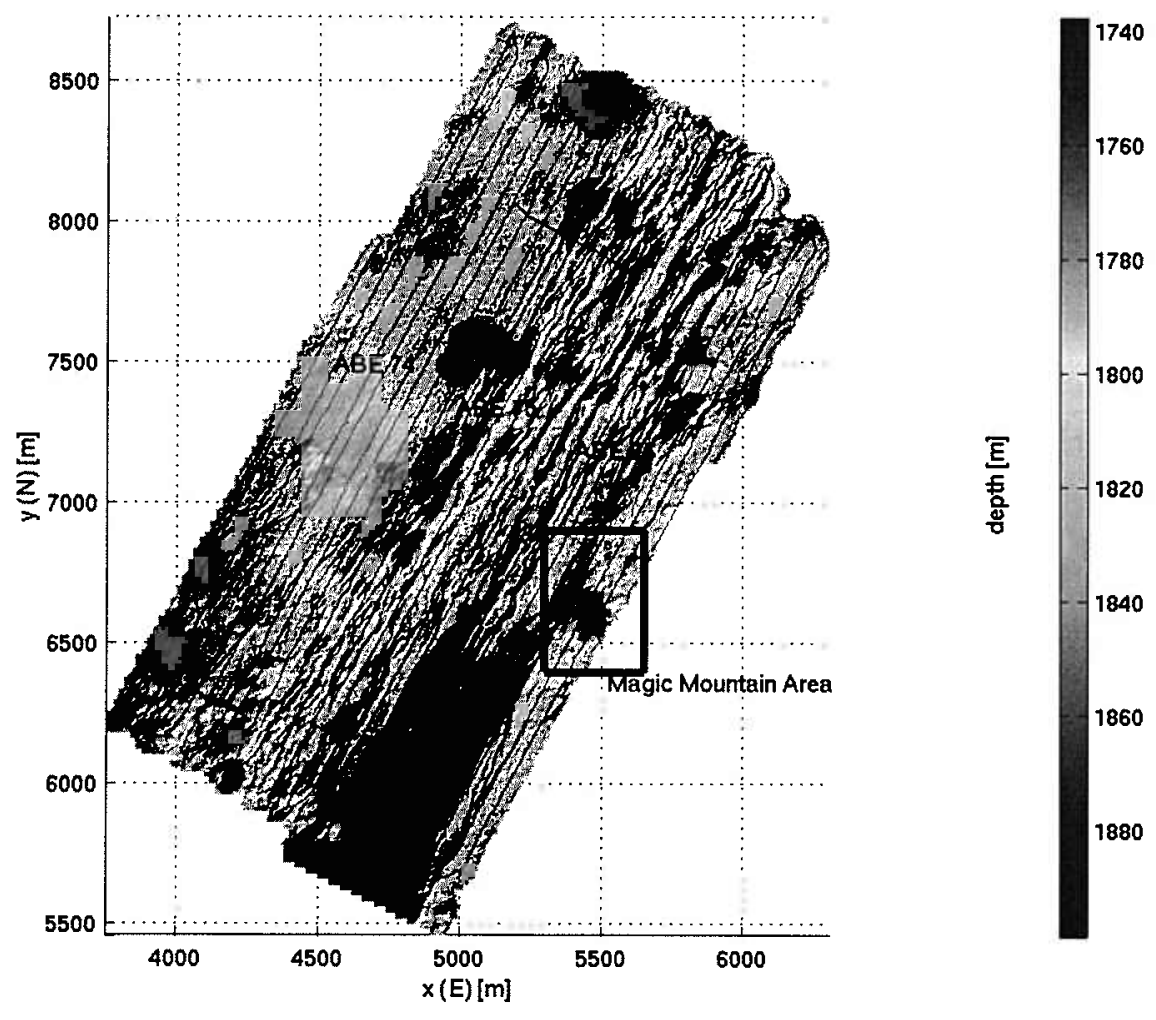

Figure 2-3: Multibeam sonar bathymetry collected by ABE over Explorer Ridge, July 2002. Superimposed ABE tracklines from three dives are shown running roughly NNW in a lawn mower-like pattern. The highlighted region encloses an area of active hydrothermal venting known as Magic Mountain in which over 30 vents were discovered with the aid of this bathymetry. Bathymetry data are shown interpolated onto a $2 \mathrm{~m}$ regular grid. 


\section{Chapter 3}

\section{Equations of Motion}

In this chapter, we define the governing equations of motion for the Sentry AUV. Explicit expressions for the hydrodynamic forces incident on the vehicle are given in Chapter 4.

\subsection{Coordinate Frames}

We approximate as inertial a frame fixed to the ocean surface. All vehicle motions are described relative to this frame. We further define a body-fixed coordinate frame fixed in the vehicle's body such that its origin is coincident with the vehicle's center of buoyancy when the control foils are in their neutral position. In addition, we will find it useful to define a third set of coordinate frames, fixed rigidly to each rotating pair of control foils. These frames are illustrated in Figure 3-1. The coordinate frame associated with the forward control foil is not shown. Coordinate directions for the inertial and body-fixed reference frames are consistent with convention for marine vehicles [5]. The coordinate directions in the foil frames are chosen consistent with our generalized strip-theory approach to the hydrodynamics of the control foils (Section 4.2).

\subsection{Kinematics}

The motion of the body-fixed and foil-fixed coordinate frames are described relative to the inertial frame. We denote the general state of motion of the body-fixed and foil-fixed coordinate frames relative to the inertial frame by the following quantities: 


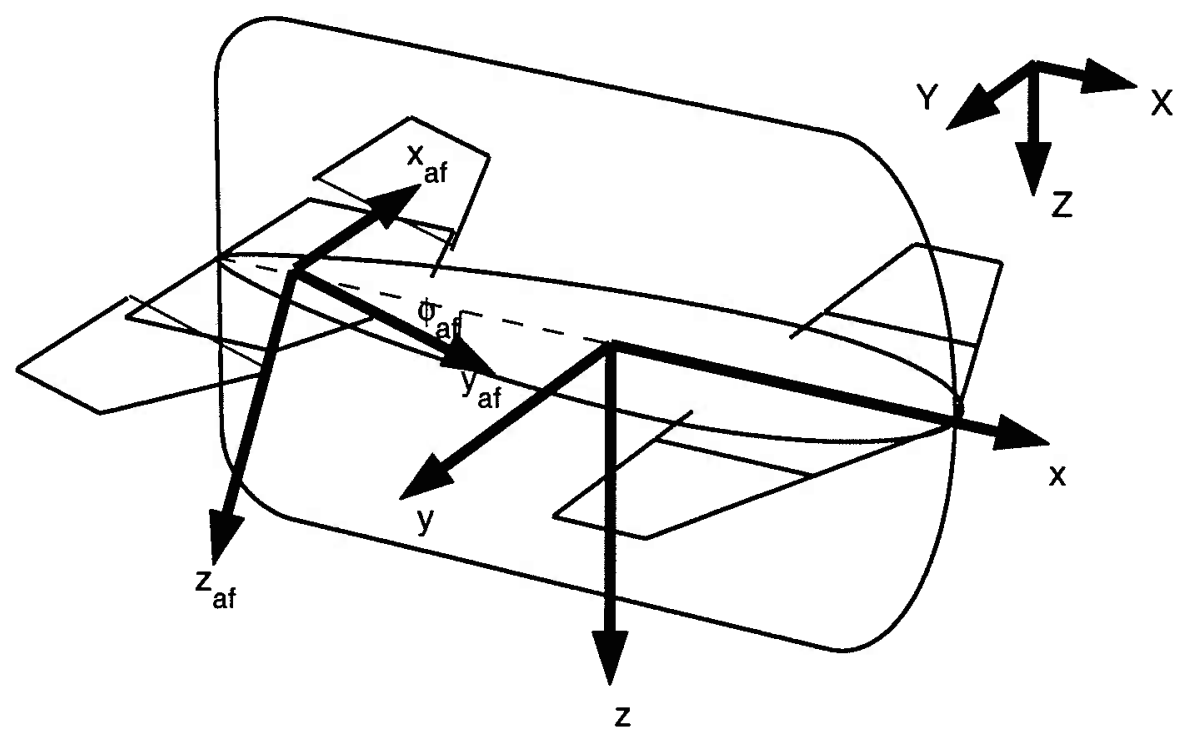

Figure 3-1: Sentry AUV Body-Fixed, Aft Foil, and Inertial Coordinate Frames

$$
\begin{aligned}
\boldsymbol{\eta}_{\mathbf{1}} & =[x, y, z]^{T} & & \text { inertial position } \\
\boldsymbol{\eta}_{\mathbf{2}} & =[\phi, \theta, \psi]^{T} & & \text { inertial orientation (Euler angles) } \\
\boldsymbol{\nu}_{\mathbf{1}} & =[u, v, w]^{T} & & \text { body-fixed linear velocity } \\
\boldsymbol{\nu}_{\mathbf{2}} & =[p, q, r]^{T} & & \text { body-fixed angular velocity } \\
\boldsymbol{\tau}_{1} & =[X, Y, Z]^{T} & & \text { external forces } \\
\boldsymbol{\tau}_{\mathbf{2}} & =[K, M, N]^{T} & & \text { external moments } \\
\phi_{f} & =\left[\phi_{f f}, \phi_{a f}\right]^{T} & & \text { foil angles } \\
\boldsymbol{h} & =\left[h_{f p}, h_{f s}, h_{a p}, h_{a s}\right]^{T} & & \text { thrust inputs }
\end{aligned}
$$

The above are consistent with Fossen's notation [5] with the exception of $\phi_{f}$ and $h$ which are particular to our vehicle.

Following the development found in Fossen [5], the translational velocity of the vehicle expressed in the body-fixed frame is expressed as linear velocity in the inertial frame through the transformation

$$
\dot{\boldsymbol{\eta}}_{1}=\boldsymbol{J}_{1}\left(\boldsymbol{\eta}_{\mathbf{2}}\right) \boldsymbol{\nu}_{1}
$$

where

$$
\boldsymbol{J}_{1}\left(\boldsymbol{\eta}_{2}\right)=\left[\begin{array}{ccc}
\cos \psi \cos \theta & -\sin \psi+\cos \psi \sin \theta \sin \phi & \sin \psi \sin \phi+\cos \psi \sin \theta \cos \phi \\
\sin \psi \cos \theta & \cos \psi \cos \phi+\sin \psi \sin \theta \sin \phi & -\cos \psi \sin \phi+\sin \psi \sin \theta \cos \phi \\
-\sin \theta & \cos \theta \sin \phi & \cos \theta \cos \phi
\end{array}\right] .
$$


The rotational velocity of the vehicle expressed in the body-fixed frame is expressed as rotational velocity in the inertial transformation through the transformation

$$
\dot{\eta}_{2}=\boldsymbol{J}_{2}\left(\boldsymbol{\eta}_{2}\right) \boldsymbol{\nu}_{2}
$$

where

$$
\boldsymbol{J}_{2}\left(\boldsymbol{\eta}_{2}\right)=\left[\begin{array}{ccc}
1 & \sin \phi \tan \theta & \cos \phi \tan \theta \\
0 & \cos \phi & -\sin \phi \\
0 & \sin \phi / \cos \theta & \cos \phi / \cos \theta
\end{array}\right]
$$

Note that $J_{2}$ above is singular for $\theta= \pm 90^{\circ}$. AUV Sentry is unlikely to ever pitch anywhere near $\pm 90^{\circ}$ while underway, and for this reason we chose to define the transformation matrices $\boldsymbol{J}_{1}$ and $\boldsymbol{J}_{2}$ in terms of familiar and widely used Euler angles, although other, singularity-free, transformations do exist.

Translational velocity vectors and geometric vectors defined in the foil-fixed frames are related to vectors defined in the body-fixed frame through the simple relation

$$
{ }^{b} r=\boldsymbol{R}_{f}{ }^{f} \boldsymbol{r}
$$

where the preceding superscript denotes the frame of the vector argument ( $b$ : body; $f$ : foil). Again employing Euler angles, the rotation matrix $R_{f}$ is given by

$$
\boldsymbol{R}_{f}=\left[\begin{array}{ccc}
0 & \cos \phi_{f} & -\sin \phi_{f} \\
-1 & 0 & 0 \\
0 & \sin \phi_{f} & \cos \phi_{f}
\end{array}\right]
$$

where the scalar angle $\phi_{f}$ denotes either the forward foil angle $\phi_{f f}$, or the aft foil angle $\phi_{a f}$, as appropriate. Note that although the foils are capable of $270^{\circ}$ rotation, $\boldsymbol{R}_{f}$ remains defined for all foil angles by the particular orientation of the foil-fixed frame relative to the foils' single axis of rotation. This choice of coordinate orientation is consistent with that of Section 4.2 , in which we derive a general equation for the lift and drag of a body composed of aerodynamically shaped two-dimensional sections. 


\subsection{Rigid Body Dynamics}

The six degree of freedom equations of motion for a general rigid body in vector form are [5]:

$$
\begin{aligned}
m\left(\left(\frac{\partial \boldsymbol{v}_{0}}{\partial t}\right)_{r e l}+\boldsymbol{\omega} \times \boldsymbol{v}_{0}+\dot{\boldsymbol{\omega}} \times \boldsymbol{r}_{G}+\boldsymbol{\omega} \times\left(\boldsymbol{\omega} \times \boldsymbol{r}_{G}\right)\right) & =\boldsymbol{f}_{0} \\
\boldsymbol{I}_{\mathbf{0}} \dot{\boldsymbol{\omega}}+\boldsymbol{\omega} \times\left(\boldsymbol{I}_{0} \boldsymbol{\omega}\right)+m \boldsymbol{r}_{\boldsymbol{G}} \times\left(\left(\frac{\partial \boldsymbol{v}_{0}}{\partial t}\right)_{r e l}+\boldsymbol{\omega} \times \boldsymbol{v}_{0}\right) & =\boldsymbol{m}_{\mathbf{0}}
\end{aligned}
$$

where $\boldsymbol{I}_{\mathbf{0}}$ denotes the inertia tensor defined at the origin of the body-fixed coordinate frame, and $r_{G}=\left[x_{G}, y_{G}, z_{G}\right]^{T}$ the vector from the frame origin to the center of gravity. Equation (3.7) represents the translational motion of the vehicle and (3.8) the rotational motion. Applying the notation defined in Section 3.2 to these equations and expanding yields:

$$
\begin{gathered}
m\left[\dot{u}-v r+w q-x_{G}\left(q^{2}+r^{2}\right)+y_{G}(p q-\dot{r})+z_{G}(p r+\dot{(q)})\right]=X \\
m\left[\dot{v}-w p+u r-y_{G}\left(r^{2}+p^{2}\right)+z_{G}(q r-\dot{p})+x_{G}(q p+\dot{(}(r))\right]=Y \\
\left.m\left[\dot{w}-u q+v p-z_{G}\left(p^{2}+q^{2}\right)+x_{G}(r p-\dot{q})+y_{G}(r q+\dot{(} p)\right)\right]=Z \\
I_{x x} \dot{p}+\left(I_{z z}-I_{y y}\right) q r-(\dot{r}+p q) I_{x z}+\left(r^{2}-q^{2}\right) I_{y z}+(p r-\dot{q}) I_{x y} \\
+m\left[y_{G}(\dot{w}-u q+v p)-z_{G}(\dot{v}-w p+u r)\right]=K \\
I_{y y} \dot{q}+\left(I_{x x}-I_{z z}\right) r p-(\dot{p}+q r) I_{x y}+\left(p^{2}-r^{2}\right) I_{z x}+(q p-\dot{r}) I_{y z} \\
+m\left[z_{G}(\dot{u}-v r+w q)-x_{G}(\dot{w}-u q+v p)\right]=M \\
I_{z z} \dot{p}+\left(I_{y y}-I_{x x}\right) p q-(\dot{q}+r p) I_{y z}+\left(q^{2}-p^{2}\right) I_{x y}+(r q-\dot{p}) I_{z x} \\
+m\left[x_{G}(\dot{v}-w p+u r)-y_{G}(\dot{u}-v r+w q)\right]=N .
\end{gathered}
$$

Several simplifications are possible by judicious placement of the body-fixed co-

ordinate frame. By placing the body-fixed reference frame at the center of buoyancy as defined in Section 3.1, and thus directly over the center of mass, $x_{G}=y_{G}=0$. Further, the mass symmetry of the vehicle across the $x-z$ plane implies the products of inertia $I_{x y}$ and $I_{y z}$ are both equal to zero, and the general rigid body inertia tensor reduces to:

$$
\boldsymbol{I}_{0}=\left[\begin{array}{ccc}
I_{x x} & 0 & I_{x z} \\
0 & I_{y y} & 0 \\
I_{x z} & 0 & I_{z z}
\end{array}\right]
$$


Thus, for the Sentry AUV, the rigid body equations of motion reduce to:

$$
\begin{aligned}
m\left[\dot{u}-v r+w q+z_{G}(p r+(q))\right] & =X \\
m\left[\dot{v}-w p+u r+z_{G}(q r-\dot{p})\right] & =Y \\
m\left[\dot{w}-u q+v p-z_{G}\left(p^{2}+q^{2}\right)\right] & =Z \\
I_{x x} \dot{p}+\left(I_{z z}-I_{y y}\right) q r-(\dot{r}+p q) I_{x z}-m\left[z_{G}(\dot{v}-w p+u r)\right] & =K \\
I_{y y} \dot{q}+\left(I_{x x}-I_{z z}\right) r p+\left(p^{2}-r^{2}\right) I_{z x}+m\left[z_{G}(\dot{u}-v r+w q)\right] & =M \\
I_{z z} \dot{p}+\left(I_{y y}-I_{x x}\right) p q+(r q-\dot{p}) I_{z x} & =N
\end{aligned}
$$

Equation (3.9) states the equations of motion for an ideal rigid body. For this equation and its simplified version (3.11) to apply, we approximate the foils, body, and any entrained water as a single rigid mass. Such approximations are consistent with typical marine vehicle models.

In this case, such an approximation may not be appropriate because of the large mass of the foils, and their large range of motion. In particular, the model does not account for forces and torques on the vehicle body induced by the rotation of foils relative to the vehicle body. Such a model could be constructed, but at the cost of the added complexity of two additional degrees of freedom (one for the single rotational degree of freedon of pair of foils).

Although such a model is tractable from a rigid body perspective $([2],[18])$, only extensive empirical work could yield a hydrodynamic model accurate enough to justify the added complexity (cf. [12]). Furthermore, since our eventual goal is control rather than modeling precision, it makes sense to leave the model in terms higherlevel control variables such as foil angles $\left(\phi_{f f}, \phi_{a f}\right)$ rather than the lower-level foil torques that would be required should the model order be increased.

Finally, as the foils rotate, the nominally static inertia tensor $\boldsymbol{I}_{\mathbf{0}}$ changes. It would violate conservation of energy to include this effect without increasing the model order, since such a model could add inertia arbitrarily and thereby increase kinetic energy without penalty. However, multiple nominally static configurations of the Sentry AUV are potentially of interest, and for this reason, Appendix A details the calculation of the vehicle's effective inertia matrix for arbitrary foil configuration.

The rigid body dynamics of the vehicle can be expressed in compact matrix form $[5]$ as

$$
M_{R B} \dot{\nu}+C_{R B}(\nu) \nu=\tau_{R B}
$$

where $M_{R B}$ is a positive definite matrix of inertial terms and $C_{R B}$ is a matrix of 
centripetal and Coriolis terms composed of vehicle velocities $\nu$ and the elements of $M_{R B}$

$$
\begin{aligned}
M_{R B}= & {\left[\begin{array}{cccccc}
m & 0 & 0 & 0 & m z_{G} & 0 \\
0 & m & 0 & -m z_{G} & 0 & 0 \\
0 & 0 & m & 0 & 0 & 0 \\
0 & -m z_{G} & 0 & I_{x x} & 0 & -I_{x z} \\
m z_{G} & 0 & 0 & 0 & I_{y y} & 0 \\
0 & 0 & 0 & -I_{x z} & 0 & I_{z z}
\end{array}\right] } \\
C_{R B}= & {\left[\begin{array}{ccccccc}
0 & 0 & 0 & m z_{G} r & m w & -m v \\
0 & 0 & 0 & -m w & m z_{G} r & m u \\
0 & 0 & 0 & m v-m z_{G} p & -m u-m z_{G} q & 0 \\
-m z_{G} r & m w & -m v+m z_{G} p & 0 & -I_{x z} p+I_{z z} r & -I_{y y} q \\
-m w & -m z_{G} r & m u+m z_{G} q & I_{x z} p-I_{z z} r & 0 & I_{x x} p-I_{x z} r \\
m v & -m u & 0 & I_{y y} q & -I_{x x} p+I_{x z} r & 0
\end{array}\right] }
\end{aligned}
$$

The vector $\boldsymbol{\tau}_{R B}$ in (3.12) represents all external forces.

The above formulations of $M_{R B}$ and $C_{R B}$ represent the rigid body dynamics for $\phi_{f f, a f}=0$. Formulations of these matrices for arbitrary foil angle are given in Appendix A. Note that $\boldsymbol{C}_{R B}$ has been formulated to be skew-symmetric, a fact potentially useful in control design $([20],[5])$.

\subsection{Complete Equations of Motion}

The complete non-linear six-degree of freedom equations of motion expressed in the body-fixed frame can be written in matrix form as [5]:

$$
\begin{gathered}
M \dot{\nu}+C(\nu) \nu+D\left(\nu, \phi_{f}\right) \nu+g(\eta)=b\left(\phi_{f}, h\right) \\
\dot{\eta}=J \nu
\end{gathered}
$$

where

$$
\begin{aligned}
M & =M_{R B}+M_{A} \\
C & =C_{R B}+C_{A} \\
D\left(\nu, \phi_{f}\right) & =D_{\text {s.t. }}\left(\nu, \phi_{f}\right)+D_{b . l .}+D_{\text {lin }}
\end{aligned}
$$

The component terms are described below.

The mass matrix $\boldsymbol{M}$ consists of the rigid body component $\boldsymbol{M}_{\boldsymbol{R} B}$ and an added mass component $\boldsymbol{M}_{\boldsymbol{A}}$. The centripetal and Coriolis force matrix $\boldsymbol{C}$ likewise consists of the rigid body component $\boldsymbol{C}_{\boldsymbol{R} \boldsymbol{B}}$ and an added mass component $\boldsymbol{C}_{\boldsymbol{A}}$. Both matrices of added mass terms are derived in Section 4.1.

The damping matrix $D$ consists of hydrodynamic lift and drag terms. Specifically, $\boldsymbol{D}_{\boldsymbol{s . t} \text {. }}$ covers the contribution of quadratic lift, drag and crossflow drag terms derived 
from a strip-theory treatment of the vehicle's hydrodynamics (Section 4.2). Under the assumption of foil/thruster independence (see Section 4.6), control foil lift and drag terms are included in this matrix. Longitudinal body lift forces inadequately estimated via strip-theory are added in a matrix of longitudinal body lift terms $\boldsymbol{D}_{b . l}$. (Section 4.4). Finally, linear drag terms important at low speeds are added in $\boldsymbol{D}_{\text {lin }}$ (Section 4.4.1).

The vector $\boldsymbol{g}(\boldsymbol{\eta})$ represents hydrostatic restoring forces (Section 4.5).

The thruster input vector $\boldsymbol{b}\left(\phi_{f}, \boldsymbol{h}\right)$ describes the mapping of each thruster onto the vehicle axes:

$$
\boldsymbol{b}\left(\phi_{f}, \boldsymbol{h}\right)=\left[\begin{array}{c}
\left(h_{f p}+h_{f s}\right) \cos \phi_{f f}+\left(h_{a p}+h_{a s}\right) \cos \phi_{a f} \\
\left(h_{f p}+h_{f s}\right) \sin \phi_{f f}+\left(h_{a p}+h_{a s}\right) \sin \phi_{a f} \\
b_{t}\left(\left(h_{f p}-h_{f s}\right) \sin \phi_{f f}+\left(h_{a p}-h_{a s}\right) \sin \phi_{a f}\right) \\
-a_{f f}\left(h_{f p}+h_{f s}\right) \sin \phi_{f f}-a_{a f}\left(h_{a p}+h_{a s s}\right) \sin \phi_{a f} \\
b_{t}\left(\left(h_{f p}-h_{f s}\right) \cos \phi_{f f}+\left(h_{a p}-h_{a s}\right) \cos \phi_{a f}\right)
\end{array}\right] .
$$

The mapping is trigonometrically dependent on the control foil angles $\phi_{\boldsymbol{f}}$. 


\section{Chapter 4}

\section{Hydrodynamics}

In this chapter we compute the hydrodynamic forces acting on the vehicle. The forces applied to the vehicle by the surrounding fluid medium can be roughly broken down into added mass, lift, drag, and hydrostatic forces. In all except the last, exact expressions would require analytic solutions to the Navier-Stokes equations with boundary conditions defined by the vehicle. In general this is intractable, and marine vehicle modelers have typically relied on Taylor series approximations to estimate hydrodynamic forces ([17]). For example, the axial force $X$ acting on a body moving at constant velocity in a fluid medium is well approximated by the familiar expression for quadratic drag:

$$
X=\frac{1}{2} \rho C_{D} A_{f}|u| u=X_{|u| u}|u| u
$$

This expression can be derived directly from dimensional analysis, or equivalently from a Taylor series expansion of the hydrodynamic forces incident upon the vehicle where only the most significant term has been kept. The subscript in the latter expression is consistent with standard notation [11] and denotes the partial derivative of the axial hydrodynamic force $X$ taken with respect to $|u| u$ :

$$
X_{|u| u}=\frac{\partial X}{\partial(|u| u)}
$$

The task of the modeler then becomes to analytically approximate or experimentally determine all coefficients of this form significant to the vehicle dynamics (cf. [10,6]). Usually, terms no greater than third order are required [1], although herein we consider only first and second order terms.

In this work, we follow this framework to model the added mass, linear drag, and longitudinal body lift forces, but take a slightly different although related approach 
to model most of the quadratic lift and drag forces acting on the vehicle. Rather than constant coefficients, we generalize the notion of crossflow integrals (see for example the vehicle models in [7] and [16]) to any body composed of aerodynamically shaped $2 \mathrm{D}$ sections oriented roughly into the oncoming flow. As these forces are necessarily expressed in integral form, explicit Taylor series coefficients cannot be derived.

This latter technique relies heavily on strip theory [see [14] for a general discussion]. Strip theory is predicated on the assumption that flow around a body of sufficient length to width ratio can be approximated as two dimensional except at the ends. The hydrodynamic forces on that body can then be approximated by integrating the force each two-dimensional section would experience if subjected to purely two-dimensional flow. We correct this approach for 3D effects by the appropriate choice of leading coefficients (cf. [13]).

The hydrostatic forces operating on the vehicle are straightforward, and discussed in Section 4.5 .

Finally, we present an argument based on a linearized analysis of the interaction between the thrusters and the foils to justify the independent treatment of thrust generated by the thrusters and foil lift.

Throughout this chapter, we will draw on mostly empirical and some theoretical results for the calculation of various leading coefficients, primarily from Hoerner's works [8,9], and those complied in [11]. In most cases, the geometry of the vehicle and foils given in Chapter 2 will also be required to arrive at numerical coefficient values. Numerical coefficient values are given at the end of each subsection.

\subsection{Added Mass}

The term "added mass" refers to pressure induced forces and moments proportional to the acceleration of the body[5]. Under the assumption that the added mass coefficients are constant, the added mass forces and moments $\tau_{A}$ can be expressed in terms of a symmetric positive definite added mass matrix $M_{A}$, and a skew symmetric matrix of centripetal and Coriolis added mass terms $C_{A}$ :

$$
M_{A} \dot{\nu}+C_{A}(\nu) \nu=\tau_{A}
$$

The matrix of centripetal and Coriolis terms $C_{A}$ is composed of the terms of $\boldsymbol{M}_{A}$ and the body-fixed velocities $\boldsymbol{\nu}$. Any one formulation of $\boldsymbol{C}_{A}$ is not unique, although it is always possible to formulate $\boldsymbol{C}_{A}$ such that it is skew-symmetric. See [5] for a 
theoretical derivation of this fact.

In the following sections we derive the specific form of $\boldsymbol{M}_{A}$ and $\boldsymbol{C}_{A}$ for the Sentry AUV. We consider the added mass of the body separately from that of the foils, before combining the results to reach an expression for the added mass forces incident on the complete vehicle.

\subsubsection{Body Added Mass}

Due to the top-bottom and port-starboard geometric symmetry of the body, the body added mass matrix reduces to:

$$
M_{A_{b}}=-\left[\begin{array}{cccccc}
X_{\dot{u}_{b}} & 0 & 0 & 0 & 0 & 0 \\
0 & Y_{\dot{v}_{b}} & 0 & 0 & 0 & N_{\dot{v}_{b}} \\
0 & 0 & Z_{\dot{w}_{b}} & 0 & M_{\dot{w}_{b}} & 0 \\
0 & 0 & 0 & K_{\dot{p}_{b}} & 0 & 0 \\
0 & 0 & M_{\dot{w}_{b}} & 0 & M_{\dot{q}_{b}} & 0 \\
0 & N_{\dot{v}_{b}} & 0 & 0 & 0 & N_{\dot{r}_{b}}
\end{array}\right]
$$

The expressions required to compute each element of $M_{A_{b}}$ above are given in the remainder of this section.

The axial added mass $X_{\dot{u}_{b}}$ can be approximated as the lateral added mass of a three-dimensional prolate ellipsoid with major semi-axis a equal to the vehicle's vertical extent, and minor axis $b$ equal to the vehicle's maximum width. Fossen [5] gives the formula for the lateral added mass of a prolate ellipsoid as:

$$
X_{u_{b}}=-\frac{4}{3} \pi \rho a b^{2} \frac{\beta_{0}}{2-\beta_{0}}
$$

where

$$
\beta_{0}=\frac{1}{e^{2}}-\frac{1-e^{2}}{2 e^{3}} \ln \frac{1+e}{1-e} \quad ; \quad e=1-(b / a)^{2}
$$

To estimate the remaining added mass coefficients in (4.2), we approximate the body as composed of geometrically simplified two-dimensional sections and integrate their two-dimensional added masses. Newman [14] gives the theoretical expressions 
for the added mass of a 2D ellipse with major axis $a$ and minor axis $b$ :

$$
\begin{aligned}
& m_{11}=\pi \rho b^{2} \\
& m_{22}=\pi \rho a^{2} \\
& m_{66}=\frac{1}{8} \pi \rho\left(a^{2}-b^{2}\right)^{2},
\end{aligned}
$$

The expressions for the added mass of a circle of radius $a$ with fins of extent $2 b$ are also given in [14]:

$$
\begin{aligned}
& m_{11}=\pi \rho\left[a^{2}+\left(b^{2}-a^{2}\right)^{2} / b^{2}\right] \\
& m_{22}=\pi \rho a^{2} \\
& m_{66}=\pi a^{4}\left(\pi^{-1} \csc ^{4} \alpha\left(2 \alpha^{2}-\alpha \sin 4 \alpha+\frac{1}{2} \sin ^{2} 2 \alpha\right)-\pi / 2\right) .
\end{aligned}
$$

where $\sin \alpha=2 a b /\left(a^{2}+b^{2}\right)$ and $\pi / 2<\alpha<\pi$.

Applying these expressions under the assumption that the body of the vehicle can be approximated to be of elliptical cross-section throughout, and as elliptical with protruding flat plates where the fixed foil roots protrude, the expressions for the remaining added mass terms can be written as:

$$
\begin{aligned}
Y_{\dot{v}_{b}} & =\pi \rho \int_{x_{b}} H_{b}(x)^{2} d x \\
Z_{\dot{w}_{b}} & =\pi \rho \int_{x_{b}}\left(\left(\frac{1}{2} W_{b}(x)\right)^{2}+\frac{\left(\left(\frac{1}{2} W_{t o t}(x)\right)^{2}-\left(\frac{1}{2} W_{b}(x)\right)^{2}\right)^{2}}{\frac{1}{2} W_{t o t}(x)^{2}}\right) d x \\
K_{\dot{p}_{b}} & =\frac{1}{8} \pi \rho \int x_{b}\left(\frac{1}{2} W_{t o t}\right)^{4} d x \\
M_{\dot{q}_{b}} & =\pi \rho \int_{x_{b}} x^{2}\left(\left(\frac{1}{2} W_{b}(x)\right)^{2}+\frac{\left(\left(\frac{1}{2} W_{t o t}(x)\right)^{2}-\left(\frac{1}{2} W_{b}(x)\right)^{2}\right)^{2}}{\frac{1}{2} W_{t o t}(x)^{2}}\right) d x \\
N_{\dot{r}_{b}} & =\pi \rho \int_{x_{b}} x^{2} H_{b}(x)^{2} d x \\
N_{\dot{v}_{b}} & =\pi \rho \int_{x_{b}} x H_{b}(x)^{2} d x \\
M_{\dot{w}_{b}} & =\pi \rho \int_{x_{b}} x\left(\left(\frac{1}{2} W_{b}(x)\right)^{2}+\frac{\left(\left(\frac{1}{2} W_{t o t}(x)\right)^{2}-\left(\frac{1}{2} W_{b}(x)\right)^{2}\right)^{2}}{\frac{1}{2} W_{\text {tot }}(x)^{2}}\right) d x
\end{aligned}
$$

where the terms $H_{b}(x), W_{b}(x)$, and $W_{t o t}(x)$ represent the height, centerline width, and width-including-roots of the body respectively.

The resultant numerical values for the added mass coefficients are given in Ta- 
ble 4.1 .

Table 4.1: Body Added Mass Coefficients

\begin{tabular}{crll}
\hline \hline Parameter & Value & Units & Description \\
\hline$X_{\dot{u}_{b}}$ & -366 & $\mathrm{~kg}$ & axial added mass \\
$Y_{\dot{v}_{b}}$ & -4960 & $\mathrm{~kg}$ & lateral added mass \\
$Z_{\dot{w}_{b}}$ & -1520 & $\mathrm{~kg}$ & vertical added mass \\
$K_{\dot{p}_{b}}$ & -41.9 & $\mathrm{~kg} \cdot \mathrm{m}^{2}$ & roll added mass \\
$M_{\dot{q}_{b}}$ & -690 & $\mathrm{~kg} \cdot \mathrm{m}^{2}$ & pitch added mass \\
$N_{\dot{r}_{b}}$ & -2210 & $\mathrm{~kg} \cdot \mathrm{m}^{2}$ & yaw added mass \\
$M_{\dot{w}_{b}}$ & -168 & $\mathrm{~kg} \cdot \mathrm{m}$ & added mass cross term \\
$N_{\dot{v}_{b}}$ & 816 & $\mathrm{~kg} \cdot \mathrm{m}$ & added mass cross term \\
\hline \hline
\end{tabular}

\subsubsection{Foil Added Mass}

Each pair of rotating foils is symmetric across the plane defined by the foil chords, symmetric across the plane perpendicular to the foil axis of rotation, and nearly symmetric across the plane perpendicular to the foil chords and coincident with the foil's rotational axis. Due to this symmetry, the mass matrix for either foil is diagonal. However, the small projected areas of each foil pair relative to the vehicle body onto all planes except the that parallel to the foil chords suggest that added mass terms associated with other planes can be neglected. Under this assumption, the added mass matrix of each foil pair reduces to:

$$
M_{A_{f}}=-\left[\begin{array}{cccccc}
0 & 0 & 0 & 0 & 0 & 0 \\
0 & 0 & 0 & 0 & 0 & 0 \\
0 & 0 & Z_{\dot{w}_{f}} & 0 & 0 & 0 \\
0 & 0 & 0 & 0 & 0 & 0 \\
0 & 0 & 0 & 0 & M_{\dot{q}_{f}} & 0 \\
0 & 0 & 0 & 0 & 0 & 0
\end{array}\right]
$$

where the above matrix represents the added mass in the foil frame, as defined in Section 3.1.

Newman [14] gives the theoretical expressions for the added mass of a flat plate 
Table 4.2: Foil Added Mass Coefficients

\begin{tabular}{crll}
\hline \hline Parameter & Value & Units & Description \\
\hline$Z_{\dot{w}_{f}}$ & -186 & $\mathrm{~kg}$ & vertical added mass (foil frame) \\
$M_{\dot{q}_{f}}$ & -111 & $\mathrm{~kg} \cdot \mathrm{m}^{2}$ & pitch added mass (foil frame) \\
\hline \hline
\end{tabular}

with lateral extent $a$ :

$$
\begin{aligned}
& m_{11}=0 \\
& m_{22}=\pi \rho a^{2} \\
& m_{66}=\frac{1}{8} \pi \rho a^{4} .
\end{aligned}
$$

Approximating each foil pair as a series of flat strips aligned with the foil chords, the expressions for the added mass coefficients in (4.12) are:

$$
\begin{aligned}
Z_{\dot{w}_{f}} & =\frac{1}{4} \pi \rho \int_{x_{f}} L_{f}(x)^{2} d x \\
M_{\dot{q}_{f}} & =\frac{1}{4} \pi \rho \int_{x_{f}} x^{2} L_{f}(x)^{2} d x
\end{aligned}
$$

where $L_{f}(x)$ denotes the chord-wise cross-sectional length as a function of distance $x_{f}$ along the foil axis.

The resultant numerical values for the foil added mass coefficients are given in Table 4.2

\subsubsection{Combined Added Mass}

The added mass matrix of the entire vehicle, composed of the body and two pairs of foils, can be derived by considering the kinetic energy of the surrounding fluid:

$$
T=\frac{1}{2} \nu^{T} \boldsymbol{M}_{A}\left(\phi_{f}\right) \boldsymbol{\nu}
$$

Here $\boldsymbol{M}_{A}$ represents added mass matrix of the complete vehicle. As indicated, it is dependent on foil angle. It would violate conservation of energy to employ a nonconstant added mass matrix dependent on foil angle without increasing the order of the model. Therefore, $M_{A}\left(\phi_{f}\right)$ above should be interpreted as an expression for the constant added mass matrix of the complete vehicle for arbitrary, but fixed foil angles. 
It can be shown that the expression of $\boldsymbol{M}_{A}\left(\phi_{f}\right)$ for arbitrary foil angle is given by:

$$
\boldsymbol{M}_{A}=\boldsymbol{M}_{A_{b}}+\boldsymbol{U}_{f f}^{T} \boldsymbol{M}_{A_{f f}} \boldsymbol{U}_{f f}+\boldsymbol{U}_{a f}^{T} \boldsymbol{M}_{A_{a f}} \boldsymbol{U}_{a f}
$$

where the transformation matrix $U$ is given by:

$$
\boldsymbol{U}\left(\phi_{f}\right)=\left[\begin{array}{cc}
\boldsymbol{R}_{f} & 0_{3 \times 3} \\
0_{3 \times 3} & \boldsymbol{R}_{f}
\end{array}\right]^{-1}\left[\begin{array}{cc}
I_{3 \times 3} & -\boldsymbol{S}\left({ }^{\boldsymbol{b}} \boldsymbol{r}_{f_{0}}\right) \\
0_{3 \times 3} & I_{3 \times 3}
\end{array}\right]
$$

where $\boldsymbol{R}_{f}$ denotes the rotation matrix between the foil and body frames as defined in equation (3.6), and ${ }^{\boldsymbol{b}} r_{f_{0}}=\left[a_{f}, 0,0\right]^{T}$ denotes the vector from the origin of the body frame to the origin of each foil frame. The derivation (4.17) is given in Appendix A.

For nominally horizontal flight the foils will be nominally flat $\left(\phi_{f}=0\right)$ and the combined added mass matrix reduces to:

$$
M_{A}=\left[\begin{array}{cccccc}
X_{\dot{u}} & 0 & 0 & 0 & 0 & 0 \\
0 & Y_{\dot{v}} & 0 & 0 & 0 & N_{\dot{v}} \\
0 & 0 & Z_{\dot{w}} & 0 & M_{\dot{w}} & 0 \\
0 & 0 & 0 & K_{\dot{p}} & 0 & 0 \\
0 & 0 & M_{\dot{w}} & 0 & M_{\dot{q}} & 0 \\
0 & N_{\dot{v}} & 0 & 0 & 0 & N_{\dot{r}}
\end{array}\right] .
$$

where the individual terms of $\boldsymbol{M}_{A}$ are:

$$
\begin{aligned}
X_{\dot{u}} & =X_{\dot{u}_{b}} \\
Y_{\dot{v}} & =Y_{\dot{v}_{b}} \\
Z_{\dot{w}} & =Z_{\dot{w}_{b}}+Z_{\dot{w}_{f}} \\
K_{\dot{p}} & =K_{\dot{p}_{b}}+M_{\dot{q}_{f}} \\
M_{\dot{q}} & =M_{\dot{q}_{b}}+\left(a_{f f}^{2}+a_{a f}^{2}\right) Z_{\dot{w}_{f}} \\
N_{\dot{r}} & =N_{\dot{r}_{b}} \\
M_{\dot{w}} & =M_{\dot{w}_{b}}-\left(a_{f f}+a_{a f}\right) Z_{\dot{w}_{f}} \\
N_{\dot{v}} & =N_{\dot{v}_{b}}
\end{aligned}
$$

Numerical values for these coefficients are given in Table 4.3.

The total forces and moments due to added mass incident on the complete vehicle 
Table 4.3: Combined Added Mass Coefficients $\left(\phi_{f}=0\right)$

\begin{tabular}{crll}
\hline \hline Parameter & Value & Units & Description \\
\hline$X_{\dot{u}}$ & -366 & $\mathrm{~kg}$ & combined axial added mass \\
$Y_{\dot{v}}$ & -4960 & $\mathrm{~kg}$ & combined lateral added mass \\
$Z_{\dot{w}}$ & -1520 & $\mathrm{~kg}$ & combined vertical added mass \\
$K_{\dot{p}}$ & -264 & $\mathrm{kgm}^{2}$ & combined roll added mass \\
$M_{\dot{q}}$ & -1020 & $\mathrm{kgm}^{2}$ & combined pitch added mass \\
$N_{\dot{r}}$ & -2210 & $\mathrm{kgm}^{2}$ & combined yaw added mass \\
$M_{\dot{w}}$ & -247 & $\mathrm{kgm}$ & combined added mass cross term \\
$N_{\dot{v}}$ & 815 & $\mathrm{kgm}$ & combined added mass cross term \\
\hline \hline
\end{tabular}

can be expressed in matrix form [5] as:

$$
-M_{A} \dot{\nu}-C_{A}(\nu) \nu=\tau_{A}
$$

where the expression for $C_{A}$ is

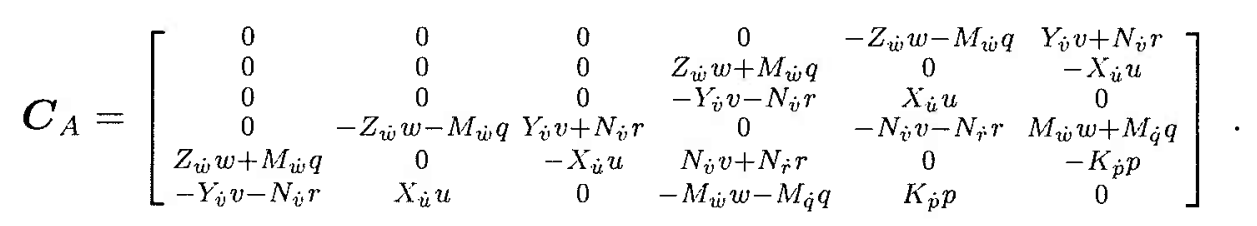

The terms $\left(X_{\dot{u}}-Z_{\dot{w}}\right) u w$ and $-\left(X_{\dot{u}}-Y_{\dot{v}}\right) u v$ in the expanded expression for $\tau_{A}$ are known as Munk moments [21] in the longitudinal and lateral planes respectively, and express the pure moment that acts on a body at an angle of attack (or side slip) in potential flow.

\subsection{Strip-Theory Lift and Drag}

The flow around the foils and roots will be predominantly two-dimensional at small angles of attack. The streamlined, foil-like shape of the main hull suggests that the flow around it will also be somewhat two-dimensional, although to a lesser extent.

Relying heavily on strip-theory, in this section we determine the quadratic lift and crossflow drag forces incident on the vehicle. In this approach, the 2D lift and drag forces and moments of individual hull and foil cross-sections are integrated to yield total hydrodynamic forces and moments. The longitudinal crossflow drag of the hull, lateral lift of the hull, and the lift and drag of the foils are all treated in this unified context. 
The advantage of this approach is that different fluid velocities across different portions of the vehicle are accounted for. The disadvantages of this approach are its reliance on strip theory and that derived models contain integrals, slowing numerical simulation.

The relevant aspect ratios of the hull, roots and control foils are given in Table 4.4. Listed ratios for the foils are defined as average span divided by average chord $\bar{b} / \bar{c}$. For the roots this value is multiplied by 2 to yield the effective aspect ratio due to the presence of the hull. Our reliance on strip-theory to compute the lateral lift forces of the hull is justifiably questionable given the relatively small length to height ratio $(\mathrm{L} / \mathrm{H})$ of the main hull. Where possible, we apply corrections for the three-

Table 4.4: Geometry Relevant to Strip-Theory

\begin{tabular}{cccl}
\hline \hline Ratio & Value & Expression & Description \\
\hline$A R_{b_{x}}$ & 0.7 & $H / L$ & hull height to length ratio \\
$A R_{b_{z}}$ & 4.3 & $L / W$ & hull length to width ratio \\
$A R_{f}$ & 1.5 & $\bar{b}_{f} / \bar{c}_{f}$ & control foil aspect ratio \\
$A R_{f} r$ & 0.8 & $\bar{b}_{f r} / \bar{c}_{f r}$ & effective forward root aspect ratio \\
$A R_{a} r$ & 1.3 & $\bar{b}_{a r} / \bar{c}_{a r}$ & effective aft root aspect ratio \\
\hline \hline
\end{tabular}

dimensional nature of the actual flow by the appropriate modification of leading coefficients.

The longitudinal lift of the hull, for which the relevant aspect ratio is too small to apply this technique, is treated separately in Section 4.4.

\subsubsection{Basic Results from Dimensional Analysis}

From a basic dimensional analysis of lift and drag forces on a body moving through a fluid (see for example Newman [14]), the lift and drag forces can be written:

$$
\begin{aligned}
& L=\frac{1}{2} \rho C_{L}(\alpha, R n) A|V| V \\
& D=\frac{1}{2} \rho C_{D}(\alpha, R n) A|V| V .
\end{aligned}
$$

Here, $C_{L}(\alpha, R n)$ denotes a non-dimensional lift coefficient and $C_{D}(\alpha, R n)$ a nondimensional drag coefficient. Both are dependent on the angle of attack of the body $\alpha$ and on the Reynolds number $R n_{L}=\frac{\rho V L}{\mu}$ of the flow. The constant $A$ denotes the characteristic area of the body, and $V$ a characteristic velocity. 
Table 4.5: 2D Section Lift and Drag Leading Coefficients

\begin{tabular}{ccl}
\hline \hline Coefficient & Value & Description \\
\hline$K_{L_{b x y}}$ & 1.1 & hull lateral lift \\
$K_{D_{b x y}}$ & 0.0 & hull lateral induced drag \\
$K_{D o_{b x y}}\left(z_{b}\right)$ & - & hull parasitic drag (eq. (4.26)) \\
$K_{D o_{b y z}}\left(x_{b}\right)$ & - & hull crossflow drag (eq. (4.27)) \\
$K_{L_{f f, a f}}$ & 1.69 & control foil lift \\
$K_{D_{f f, a f}}$ & 1.9 & control foil drag at $\alpha=90^{\circ}$ \\
$K_{D o_{f f, a f}}\left(x_{f}\right)$ & - & control foil parasitic drag \\
$K_{L_{f r}}$ & 1.00 & forw. root lift \\
$K_{D f r}$ & 1.25 & forw. drag at $\alpha=90^{\circ}$ \\
$K_{D o_{f r}}\left(x_{r}\right)$ & - & forw. root parasitic drag \\
$K_{L_{a r}}$ & 1.51 & aft root lift \\
$K_{D_{a r}}$ & 1.25 & aft root drag at $\alpha=90^{\circ}$ \\
$K_{D o_{a r}}\left(x_{r}\right)$ & - & aft root parasitic drag \\
\hline \hline
\end{tabular}

\subsubsection{Lift and Drag Coefficient Approximation}

The lift and drag coefficients $C_{L}$ and $C_{D}$ of the previous section are in general complex functions of their arguments; however, simplified analytic approximations are available for a number of practical situations, and a wealth of experimental data exists for typical aerodynamic shapes (e.g. $[8,9,11]$, and references therein).

The foil-like shapes considered in this section create lift at non-zero angle of attack $\alpha$ and are subject to drag induced by the production of lift ("induced drag"), due to their finite aspect ratio and the production of vortices. In addition to induced drag, a typically smaller component of the total drag, "parasitic drag" is due to viscous boundary layer losses and roughly invariant over small angles of attack.

As indicated in equations (4.22) and (4.23), the lift and drag coefficients are also functions $R n$. In terms of modeling accuracy, lift and drag forces are most important at cruising speeds. For vehicle speeds between $0.75 \mathrm{~m} / \mathrm{s}$ and $2.0 \mathrm{~m} / \mathrm{s}, R e_{L}$ varies between $1.3 \times 10^{6}$ and $3.6 \times 10^{6}$ for the hull; well into the turbulent flow regime. Ignoring hull interaction, over this speed range the Reynolds numbers of the control foils and roots are smaller: on the order of $0.3 \times 10^{6} \leq R n_{\bar{c}} \leq 0.8 \times 10^{6}$ referenced to average foil chord. These values fall in the transitional region between laminar and turbulent flow. Throughout the following analysis we assume fully developed turbulent flow and ignore $R n$-dependence.

Leading coefficients relevant to lift and drag forces on the Sentry vehicle are derived in the following three sections and summarized in Table 4.5 


\section{Hull Lateral Lift and Drag Coefficients}

For the particular case of the Sentry AUV, we will consider the lateral lift and drag coefficients of the main hull separately from the foils and stationary foil roots. This treatment is motivated by the observation that the vehicle has no means of thrusting laterally and as a result the main hull will experience only small angles of side slip when moving forward at speeds sufficient to produce significant lateral body lift. Simple, accurate models for lift coefficients are available for low aspect ratio wings limited to small angle of attack, and for this reason we will determine the lateral lift and drag coefficients of the main hull from a linear approximation of the form

$$
C_{L}=K_{L} \alpha ; \quad C_{D}=K_{D_{o}}
$$

where $K_{L}$ and $K_{D_{o}}$ are constant approximations of the slope of the lift and drag coefficient curves about $\alpha=0$. Note that we approximate the drag coefficient of the body as independent of angle of attack.

Treating the main hull roughly as a small aspect ratio wing, the lift coefficient is theoretically [9], pg. 17-2 (5):

$$
K_{L_{b: y}}=\frac{\pi}{2} A R_{b_{x}}
$$

The excessive thickness of the hull compared to flat plate implies that (4.25) represents an overestimate.

The 2D of hull cross-sections varies with thickness to chord ratio $t / c$. Employing Hoerner's expression for the 2D drag of an airfoil section ([8], pg. 6-6 (6)),

$$
K_{D o_{b x y}}(z)=2 C_{f}\left(1+2 \frac{W_{b}(z)}{L_{b}(z)}+60 \frac{W_{b}(z)^{4}}{L_{b}(z)}\right.
$$

where $C_{f}$ is the skin friction coefficient. Schoenherr's line predicts $C_{f}=0.0039$ for fully turbulent flow corresponding to $R n=2.1 \times 10^{6}$.

\section{Hull Longitudinal Crossflow Drag Coefficient}

The 2D longitudinal crossflow drag coefficient of an elliptical cross section is given by Hoerner [8], pg. 3-11 (21):

$$
K_{D o_{b y z}}(x)=C_{f_{t u r b}}\left(4+2 \frac{H_{b}(x)}{W_{b}(x)}+120\left(\frac{W_{b}(x)}{H_{b}(x)}\right)^{2}\right) .
$$

We again assume fully turbulent flow and choose $C_{f_{t u r b}}=0.0039$. 


\section{Control Foil and Root Coefficients}

The control foils and particularly the stationary foil roots may be subjected to relatively large angles of attack, and potentially even complete flow reversal depending on the operating mode of the vehicle. This necessitates the use of a broader model for the lift and drag coefficients. For this latter case, Hoerner [9] suggests the approximation

$$
\begin{aligned}
& C_{L}=K_{L} \sin \alpha \cos \alpha \\
& C_{D}=K_{D} \sin ^{2} \alpha .
\end{aligned}
$$

For the leading coefficients $K_{L}$ and $K_{D}$, Hoerner suggests values between 1.8 and 2.0.

Equations (4.28) and (4.29) fail to capture the complex lift and drag behavior of foils near stall. This shortcoming is partially mitigated by the hydrodynamic characteristics of small aspect ratio (AR) foils such as those on Sentry which stall at a much higher angle of attack than slender foils [4]; however, small AR foils exhibit a steeper lift curve at high angles of attack $([9],[4])$ in disagreement the decreasing slope predicted in (4.28).

It is impossible to account for these structural inaccuracies by appropriate choice of the leading coefficients in (4.28) and (4.29). Instead, we choose the leading coefficients such that lift is predicted accurately at low angles of attack, and drag is predicted accurately at high angles of attack. This choice is motivated by the author's experience with the physical model (Appendix B) which indicates the vehicle roughly follows a trajectory tangent to the control foils when operating at speeds significant enough to warrant consideration of quadratic lift and drag forces. To adequately model such behavior we require an accurate model of foil lift for small to medium angles of attack, and a model that accurately captures the increase in drag, particularly from the foil roots, for large vertical velocity. Also, foil and root drag at $\alpha \approx 90^{\circ}$ is important for pitch damping at low forward speed.

Empirical lift coefficients for low aspect ratio foils of similar shape, as determined by Whicker and Fehlner (1958) are summarized in [11]. Linearly interpolating their results for the aspect ratios of the control foils and roots provides the numerical values for $K_{L_{f f, a f}}$ and $K_{L_{f r, a r}}$ listed in Table 4.5 .

We will approximate the profile drag of the control foils at $\alpha=90^{\circ}$ as that of a rectangular plate of identical area. Hoerner [8], pg. 4-23 (17), suggests a numerical value of 1.9 for a plate in turbulent, free-stream flow. We will use this value for $K_{D_{f f, a f}}$.

The roots interact with the hull to a greater extent than the foils. Hoerner [8], 


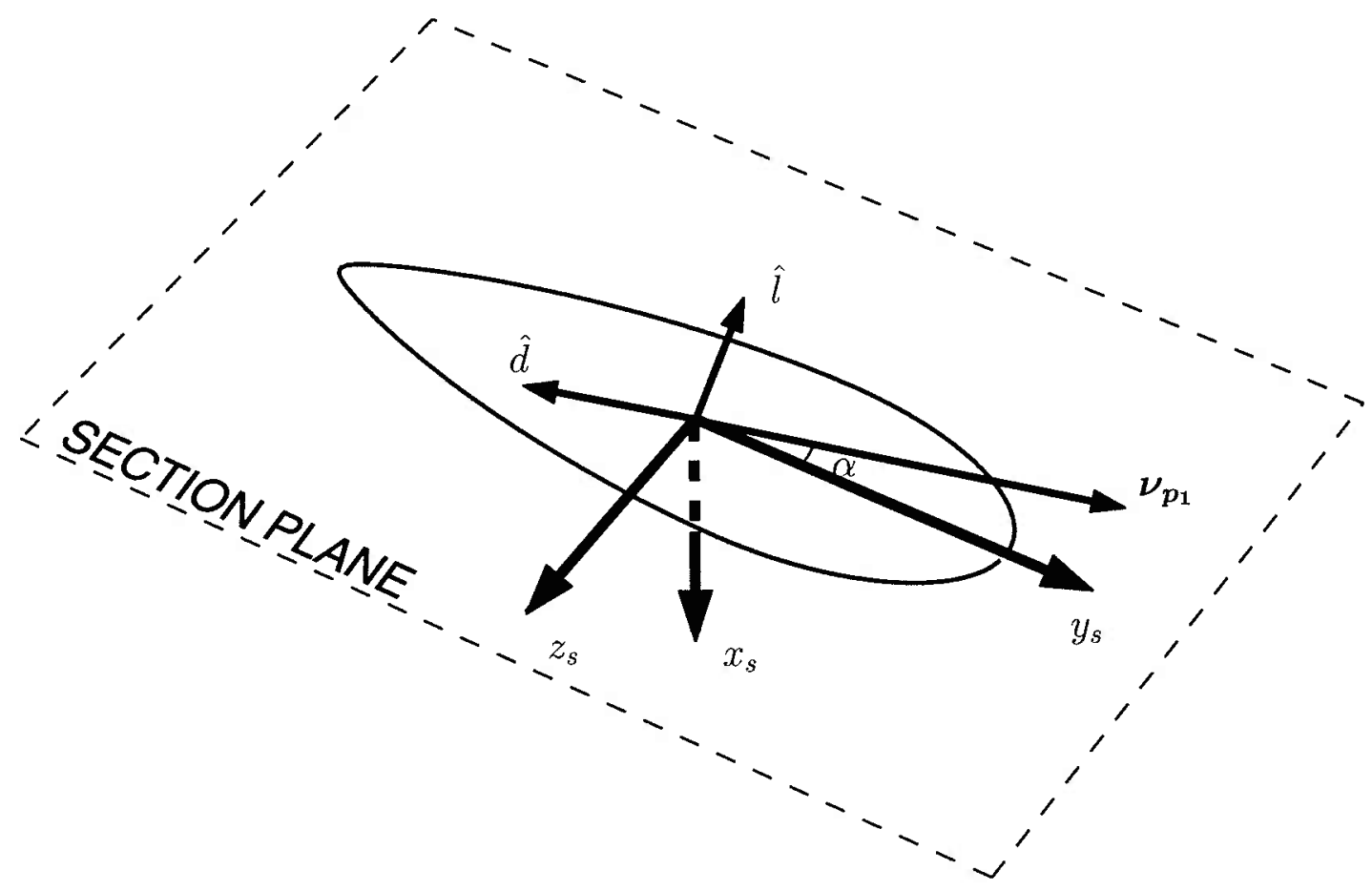

Figure 4-1: Aerodynamic Section Definition

pg. 8-3, provides an estimate of $C_{D_{\mathbf{\bullet}}}=1.25$ for the coefficient of drag of a plate protruding from a streamlined body.

The parasitic component of drag for both the control foils and roots is small compared to the drag of the hull and we ignore it; however, depending on design the thruster nacelles may have a significant effect on control foil lift and drag. We have not estimated this additional source of drag.

\subsubsection{Differential Lift and Drag of an Aerodynamic Section}

Assuming strip-theory applies, we can determine the lift and drag forces of any aerodynamically shaped body if we know its $2 \mathrm{D}$ lift and drag characteristics and the geometry relating it to the motion an arbitrary body-fixed frame. In this section we derive a general expression for the differential lift and drag of a two-dimensional aerodynamic section oriented into the flow, but of arbitrary orientation relative to a body-fixed reference frame. This technique will allow us to consider the foils and the body in a unified framework.

We define a local coordinate frame for a body composed of aerodynamic twodimensional sections as shown in Figure 4-1. In our convention, the $x_{s}$-direction is 
perpendicular to plane of each cross-section and that the $y_{s}$-direction is parallel to the nominal direction of motion.

In vector form, the quadratic lift and drag equations, (4.22) and (4.23), can be written:

$$
\begin{aligned}
{ }^{b} d \boldsymbol{L}_{s} & =\frac{1}{2} \rho C_{L} b\left(x_{s}\right)\left({ }^{b} \boldsymbol{\nu}_{\boldsymbol{p}_{\mathbf{1}}}\right)^{T}{ }^{b} \boldsymbol{\nu}_{\boldsymbol{p}_{1}} d x_{s}{ }^{b} \hat{\boldsymbol{l}} \\
{ }^{b} d \boldsymbol{D}_{\boldsymbol{s}} & =\frac{1}{2} \rho C_{D} b\left(x_{s}\right)\left({ }^{b} \boldsymbol{\nu}_{\boldsymbol{p}_{\mathbf{1}}}\right)^{T}{ }^{T} \boldsymbol{\nu}_{\boldsymbol{p}_{1}} d x_{s}{ }^{b} \hat{\boldsymbol{d}} .
\end{aligned}
$$

where ${ }^{b} \boldsymbol{\nu}_{\boldsymbol{p}_{1}}$ indicates in-plane section velocity, $\hat{l}$ and $\hat{d}$ are the unit vectors parallel and perpendicular to the flow respectively as shown in Figure 4-1. The quantity $b\left(x_{s}\right)$ denotes the characteristic width of the section.

The lift and drag unit vectors can be expressed in terms of the in-plane section velocity ${ }^{b} \nu_{p_{1}}$ as follows:

$$
{ }^{b} \hat{d}=-\frac{{ }^{b} \boldsymbol{\nu}_{p_{1}}}{\left\|{ }^{b} \boldsymbol{\nu}_{p_{1}}\right\|} .
$$

Recognizing that

$$
s \hat{l}={ }^{s} \hat{i} \times{ }^{s} \hat{d}
$$

the unit lift vector ${ }^{s} \hat{l}$ becomes:

$$
\begin{aligned}
{ }^{b} \hat{l} & =\left(\boldsymbol{R}_{s}{ }^{s} \hat{i}\right) \times{ }^{b} \hat{d} \\
{ }^{b} \hat{l} & =\boldsymbol{S}\left(\boldsymbol{R}_{s}{ }^{s} \hat{i}\right){ }^{b} \hat{d} \\
{ }^{b} \hat{l} & =-\boldsymbol{S}\left(\boldsymbol{R}_{s}{ }^{s} \hat{i}\right) \frac{{ }^{b} \boldsymbol{\nu}_{p_{1}}}{\left\|{ }^{b} \boldsymbol{\nu}_{p_{1}}\right\|}
\end{aligned}
$$

where $\boldsymbol{R}_{\boldsymbol{s}}$ denotes the rotation matrix between section and body frames such that for any vector $\boldsymbol{c},{ }^{b} \boldsymbol{c}=\boldsymbol{R}_{\boldsymbol{s}}{ }^{s} \boldsymbol{c}$. In the above we have used Fossen's [5] notation $\boldsymbol{S}(\boldsymbol{c})$ to denote a skew symmetric matrix such that $\boldsymbol{c} \times \boldsymbol{a} \equiv \boldsymbol{S}(\boldsymbol{c}) \boldsymbol{a}$.

Using the coordinate frame convention shown in Figure 4-1, we can derive an expression for ${ }^{b} \nu_{p_{1}}$ in terms of the body-fixed velocity ${ }^{b} \boldsymbol{\nu}_{\boldsymbol{b}}$ :

$$
\begin{aligned}
& { }^{s} \nu_{p_{1}}=P^{s} \nu_{s_{1}} \\
& { }^{b} \nu_{s_{1}}=R_{s}{ }^{s} \nu_{s_{1}} \\
& { }^{b} \nu_{s_{1}}={ }^{b} \nu_{b_{1}}-S\left({ }^{b} r_{s}\right)^{b} \nu_{b_{2}} \\
& { }^{b} \nu_{p_{1}}=R_{s}{ }^{s} \nu_{p_{1}}
\end{aligned}
$$


where $\boldsymbol{P}$ is a rank-deficient projection matrix defined as:

$$
\boldsymbol{P}=\left[\begin{array}{lll}
0 & 0 & 0 \\
0 & 1 & 0 \\
0 & 0 & 1
\end{array}\right]
$$

$P$ projects the section velocity ${ }^{s} \nu_{s_{1}}$ onto the section plane. Note from the geometry that

$$
{ }^{b} \boldsymbol{r}_{\boldsymbol{s}}\left(x_{s}\right)={ }^{b} \boldsymbol{r}_{\boldsymbol{o}}+x_{s} \boldsymbol{R}_{\boldsymbol{s}}{ }^{s} \hat{i}_{s}
$$

Using the above expressions,

$$
\begin{aligned}
{ }^{b} \boldsymbol{\nu}_{p_{1}} & =\boldsymbol{R}_{\boldsymbol{s}} \boldsymbol{P} \boldsymbol{R}_{\boldsymbol{s}}{ }^{-1}\left[I_{3 \times 3}-\boldsymbol{S}\left({ }^{b} \boldsymbol{r}_{\boldsymbol{s}}\left(x_{s}\right)\right)\right]{ }^{b} \boldsymbol{\nu}_{\boldsymbol{b}} \\
& =\boldsymbol{R}_{\boldsymbol{s}} \boldsymbol{G}_{\boldsymbol{s}}{ }^{b} \boldsymbol{\nu}_{\boldsymbol{b}}
\end{aligned}
$$

where we have introduced the transformation matrix $\boldsymbol{G}_{\boldsymbol{s}}$ to simplify notation.

Substituting (4.40) and the expressions for the lift and drag unit vectors (4.33) and (4.32) into (4.30) gives an expression for differential lift and drag in terms of the body-fixed velocity ${ }^{b} \boldsymbol{\nu}_{\boldsymbol{b}}$, and lift and drag coefficients $C_{L}$ and $C_{D}$ :

$$
\begin{aligned}
& { }^{b} d \boldsymbol{L}_{\boldsymbol{s}}=-\frac{1}{2} \rho C_{L} b\left(x_{s}\right)\left(\boldsymbol{R}_{\boldsymbol{s}} \boldsymbol{G}_{\boldsymbol{s}}{ }^{b} \boldsymbol{\nu}_{\boldsymbol{b}}\right)^{T} \boldsymbol{R}_{\boldsymbol{s}} \boldsymbol{G}_{\boldsymbol{s}}{ }^{b} \boldsymbol{\nu}_{\boldsymbol{b}} \boldsymbol{S}\left(\boldsymbol{R}_{\boldsymbol{s}}{ }^{s} \hat{i}\right) \frac{\boldsymbol{R}_{\boldsymbol{s}} \boldsymbol{G}_{\boldsymbol{s}}{ }^{b} \boldsymbol{\nu}_{\boldsymbol{b}}}{\left\|\boldsymbol{R}_{\boldsymbol{s}} \boldsymbol{G}_{\boldsymbol{s}}{ }^{b} \boldsymbol{\nu}_{\boldsymbol{b}}\right\|} d x_{s} \\
& { }^{b} d \boldsymbol{D}_{\boldsymbol{s}}=-\frac{1}{2} \rho C_{D} b\left(x_{s}\right)\left(\boldsymbol{R}_{\boldsymbol{s}} \boldsymbol{G}_{\boldsymbol{s}}{ }^{b} \boldsymbol{\nu}_{\boldsymbol{b}}\right)^{T} \boldsymbol{R}_{\boldsymbol{s}} \boldsymbol{G}_{\boldsymbol{s}}{ }^{b} \boldsymbol{\nu}_{\boldsymbol{b}} \frac{\boldsymbol{R}_{\boldsymbol{s}} \boldsymbol{G}_{\boldsymbol{s}}{ }^{b} \boldsymbol{\nu}_{\boldsymbol{b}}}{\left\|\boldsymbol{R}_{\boldsymbol{s}} \boldsymbol{G}_{\boldsymbol{s}}{ }^{b} \boldsymbol{\nu}_{\boldsymbol{b}}\right\|} d x_{s}
\end{aligned}
$$

\section{Application to Sentry}

The leading coefficients $C_{L}$ and $C_{D}$ are still arbitrary at this point. To determine the differential lift and drag of a lateral section of the main hull, the constant leading coefficients given in Section 4.2.2 need only be substituted for $C_{L}$ and $C_{D}$ in equation 4.41 and $\boldsymbol{R}_{s}$ set such that the vehicle axes translate accordingly into our convention for the section frame.

For the control foils and roots, the approximations for $C_{L}$ and $C_{D}$ given in Section 4.2 .2 also enable 4.41 to be written entirely in terms of the body-fixed velocity ${ }^{b} \nu_{\boldsymbol{b}}$. The computation is given below.

From Figure 4-1, the angle of attack $\alpha$ can be written in terms of the dot product

$$
\left\|{ }^{s} \nu_{p_{1}}\right\| \cos \alpha={ }^{s} \nu_{p_{1}} \cdot{ }^{s} \hat{j}_{s}
$$


or equivalently,

$$
\left\|{ }^{s} \boldsymbol{\nu}_{\boldsymbol{p}_{\mathbf{1}}}\right\| \sin \alpha={ }^{s} \boldsymbol{\nu}_{\boldsymbol{p}_{\mathbf{1}}}{ }^{s} \hat{k}_{s} .
$$

Substituting these expressions into (4.28) and (4.29) and ignoring dynamic Re-dependence,

$$
\begin{aligned}
C_{L} & =K_{L} \frac{\left({ }^{s} \boldsymbol{\nu}_{p_{\mathbf{1}}} \cdot s \hat{k}_{s}\right)\left({ }^{s} \boldsymbol{\nu}_{\boldsymbol{p}_{\mathbf{1}}} \cdot s \hat{j}_{s}\right)}{\left\|{ }^{s} \boldsymbol{\nu}_{\boldsymbol{p}_{1}}\right\|^{2}} \\
C_{D} & =K_{D_{o}}+K_{D} \frac{\left({ }^{s} \boldsymbol{\nu}_{\boldsymbol{p}_{\mathbf{1}}} \cdot{ }^{s} \hat{k}_{s}\right)\left({ }^{s} \boldsymbol{\nu}_{\boldsymbol{p}_{\mathbf{1}}} \cdot s \hat{k}_{s}\right)}{\left\|{ }^{s} \boldsymbol{\nu}_{p_{1}}\right\|^{2}} .
\end{aligned}
$$

Substituting the result of (4.34) for ${ }^{s} \boldsymbol{\nu}_{p_{1}}$, and rearranging the dot products gives

$$
\begin{aligned}
C_{L} & =K_{L} \frac{\left(\boldsymbol{G}_{\boldsymbol{s}}{ }^{b} \boldsymbol{\nu}_{\boldsymbol{b}}\right)^{T s} \hat{k}_{s}{ }^{s} \hat{j}_{s}^{T}\left(\boldsymbol{G}_{\boldsymbol{s}}{ }^{b} \boldsymbol{\nu}_{\boldsymbol{b}}\right)}{\left(\boldsymbol{G}_{\boldsymbol{s}}{ }^{b} \boldsymbol{\nu}_{\boldsymbol{b}}\right)^{T} \boldsymbol{G}_{\boldsymbol{s}}{ }^{b} \boldsymbol{\nu}_{\boldsymbol{b}}} \\
C_{D} & =K_{D_{o}}+K_{D} \frac{\left(\boldsymbol{G}_{\boldsymbol{s}}{ }^{b} \boldsymbol{\nu}_{\boldsymbol{b}}\right)^{T s} \hat{k}_{s}{ }^{s} \hat{k}_{s}^{T}\left(\boldsymbol{G}_{\boldsymbol{s}}{ }^{b} \boldsymbol{\nu}_{\boldsymbol{b}}\right)}{\left(\boldsymbol{G}_{\boldsymbol{s}}{ }^{b} \boldsymbol{\nu}_{\boldsymbol{b}}\right)^{T} \boldsymbol{G}_{\boldsymbol{s}}{ }^{b} \boldsymbol{\nu}_{\boldsymbol{b}}}
\end{aligned}
$$

Finally, substituting (4.46) and (4.47) into (4.41) and simplifying gives

$$
\begin{array}{r}
d \boldsymbol{L}_{\boldsymbol{s}}=-K_{L} b\left(x_{s}\right)\left(\left(\frac{\left(\boldsymbol{G}_{\boldsymbol{s}}{ }^{b} \boldsymbol{\nu}_{\boldsymbol{b}}\right)^{T s} \hat{k}_{s}{ }^{s} \hat{j}_{s}^{T}\left(\boldsymbol{G}_{\boldsymbol{s}} \boldsymbol{\nu}_{\boldsymbol{b}}\right)}{\left\|\boldsymbol{G}_{\boldsymbol{s}}{ }^{b} \boldsymbol{\nu}_{\boldsymbol{b}}\right\|}\right) \boldsymbol{S}\left(\boldsymbol{R}_{\boldsymbol{s}}{ }^{s} \hat{i}_{s}\right) \boldsymbol{R}_{\boldsymbol{s}} \boldsymbol{G}_{\boldsymbol{s}} d x_{s}\right){ }^{b} \boldsymbol{\nu}_{\boldsymbol{b}} \\
d \boldsymbol{D}_{\boldsymbol{s}}=-K_{D_{o}} b\left(x_{s}\right)\left(\left\|\boldsymbol{G}^{b} \boldsymbol{\nu}_{\boldsymbol{b}}\right\| \boldsymbol{R}_{\boldsymbol{s}} \boldsymbol{G}_{\boldsymbol{s}} d x_{s}\right)^{b} \boldsymbol{\nu}_{\boldsymbol{b}} \\
-K_{D} b\left(x_{s}\right)\left(\left(\frac{\left(\boldsymbol{G}_{\boldsymbol{s}}{ }^{b} \boldsymbol{\nu}_{\boldsymbol{b}}\right)^{T s} \hat{k}_{s}{ }^{s} \hat{k}_{s}^{T}\left(\boldsymbol{G}_{\boldsymbol{s}}{ }^{b} \boldsymbol{\nu}_{\boldsymbol{b}}\right)}{\left\|\boldsymbol{G}_{\boldsymbol{s}}{ }^{b} \boldsymbol{\nu}_{\boldsymbol{b}}\right\|}\right) \boldsymbol{R}_{\boldsymbol{s}} \boldsymbol{G}_{\boldsymbol{s}} d x_{s}\right){ }^{b} \boldsymbol{\nu}_{\boldsymbol{b}}
\end{array}
$$

Note that these expressions are singular for ${ }^{b} \boldsymbol{\nu}_{b}=0$. Physically, the angle of attack $\alpha$ is undefined at zero velocity.

\subsection{Quadratic Hydrodynamic Moments}

Lift and drag induced pressure forces created by fluid flowing around a lifting body can be taken to act at a point called the center of pressure (CP). Thus for body-fixed reference frames whose origins do not coincide with the CP, lift and drag forces exert a moment about the frame's origin. In general, the center of pressure moves somewhat as angle of attack is increased; however, we will assume a fixed CP. 


\begin{tabular}{ccl} 
Table 4.6: Section CP Location (\% Chord) \\
\hline \hline Symbol & $\%$ Chord & Section \\
\hline$\% c_{b}$ & $0.25^{*}$ & hull ${ }^{*}$ See below. \\
$\% c_{f}$ & 0.18 & control foils \\
$\% c_{r}$ & 0.18 & roots \\
\hline \hline
\end{tabular}

\subsubsection{Center of Pressure Locations}

We need only determine locations of centers of pressure for the 2D sections comprising a body to compute sectional hydrodynamic moments. These locations are given as a percentage of chord in Table 4.6 for the lateral hull, control foil, and root sections.

\section{Hull Section CP Locations}

In this section we have treated the lateral hydrodynamics of the hull as that of a wing. The fullness of the hull and its small aspect ratio suggest that in reality it will behave to some degree like a conventional rotationally symmetric hull in that a suction force will be created by vortices shed from the aft portion $[9,21]$. Furthermore, we have already computed a lateral Munk moment associated with the hull's lateral hydrodynamics in Section 4.1. The pure couple associated with a Munk moment alters the location of the hull's CP from that of a 2D wing which theoretically does not generate a pure couple.

We will assume 2D sectional lift and drag forces act at the theoretical $\frac{1}{4}$-chord center of pressure for a thin foil. As we lack an estimate of the suction force mentioned above, the lateral destabilizing moment of the hull will be overestimated.

\section{Control Foil and Root Section CP Locations}

The values for $\% c_{f}$ and $\% c_{r}$ in Table 4.6 are derived from data for low aspect ratio control surfaces presented in [11].

\subsubsection{Differential Moment of an Aerodynamic Section}

Referring to Figure 4-1, the in-plane differential moment generated by a differential force $d \boldsymbol{F}_{p}$ applied at the CP of a 2D section about the an arbitrary body-fixed origin is given by

$$
d \boldsymbol{M}_{\boldsymbol{p}}=\boldsymbol{r}_{\boldsymbol{C P}} \times\left(d \boldsymbol{L}_{\boldsymbol{p}}+d \boldsymbol{D}_{\boldsymbol{p}}\right)
$$


where

$$
{ }^{b} \boldsymbol{r}_{\boldsymbol{C P}}={ }^{b} \boldsymbol{r}_{\boldsymbol{o}}+R_{s}\left(x_{s}{ }^{3} \hat{i}_{s}+{ }^{s} \boldsymbol{r}_{\boldsymbol{C P}}\right)
$$

The in-plane section moment arm $r_{C P_{s}}$ is given by

$$
{ }^{s} r_{C P_{s}}=\left[0, y_{C P_{s}}, 0\right]^{T}
$$

The sign and magnitude of $y_{C P_{s}}$ will change depending on the fluid direction over the section. Assuming that the CP is located at an approximately constant percentage of the chord away from the current leading edge regardless of direction of motion, and denoting this percentage by $(\% c)$,

$$
y_{C P_{s}}=\frac{1}{2}\left(L_{l e}+L_{t e}+\operatorname{sgn}\left(v_{s}\right)\left(L_{l e}-L_{t e}\right)\left(\frac{1-2(\% c)}{2}\right)\right) \text {. }
$$

The distance from the section origin to the leading edge $L_{l e}$ and to the trailing edge $L_{t e}$ are constant and defined relative to the nominal direction of motion. The inplane velocity $v_{s}$ can be expressed in terms of the body-fixed velocity ${ }^{b} \nu_{b}$ by the transformation

$$
v_{s}=s \hat{j}_{s}^{T s} \boldsymbol{\nu}_{\boldsymbol{p}_{\mathbf{1}}}={ }^{s} \hat{j}_{s}^{T} \boldsymbol{G}_{\boldsymbol{s}}{ }^{b} \boldsymbol{\nu}_{\boldsymbol{b}}
$$

\subsubsection{Combined Lift and Drag}

We are ultimately interested in the components of lift and drag forces in the principal directions of the vehicle being modeled. Projecting the quadratic differential lift and drag expressions of Section 4.2.3 and moment expressions of Section 4.3.2 onto each axis of the vehicle and then integrating gives the desired result. For example,

$$
\begin{aligned}
X & =\int_{x_{s}}{ }^{b} \hat{i}_{b}^{T}\left(d \boldsymbol{L}_{\boldsymbol{p}}+d \boldsymbol{D}_{\boldsymbol{p}}\right) d x \\
K & =\int_{x_{s}}{ }^{b} \hat{i}_{b}^{T}\left(d \boldsymbol{M}_{\boldsymbol{p}}\right) d x
\end{aligned}
$$

where the integrals in each expression are over the appropriate section geometry.

These expressions can be assembled into the convenient form of a matrix of damping terms derived from strip-theory. The trailing factor of ${ }^{b} \nu_{b}$ from the quadratic differential lift and drag expressions of the previous sections can be moved outside of the integrand in each expression to yield $1 \times 6$ vectors of integrals. These vectors can themselves be assembled into a positive definite $6 \times 6$ matrix of lift and damping 
terms:

$$
\boldsymbol{\tau}_{\text {s.t. }}=\boldsymbol{D}_{\text {s.t. }}{ }^{b} \boldsymbol{\nu}_{\boldsymbol{b}}=-\left[\begin{array}{c}
\int_{x_{s}} \boldsymbol{d}_{1}\left({ }^{b} \boldsymbol{\nu}_{\boldsymbol{b}}\right) d x \\
\vdots \\
\int_{x_{s}} \boldsymbol{d}_{6}\left({ }^{b} \boldsymbol{\nu}_{\boldsymbol{b}}\right) d x
\end{array}\right]^{b} \boldsymbol{\nu}_{\boldsymbol{b}}
$$

Here each integrand $\boldsymbol{d}_{i}\left({ }^{b} \boldsymbol{\nu}_{\boldsymbol{b}}\right)$ is a $1 \times 6$ vector of quadratic lift and drag terms that when multiplied by ${ }^{b} \boldsymbol{\nu}_{\boldsymbol{b}}$ yields the external forces acting on each axis of the vehicle. Note that $\boldsymbol{D}_{\boldsymbol{s . t}}$ is positive definite because it is composed purely of forces with non-zero components opposing the direction of motion.

\subsubsection{Expanded Expressions for Quadratic Lift and Drag}

For the Sentry AUV, each vector integrand $\boldsymbol{d}_{i}$ in 4.56 is a function of the quadratic lift and drag contributions from the hull, stationary roots, and foils. In matrix form, the lift and drag forces on the vehicle derived from strip-theory can be expressed component-wise as

$$
D_{s . t .}=D_{b x y}+D_{b y z}+D_{f f}\left(\phi_{f f}\right)+D_{a f}\left(\phi_{a f}\right)+D_{f r}+D_{a r}
$$

The subscript ${ }_{b} x y$ denotes hull lateral lift and drag. Subscript ${ }_{b} y z$ denotes longitudinal crossflow drag. Subscripts $f f$,af denote control foil lift and drag and subscripts fr,ar denote root lift and drag.

Explicit expressions for these matrices can be computed. The procedure for each component of the vehicle is the same. The geometric rotation matrix, $\boldsymbol{R}_{s}$ along with the geometric displacement vector ${ }^{b} \boldsymbol{r}_{s}$ are given by the geometry of the vehicle and are used to evaluate $\boldsymbol{G}_{s}$. The results are then substituted into the general lift, drag and moment expressions developed in Sections 4.2 .3 and 4.3.2. Finally, symmetry considerations allow the removal of some terms from each integral. To illustrate the procedure is presented in detail for the lateral hull quadratic lift and drag. Explicit expressions control foil and root lift and drag, and for longitudinal body crossflow drag are not given in the interest of space. 


\section{Example: Lateral Hull Lift and Drag}

The geometry relating hull sections in the $x y$-plane to the body-fixed coordinate frame is:

$$
\boldsymbol{R}_{b x y}=\left[\begin{array}{ccc}
0 & 1 & 0 \\
0 & 0 & 1 \\
1 & 0 & 0
\end{array}\right] \quad{ }^{b} r_{b x y}(z)=\left[\begin{array}{l}
0 \\
0 \\
z
\end{array}\right] .
$$

The differential quadratic lift and drag forces created by the hull in the $x y$-plane are then

$$
\begin{aligned}
d \boldsymbol{L}_{b x y} & =-\frac{1}{2} \rho K_{L_{b x y}} b_{b x y}\left[\begin{array}{c}
-(z p-v)^{2} \\
(z p-v)(z q+u) \\
0
\end{array}\right] d z \\
d \boldsymbol{D}_{b x y} & =-\frac{1}{2} \rho K_{D 0_{b x y}} b_{b x y} \sqrt{(u+z q)^{2}+(v-z p)^{2}}\left[\begin{array}{c}
u+z q \\
v-z p \\
0
\end{array}\right] d z
\end{aligned}
$$

The differential moments generated by these forces are related to the body-fixed coordinate frame by

$$
r_{C P_{b x y}}=\left[\begin{array}{lll}
y_{C P_{b x y}} & 0 & z
\end{array}\right]^{T},
$$

and are given by

$$
\begin{aligned}
d \boldsymbol{M}_{\boldsymbol{L}_{b x y}} & =-\frac{1}{2} \rho K_{L_{b x y}} b_{b x y}\left[\begin{array}{c}
z(-v+z p)(z q+u) \\
-z(-v+z p)^{2} \\
-y_{C P_{b x y}}(-v+z p)(z q+u)
\end{array}\right] d z \\
d \boldsymbol{M}_{\boldsymbol{D}_{b x y}} & =-\frac{1}{2} \rho K_{D o_{b x y}} b_{b x y}\left[\begin{array}{c}
-(v-z p) \\
z(z q+u) \\
y_{C P_{b x y}}(v-z p)
\end{array}\right] d z
\end{aligned}
$$


Noting that any integrand of odd order in $z$ is zero by the top/bottom symmetry of the body, the drag matrix associated with the $x y$-plane of the body is:

$$
\begin{aligned}
& \boldsymbol{D}_{\boldsymbol{b x \boldsymbol { y }} \boldsymbol{y}}=\frac{1}{2} \rho \int_{z} K_{L_{b x y}} b_{b x y}\left[\begin{array}{cccccc}
0 & -v & 0 & -z^{2} p & 0 & 0 \\
v & 0 & 0 & -z^{2} q & 0 & 0 \\
0 & 0 & 0 & 0 & 0 & 0 \\
z^{2} p & -z^{2} q & 0 & 0 & 0 & 0 \\
0 & 2 z^{2} p & 0 & 0 & 0 & 0 \\
y_{C P_{b x y}} v & 0 & 0 & -y_{C P_{b x y}} z^{2} q & 0 & 0
\end{array}\right] d z \\
&+\frac{1}{2} \rho \int_{z} K_{D_{b x y}} b_{b x y} \sqrt{(u+z q)^{2}+(v-z p)^{2}}\left[\begin{array}{cccccc}
1 & 0 & 0 & 0 & 0 & 0 \\
0 & 1 & 0 & 0 & 0 & 0 \\
0 & 0 & 0 & 0 & 0 & 0 \\
0 & 0 & 0 & z^{2} & 0 & 0 \\
0 & 0 & 0 & 0 & z^{2} & 0 \\
0 & y_{C P_{b x y}} & 0 & 0 & 0 & 0
\end{array}\right] d z
\end{aligned}
$$

The expressions for the $\boldsymbol{d}_{i}$ in equation (4.56) are simply the rows of the matrices above.

\subsection{Longitudinal Hull Lift Coefficients}

Five terms associated with longitudinal hull lift cannot be approximated using the strip-theory based approach of the previous section. Of these, four are potentially significant.

- Significant (associated with large horizontal velocity)

1. $Z_{u w_{b}}$ : longitudinal hull lift

2. $M_{u w_{b}}$ : longitudinal hull lift moment (portion not due to Munk moment)

3. $N_{u r_{b}}$ : yaw damping due to forward velocity

- Significant (associated with large vertical velocity)

1. $K_{w p_{b}}$ : roll damping due to vertical velocity

- Insignificant (associated with large vertical velocity)

1. $X_{u w_{b}}$ : forward hull lift

2. $M_{u w_{b}}$ : longitudinal hull lift moment (due to vertical velocity)

The latter two terms are small because the body geometry limits lift in the forward direction generated from non-zero pitch when moving primarily vertically. The significant terms above have been grouped according to the associated primary velocity. 
The first three are associated with primarily horizontal velocity, and as such cannot be included in a unified model where vertical velocity may dominate horizontal velocity. The opposite is true for $K_{w p_{b}}$.

We will limit our attention to the first three terms. Although Sentry will be capable of purely vertical translation, it will be at a significantly lower speed than its maximum forward speed. Thus, quadratic terms like those above will be small except when moving horizontally at speed.

The very small width to height ratio of the vehicle's frontal profile would invalidate any results derived from integration of the $2 \mathrm{D}$ hydrodynamic properties of longitudinal sections cut parallel to the $x z$-plane. For this reason, we turn to empirical results to estimate these coefficients. Note that the first two coefficients above have corresponding terms due to foil lift which have been modeled using the strip theory approach of the previous section; we seek only the contribution from the vehicle hull here.

Hoerner [9] provides empirical data for the lift and moment generated about a series of rotationally symmetric body streamlined body shapes. Although the body of our vehicle is not rotationally symmetric, we will use these coefficients to estimate the longitudinal body lift force and moment generated at small angles of attack (i.e. $u \approx$ $U_{o} ; w$ small). Hoerner provides the following coefficients for a rotationally symmetric body of similar cross-section.

$$
\begin{aligned}
& C_{Y_{\beta}}=1.2 \mathrm{rad}^{-1} \quad \text { where } \quad C_{Y_{\beta}}=\frac{d C_{Y}}{d \beta} \\
& C_{N_{\beta}}=-0.71 \mathrm{rad}^{-1} \quad \text { where } \quad C_{N_{\beta}}=\frac{d C_{N}}{d \beta} .
\end{aligned}
$$

Note that Hoerner gives his coefficients in ${ }^{\circ-1}$; the above values have been converted. In Hoerner's notation, these coefficients give the slope of the lateral force $Y$ and yaw moment $N$ versus side-slip angle $\beta$. We will employ them to estimate the longitudinal force $Z$ and pitching moment $M$ as follows:

$$
C_{Y}=\frac{-Z}{\frac{1}{2} \rho u^{2} d^{2}} \quad C_{N}=\frac{-M_{\text {midpt }}}{\frac{1}{2} \rho u^{2} L_{b} W_{b}^{2}} .
$$

$M_{\text {midpit }}$ represents the body lift moment measured at the lengthwise midpoint of the body. The preceding negative signs in both expressions above result from the fact that $Z_{w_{b}}$ is stabilizing (positive in Hoerner's notation, negative in ours) and $M_{\text {midpt }}$ is destabilizing (negative in Hoerner's notation, positive in ours because the z-axis 
points towards the sea-floor).

The side-slip angle $\beta$ becomes in our case, the angle of attack $\alpha$. For small angles of attack, $\alpha$ can be approximated as

$$
\alpha=\tan \left(\frac{w}{u}\right) \approx \frac{w}{u} .
$$

Thus, from 4.67,

$$
\begin{aligned}
Z_{w_{b}} & \approx-\frac{d}{d w}\left(\frac{1}{2} \rho u^{2} W_{b}^{2} C_{Y_{\beta}} \alpha\right)=-\frac{1}{2} \rho u d^{2} C_{Y_{\beta}} \\
Z_{u w_{b}} & =\frac{Z_{w_{b}}}{u}
\end{aligned}
$$

and

$$
\begin{aligned}
M_{\text {midpt }} & \approx-\frac{d}{d w}\left(\frac{1}{2} \rho u^{2} L_{b} W_{b}^{2} C_{N_{\beta}} \alpha\right) \\
& =-\frac{1}{2} \rho u L_{b} W_{b}^{2} C_{N_{\beta}} .
\end{aligned}
$$

The subscript $b$ is meant to identify these quantities as due to the hull of the vehicle alone. The body lift moment $M_{\text {midpt }}$ includes the contribution of the Munk moment given by

$$
M_{M u n k}=-\left(Z_{\dot{w}}-X_{\dot{u}}\right) u .
$$

The longitudinal Munk moment is a pure moment dependent only upon the added mass terms of the body. A numerical value was computed using strip theory in Section 4.1, thus we can compute the point at which the body lift force $Z_{w_{b}}$ acts on the body:

$$
x_{\text {midpt }}=\frac{M_{\text {Munk }}-M_{\text {midpt }}}{Z_{w_{b}}}
$$

where $x_{\text {midpt }}$ denotes the $x$-vector from the vehicle body midpoint to the point at which the lift force acts. For the Sentry vehicle it is negative indicating the body lift, due vortex shedding [9, 21], acts primarily along the rear half of the vehicle and is thus stabilizing. The distance from the body-fixed reference frame to the body midpoint is known, thus we can compute the component of body lift moment not due to the Munk moment:

$$
M_{u w_{b}}=\frac{-Z_{w_{b}}\left(x_{c . l .}\right)}{u}
$$

where $x_{c . l .}$. denotes the $\mathrm{x}$-vector to the longitudinal center of lift from the origin of the body-fixed reference frame. Because $x_{c . l}<0$ for the Sentry vehicle, the body lift 
Table 4.7: Longitudinal Hull Lift Coefficients

\begin{tabular}{crlll}
\hline \hline Parameter & Value & Units & Description \\
\hline$Z_{u w_{b}}$ & -391 & $\mathrm{~kg} / \mathrm{m}$ & longitudinal body lift force \\
$M_{u w_{b}}$ & -208 & $\mathrm{~kg}$ & longitudinal body lift moment \\
$N_{u r_{b}}$ & $?$ & $\mathrm{~kg}$ & lateral rotational drag at speed \\
$K_{w p_{b}}$ & $?$ & $\mathrm{~kg}$ & roll drag for high vertical velocity \\
\hline \hline
\end{tabular}

moment $M_{u w_{b}}$ is stabilizing.

Note that the net effect, including contributions from the body lift moment, Munk moment, and foil lift moments is slightly stabilizing for the Sentry vehicle. Thus we would expect the vehicle to become more damped in pitch as vehicle speed increases (see Chapter 5 for a complete discussion).

No reliable means was found to estimate $N_{u r_{b}}$, the increased damping in yaw expected at significant forward speed.

Values for these coefficients are given in Table 4.7.

\subsubsection{Linear Damping}

A second order Taylor expansion of hydrodynamic drag forces about zero velocity results in linear terms and quadratic terms. At velocities sufficiently far from zero, drag (and lift) effects are well modeled by the quadratic terms discussed in detail in the preceding sections. For a vehicle whose nominal operating condition includes significant forward speed, these quadratic terms may be sufficient; however, since we are potentially interested in the hover and low speed behavior of the Sentry AUV, we must consider the linear terms as well. Indeed, if the drag forces were only quadratic in nature, damping at low speeds would be insignificant and experience clearly demonstrates the contrary.

It is tempting to regard the linear term of the Taylor series as a direct consequence of purely viscous drag which does indeed have a linear characteristic at very low Reynolds Numbers, $R n<1$. This linearity can be verified directly by dimensional analysis under the assumption that at very low $R n$, the inertia of the fluid, and thus its density $\rho$, has no bearing on the forces within that fluid. In practice, the linear terms of the Taylor Series instead represent a crude approximation to the $R n$-dependence of the fluid drag forces. As such, these terms must be determined experimentally.

Once estimates for these terms become available, they can be conveniently expressed as linear stability derivatives (e.g. $X_{u}$ ) and inserted into a constant matrix 
of linear damping terms $\boldsymbol{D}_{\text {lin }}$.

\subsection{Hydrostatic Forces}

For a properly trimmed, neutrally buoyant vehicle of wet weight $W$, the hydrostatic forces exert only pitch and roll righting moments. For the Sentry vehicle, the vector of gravitational restoring forces $\boldsymbol{g}(\boldsymbol{\eta})$ is given by

$$
\boldsymbol{g}(\boldsymbol{\eta})=\left[\begin{array}{c}
0 \\
0 \\
0 \\
z_{B G} \cos \theta \sin \phi W \\
z_{B G} \sin \theta W \\
0
\end{array}\right]
$$

where $z_{B G}$ is defined in body-fixed coordinates as the vertical distance between the center of buoyancy and the center of mass.

Note that the validity of this expression is not compromised by the additional mass of entrained water typical of flooded AUV hulls. For symmetric AUV hulls, it is typically trivial to locate the center of buoyancy by symmetry considerations alone, although such a procedure is predicated on the assumption that entrained water acts as though it were rigidly attached to the hull. In contrast, the center of mass of a flooded vehicle can be difficult to determine directly because of the complexity of the flooded space. For a neutrally buoyant and properly trimmed vehicle it is enough to determine the CB/CG separation of the dry vehicle, where the CB is calculated as if all components were composed purely of water, as this quantity will not change upon immersion. Specifically,

$$
\boldsymbol{r}_{\boldsymbol{G}_{\text {wet }}}=\boldsymbol{r}_{\boldsymbol{B}_{\boldsymbol{w e t}}}+z_{B G_{d r y}} \hat{k}
$$

which implies

$$
z_{B G_{w e t}}=z_{B G_{d r y}}
$$

The potentially significant mass of the control foils raises the possibility that gravitational restoring forces, and indeed the location of the center of mass of the complete vehicle will change with changing foil position. Although this effect cannot be accounted for dynamically in the 6 degree-of-freedom model we are pursuing here, the equations necessary to derive the gravitational force vector for arbitrary static foil position are given in Appendix A. Fortunately, this effect is likely to be minimal 
or nonexistent since to eliminate static torque requirements, the center of mass of the foils should be coincident with the foil axis.

\subsection{Thruster and Control Foil Interaction}

Throughout the preceding development, we have implicitly assumed that the thrusters do not interact with the foils to alter the lift and drag forces that would be produced in free stream flow. In fact, the both the lift and drag of the foils is influenced by the additional velocity imparted to the fluid flowing over the foils by the thrusters.

The steady-state increased flow velocity parallel to the foil chord (perpendicular to the surface described by the rotating props), produces two conflicting effects in terms of the lift produced by the foils. The increased fluid velocity acts to increase foil lift, but concurrently reduces the effective angle of attack of the foils, thus acting to reducing lift. The parasitic drag of the foils is increased slightly because of the increase in flow velocity, but this effect is minor.

We show that based on a steady state actuator disk analysis, these effects approximately cancel out and can be safely ignored. No attempt has been made to consider the rotational inertia imparted to the fluid by the rotating propellers, nor have we considered dynamic effects.

We begin by briefly summarizing the derivation and results obtained by approximating a propeller in free flow as an actuator disk (e.g. [3], pg. 200-203). The goal of our analysis is to arrive at an expression for the approximate velocity of the fluid across the propeller as a function of applied thrust and advance velocity.

By repeated application of mass conservation, conservation of linear momentum, and the Bernoulli equation for incompressible flow it can be shown that

$$
\begin{aligned}
& \frac{\rho}{2}\left(V_{w}^{2}-V_{a}^{2}\right)=\frac{T}{A} \\
& V_{p}=\frac{1}{2}\left(V_{w}+V_{a}\right)
\end{aligned}
$$

where $T$ denotes the propeller thrust, $A_{p}$ denotes the area described by the rotating propeller (the actuator disk), and the fluid velocities are as labeled in Figure 4-2.

Solving (4.80) for $V_{w}$ and substituting the result into (4.79) gives:

$$
\left(4 V_{p}^{2}-4 V_{p} V_{a}+V_{a}^{2}-V_{a}^{2}\right)=\frac{2 T}{\rho A}
$$




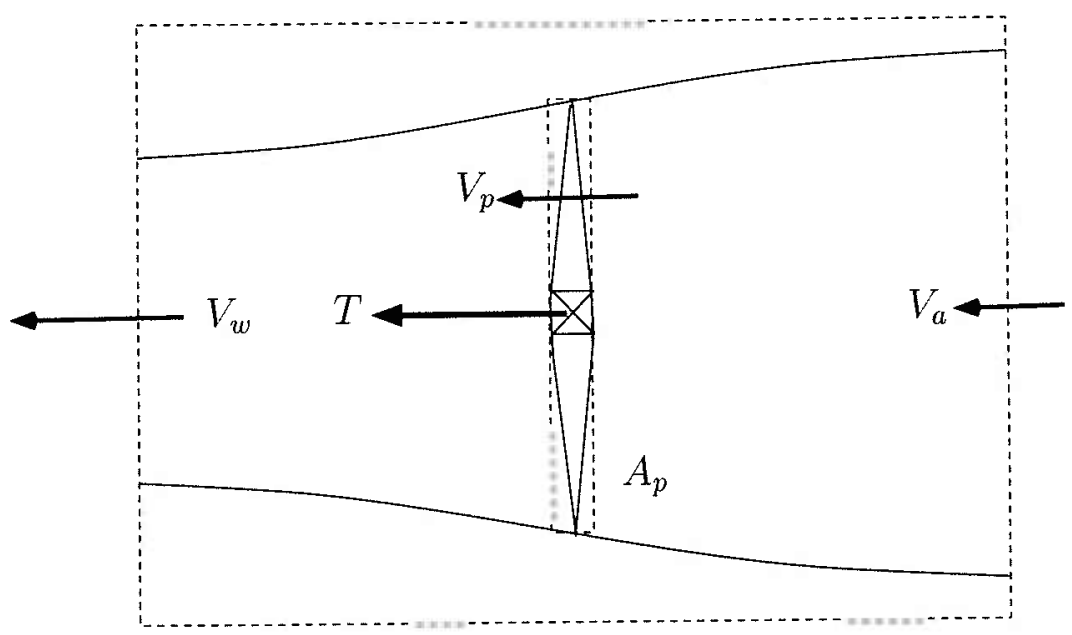

Figure 4-2: Classical Actuator Disk Analysis, figure adapted from Fay [3].

Solving for $V_{p}$ under the physical condition that $V_{p}>0$ gives the desired relation:

$$
V_{p}=\frac{V_{a}+\sqrt{V_{a}^{2}+\frac{2 T}{\rho A}}}{2} .
$$

To extend these results to the combined foil/thruster actuators on the Sentry vehicle, we make the following assumptions:

1. The flow over the foils is two-dimensional.

2. The flow over the foils is uniform in velocity

3. The velocity of the fluid over the foils is equal to the steady-state fluid velocity at the propeller $V_{p}$.

4. The foils will operate at low angle of attack.

5. The actuator disk analysis above applies in a fluid velocity vector addition sense.

The first assumption is consistent with our treatment of the foils as two-dimensional in the preceding sections. The second and third represent the worst case, in that the lower fluid velocity ahead of the props should have a decreased effect on the lift of the foils. The forth and fifth effectively assume that the actuator disk analysis applies, even in the presence of small cross-flow velocities. That is, we assume (4.82) applies to the fluid velocity vector components perpendicular to the plane of the propeller. We apply this analysis only to small angles of attack as it is well documented (e.g. 


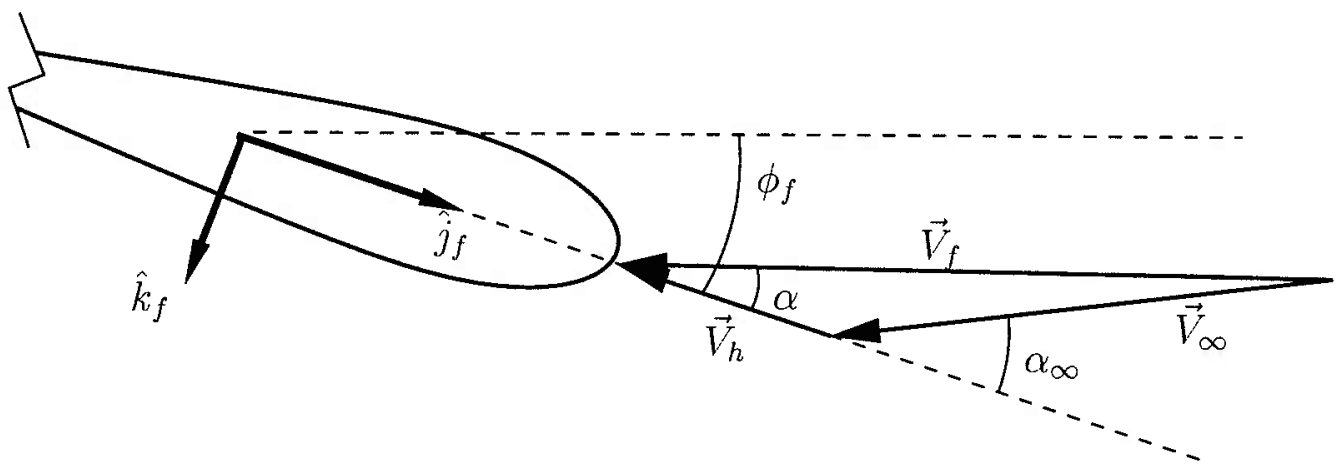

Figure 4-3: Thruster and Control Foil Interaction, fluid velocity vector definitions

[22]) that thruster performance is severely degraded in significant cross-flows. This latter fact does not compromise the utility of our analysis since the Sentry vehicle was specifically designed to avoid operating in configurations and speeds where the thrusters would experience significant cross-flow.

Under these assumptions, we can view the effect of the thrusters on foil lift and drag in terms of the vector addition of the free stream velocity $\vec{V}_{\infty}$ and the thrusterinduced fluid velocity $\vec{V}_{i}$, where

$$
\vec{V}_{i}=-\left(V_{p}-v_{a}\right) \dot{j} j_{f}
$$

The resultant effective fluid velocity vector $V_{f}$ determines both the resulting angle of attack and fluid velocity across the foil (Figure 4-3.

Since ultimately we are concerned with the validity of our model from a control design perspective, it makes sense to consider the effect of propeller/foil interaction in terms of its effect on physical "lift gain." That is, we consider it in terms its effect on the increase in lift incurred by a unit change of foil angle:

$$
\text { "lift gain" }=\frac{\delta L}{\delta \phi_{f}} .
$$

For small angles of attack, the dependence of the lift generated by a foil on angle of attack $\alpha$ is well approximated by the linear relationship

$$
L=\frac{1}{2} \rho\left|\vec{V}_{f}\right|^{2} A_{f} K_{L} \alpha
$$

where $A_{f}$ is the planform area of the foil, and $K_{L}$ the slope of the lift coefficient vs. $\alpha$ curve at $\alpha=0$. Based on Figure 4-3, both $\vec{V}_{f}$ and $\alpha$ are affected by the foil angle 
$\phi_{f}$.

Taking the derivative of (4.85) with respect to foil angle yields the lift gain:

$$
\frac{\delta L}{\delta \phi_{f}}=\frac{1}{2} \rho A_{f} K_{L}\left(2 V_{f} \frac{\delta V_{f}}{\delta \phi_{f}} \alpha+V_{f}^{2} \frac{\delta \alpha}{\delta \phi_{f}}\right) .
$$

Expressions for $V_{f}$ and $\alpha$ are available from the geometry in Figure 4-3. For simplicity, but without loss of generality, we consider the case $V_{\infty}=U_{o} \hat{i}$. It follows then that

$$
V_{a}=-V_{\infty} \cdot \hat{j}_{f}=U_{o} \cos \phi_{f}
$$

By definition, $\vec{V}_{i}=-\left(V_{p}-V_{a}\right) \cdot \hat{j}_{f}$. Substituting in the result for $V_{p}$ from 4.82 yields

$$
\vec{V}_{i}=\frac{1}{2}\left(V_{a}-\sqrt{V_{a}^{2}+\frac{2 T}{\rho A_{p}}}\right) \hat{j}_{f}
$$

which upon substitution for $V_{a}$ and transformation into body frame coordinates yields:

$$
\vec{V}_{i}=\frac{1}{2}\left(U_{o} \cos \phi_{f}-\sqrt{U_{o}^{2} \cos ^{2} \phi_{f}+\frac{2 T}{\rho A_{p}}}\right)\left(\cos \phi_{f} \hat{i}+\sin \phi_{f} \hat{k}\right)
$$

Finally,

$$
\begin{aligned}
\vec{V}_{f}= & \vec{V}_{\infty}+\vec{V}_{i} \\
= & \left\{-U_{o}+\frac{1}{2}\left(U_{o} \cos \phi_{f}-\sqrt{U_{o}^{2} \cos ^{2} \phi_{f}+\frac{2 T}{\rho A_{p}}}\right) \cos \phi_{f}\right\} \hat{i} \\
& +\frac{1}{2}\left\{U_{o} \cos \phi_{f}-\sqrt{U_{o}^{2} \cos ^{2} \phi_{f}+\frac{2 T}{\rho A_{p}}}\right\} \sin \phi_{f} \hat{k} .
\end{aligned}
$$

After simplification, the magnitude squared is

$$
\left|\vec{V}_{f}\right|^{2}=\frac{1}{2}\left(4 U_{o}^{2}-2 U_{o}^{2} \cos ^{2} \phi_{f}+2 U_{o} \cos \phi_{f} \sqrt{U_{o}^{2} \cos ^{2} \phi_{f}+\frac{2 T}{\rho A_{p}}}+\frac{2 T}{\rho A_{p}}\right)
$$

Referring back to Figure 4-3, the angle of attack $\alpha$ is

$$
\cos \alpha=\frac{-\vec{V}_{f} \cdot \hat{j}_{f}}{\left|\vec{V}_{f}\right|}=\frac{U_{o} \cos \phi_{f}+\sqrt{U_{o}^{2} \cos ^{2} \phi_{f}+\frac{2 T}{\rho A_{p}}}}{2\left|\vec{V}_{f}\right|}
$$

From this point forward it is straightforward to compute the components of 4.85 . No 
special insight is offered by these algebraically complex expressions and we omit them here. Instead the results of this analysis are shown graphically in Figure 4-4.

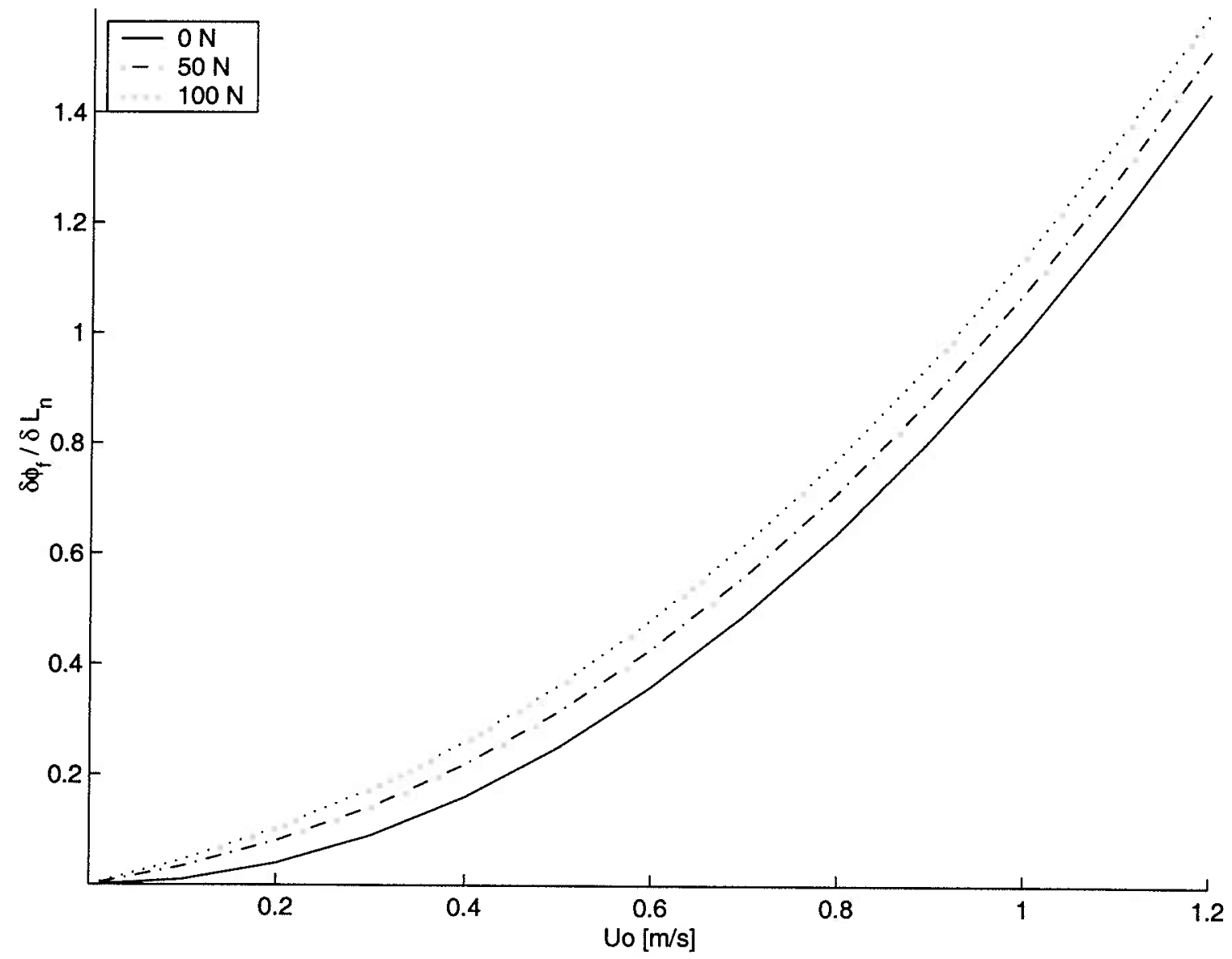

Figure 4-4: Normalized "lift gain" $\frac{\delta L}{\delta \phi_{f}}$ as a function of nominal forward speed $U_{o}$ for representative thrust levels. To generate the plot, the lift $L$ was normalized such that $L=\left(\frac{1}{2} \rho A_{f} K_{L}\right) L_{n}$. The propeller area used was $A_{p}=0.3 \mathrm{~m}^{2}$.

The solid curve in Figure 4-4 represents the lift gain at zero thrust as a function of forward speed, and shows the behavior of the model as derived without consideration of propeller/foil interaction. It is evident from the small deviations of the other curves relative to the blue curve that the interaction between the foils and thrusters can be ignored. 


\section{Chapter 5}

\section{Simplified Models}

In this chapter we consider the six degree-of-freedom linearized dynamics of the Sentry AUV. The general linearization procedure is presented in the following section. The explicit results for nominally horizontal flight are given to facilitate linear controller design and for use as a simplified vehicle dynamics model for pre-mission simulations.

We also consider some of the complications arising from the non-linear mapping of the inputs to the vehicle axes in the context of near vertical flight. Because the thrusters are mounted on the vehicle's rotating control foils, foil lift and propeller thrust map to the vehicle axes in a manner that is dependent upon foil angle.

\subsection{General Linearization Procedure}

The linearization procedure is straightforward and is outlined in the following section; however as is shown, the non-linear mapping of the thruster and foil inputs complicates the procedure for arbitrary nominal velocity. We consider only translational equilibrium velocities in the vertical plane and nonzero equilibrium pitch, as all other potential equilibrium conditions are irrelevant to the vehicle's mission profile (e.g. non-zero roll), or do not affect the vehicle's dynamics (e.g. yaw angle).

The non-linear six-degree of freedom equations of motion expressed in the bodyfixed frame were derived in the preceding sections and can be written

$$
\begin{gathered}
M \dot{\nu}+C(\nu) \nu+D\left(\nu, \phi_{f}\right) \nu+g(\eta)=b\left(\phi_{f}, h\right) \\
\dot{\eta}=J \nu .
\end{gathered}
$$

Defining $f_{C}=C \boldsymbol{\nu}$ and $f_{D}=D \nu$, the Taylor expansion of (5.1) to first order is 
then:

$$
\begin{aligned}
\boldsymbol{M} \Delta \boldsymbol{\nu}+\left.\frac{\delta \boldsymbol{f}_{\boldsymbol{C}}}{\delta \boldsymbol{\nu}}\right|_{\boldsymbol{\nu}_{o}} \Delta \boldsymbol{\nu}+\left.\frac{\delta \boldsymbol{f}_{\boldsymbol{D}}}{\delta \boldsymbol{\nu}}\right|_{\boldsymbol{\nu}_{o}, \boldsymbol{\phi}_{f_{o}}} \Delta \boldsymbol{\nu}+\left.\frac{\delta \boldsymbol{g}}{\delta \boldsymbol{\eta}}\right|_{\boldsymbol{\eta}_{o}} \Delta \boldsymbol{\eta}= \\
\quad\left(\left.\frac{\delta \boldsymbol{f}_{\boldsymbol{D}}}{\delta \boldsymbol{\phi}_{f}}\right|_{\boldsymbol{\nu}_{o}, \boldsymbol{\phi}_{f_{o}}}+\left.\frac{\delta \boldsymbol{b}}{\delta \phi_{f}}\right|_{\phi_{f_{o}}, \boldsymbol{h}_{o}}\right) \Delta \boldsymbol{\phi}_{f}+\left.\frac{\delta \boldsymbol{b}}{\delta \boldsymbol{h}}\right|_{\phi_{f_{o}}, \boldsymbol{h}_{o}} \Delta \boldsymbol{h}
\end{aligned}
$$

where $\Delta c=c-c_{o}$ for generic vector $c$, and where we have moved linear terms associated with foil angles to the right side as inputs.

The linearized kinematics of the vehicle are similarly derived from their nonlinear counterpart:

$$
\dot{\eta}=\boldsymbol{J}(\boldsymbol{\eta}) \boldsymbol{\nu}
$$

Linearizing as before,

$$
\dot{\boldsymbol{\eta}}_{o}+\Delta \dot{\boldsymbol{\eta}} \approx \boldsymbol{J}\left(\boldsymbol{\eta}_{o}\right) \boldsymbol{\nu}_{o}+\left.\frac{\delta(\boldsymbol{J}(\boldsymbol{\eta}) \boldsymbol{\nu})}{\delta \boldsymbol{\nu}}\right|_{\boldsymbol{\nu}_{o}, \boldsymbol{\eta}_{o}} \Delta \boldsymbol{\nu}+\left.\frac{\delta(\boldsymbol{J}(\boldsymbol{\eta}) \boldsymbol{\nu})}{\delta \boldsymbol{\eta}}\right|_{\boldsymbol{\nu}_{o}, \boldsymbol{\eta}_{o}} \Delta \boldsymbol{\eta}
$$

From (5.3), we see that the constant terms on either side cancel, and we are left with

$$
\Delta \dot{\eta} \approx J\left(\boldsymbol{\eta}_{o}\right) \Delta \nu+\left.\frac{\delta \boldsymbol{J}(\boldsymbol{\eta})}{\delta \boldsymbol{\eta}}\right|_{\eta_{o}} \boldsymbol{\nu}_{o} \Delta \boldsymbol{\eta}
$$

By defining a new 12-element state vector $\boldsymbol{x}=(\Delta \boldsymbol{\nu}, \Delta \boldsymbol{\eta})^{T}$ and 6-element input vector $\boldsymbol{u}=\left(\Delta \boldsymbol{\phi}_{\boldsymbol{f}}, \Delta \boldsymbol{h}\right)^{T}$, the linearized equations of motion can be written in the familiar form $\dot{\boldsymbol{x}}=\boldsymbol{A} \boldsymbol{x}+\boldsymbol{B} \boldsymbol{u}$ as follows:

$$
\dot{\boldsymbol{x}}=\left[\begin{array}{cc}
-M^{-1}(C+D) & -M^{-1} G \\
J & \left.\frac{\delta J(\eta)}{\delta \eta}\right|_{\eta_{o}}
\end{array}\right] x+\left[\begin{array}{c}
\boldsymbol{\nu}_{o}^{-1} B \\
0
\end{array}\right] u .
$$

Note that in (5.1) we have assumed implicitly that the zero-order terms cancel:

$$
C\left(\nu_{o}\right) \nu_{o}+D\left(\nu_{o}, \phi_{f_{o}}\right)+g\left(\eta_{o}\right)=b\left(\phi_{f_{o}}, h_{o}\right)
$$

The solution to (5.7) for general $\boldsymbol{\nu}_{\boldsymbol{o}}, \boldsymbol{\eta}_{\boldsymbol{o}}$ is algebraically complex. Limiting $\boldsymbol{\nu}_{\boldsymbol{o}}, \boldsymbol{\eta}_{o}$ to velocities and orientations of interest, specifically,

$$
\boldsymbol{\nu}_{o}=\left[\begin{array}{llllll}
U_{o} & W_{o} & 0 & 0 & 0 & 0
\end{array}\right]^{T} \quad \boldsymbol{\eta}_{o}=\left[\begin{array}{llllll}
0 & 0 & 0 & 0 & \Theta_{o} & 0
\end{array}\right]^{T}
$$


reduces the complexity of (5.7) considerably, but we are still left with a system of nonlinear equations for the equilibrium inputs, $\phi_{f_{o}}$ and $\boldsymbol{h}_{o}$. This complexity is a result of the non-linear structure of the inputs (see 3.15). A solution to these nominal inputs is necessary to verify the existence of an equilibrium point at the given nominal velocities, without which a linearized analysis makes little sense. The foil angles and thrusts must be chosen such that the resultant lift, drag and thrust vectors from the foils exactly balance the hydrodynamic force and moment on the rest of the vehicle.

An explicit system of equations for $\phi_{f_{o}}$ and $h_{o}$ result from evaluating the nonlinear equations of motion (5.1) at $\boldsymbol{\nu}=\boldsymbol{\nu}_{o}$ and $\boldsymbol{\eta}=\boldsymbol{\eta}_{o}$. No general solution to this algebraically complex system of equations is attempted here. However, the solution for near-horizontal flight $\left(u \approx U_{o}, w \approx 0\right)$ is intuitive and algebraically trivial. The derivation of the linearized equations of motion for this operating condition is the subject of the next section.

\subsection{Near Horizontal Flight}

For near horizontal flight $u \approx U o$ and all other velocities are considered to be small. For efficient flight, the foils should be nominally flat, i.e. $\phi_{f} \approx 0$. Thus, $(5.7)$ reduces to:

$$
\left[\begin{array}{c}
h_{f p_{o}}+h_{f s_{o}}+h_{a p_{o}}+h_{a s_{o}} \\
0 \\
0 \\
0 \\
0 \\
b_{t}\left(h_{f p_{o}}-h_{f s_{o}}+h_{a p_{o}}-h_{a s_{o}}\right)
\end{array}\right]=\left[\begin{array}{c}
X_{o} \\
0 \\
0 \\
0 \\
0 \\
0
\end{array}\right]
$$

where $X_{o}$ represents the nominal vehicle drag at $u \approx U o$.

The last row of (5.9) requires $h_{f p_{o}}+h_{a p_{o}}=h_{f s_{o}}+h_{a s_{o}}$. To prevent roll excitation upon deviation from $\phi_{f}=0$ we set the nominal thrusts of each thruster-pair equal:

$$
\begin{aligned}
& h_{f p_{o}}=h_{f s_{o}} \\
& h_{a p_{o}}=h_{a s_{o}} .
\end{aligned}
$$

Under these two conditions, we still have the freedom to set the forward thruster-pair to aft thruster-pair nominal thrust ratio such that the sum equals $X_{o}$. We define:

$$
\beta_{h}=\frac{h_{f p_{o}}+h_{f s_{o}}}{h_{a p_{o}}+h_{a s_{o}}}
$$


which yields,

$$
\begin{gathered}
h_{f}=\frac{\beta_{h} X_{o}}{1+\beta_{h}}=2 h_{f p_{o}}=2 h_{f s_{o}} \\
h_{a}=\frac{X_{o}}{1+\beta_{h}}=2 h_{a p_{o}}=2 h_{a s_{o}} .
\end{gathered}
$$

As will be shown, $\beta_{h}$ affects control over vehicle pitch; moreso for large $X_{o}$.

Evaluating the terms of (5.1) and (5.4) under these conditions gives the following expressions for the components of the linearized equations of motion about nominally horizontal flight.

$$
\begin{aligned}
& \boldsymbol{M}_{\boldsymbol{R} \boldsymbol{B}}=\left[\begin{array}{cccccc}
m & 0 & 0 & 0 & m z_{G} & 0 \\
0 & m & 0 & -m z_{G} & 0 & 0 \\
0 & 0 & m & 0 & 0 & 0 \\
0 & -m z_{G} & 0 & I_{x x} & 0 & -I_{x z} \\
m z_{G} & 0 & 0 & 0 & I_{y y} & 0 \\
0 & 0 & 0 & -I_{x z} & 0 & I_{z z}
\end{array}\right] \quad \boldsymbol{C}_{\boldsymbol{R} B}=\left[\begin{array}{cccccc}
0 & 0 & 0 & 0 & 0 & 0 \\
0 & 0 & 0 & 0 & 0 & 0 \\
0 & 0 & 0 & 0 & -m U_{o} \\
0 & 0 & 0 & 0 & 0 \\
0 & 0 & 0 & 0 & 0 & 0 \\
0 & 0 & 0 & 0 & 0 & 0 \\
0 & 0 & 0 & 0 \\
z_{G} U_{o} \\
0
\end{array}\right] \\
& \boldsymbol{M}_{\boldsymbol{A}}=-\left[\begin{array}{cccccc}
X_{\dot{u}} & 0 & 0 & 0 & 0 & 0 \\
0 & Y_{\dot{v}} & 0 & 0 & 0 & N_{\dot{v}} \\
0 & 0 & Z_{\dot{w}} & 0 & M_{\dot{w}} & 0 \\
0 & 0 & 0 & K_{\dot{p}} & 0 & 0 \\
0 & 0 & M_{\dot{w}} & 0 & M_{\dot{q}} & 0 \\
0 & N_{\dot{v}} & 0 & 0 & 0 & N_{\dot{r}}
\end{array}\right] \quad \boldsymbol{C}_{\boldsymbol{A}}=\left[\begin{array}{cccccc}
0 & 0 & 0 & 0 & 0 & 0 \\
0 & 0 & 0 & 0 & 0 & -X_{\dot{u}} U_{o} \\
0 & 0 & 0 & 0 & X_{\dot{u}} U_{o} & 0 \\
0 & 0 & 0 & 0 & 0 \\
0 & 0 & \left(Z_{\dot{w}}-X_{\dot{u}}\right) U_{o} & 0 & M_{\dot{w}} U_{o} & 0 \\
0 & 0 & 0 & 0 & -X_{\dot{u}} U_{o}
\end{array}\right] \\
& \boldsymbol{D}=-\left[\begin{array}{cccccc}
X_{u} & 0 & 0 & 0 & 0 & 0 \\
0 & Y_{v} & 0 & 0 & 0 & 0 \\
0 & 0 & Z_{w} & 0 & Z_{q} & 0 \\
0 & 0 & 0 & K_{p} & 0 & 0 \\
0 & 0 & M_{w} & 0 & M_{q} & 0 \\
0 & N_{v} & 0 & 0 & 0 & N_{r}
\end{array}\right] \quad \boldsymbol{G}=\left[\begin{array}{cccccc}
0 & 0 & 0 & 0 & 0 & 0 \\
0 & 0 & 0 & 0 & 0 & 0 \\
0 & 0 & 0 & 0 \\
0 & 0 & 0 & 0 \\
0 & 0 & 0 & 0 & 0 \\
0 & 0 & 0 & 0 & 0 & 0 \\
0 & 0 & 0 & 0 \\
\left(z_{G}-z_{B}\right) W & 0 \\
0 & 0 & 0
\end{array}\right] \\
& \boldsymbol{B}_{\boldsymbol{h}}=\left[\begin{array}{cccc}
1 & 1 & 1 & 1 \\
0 & 0 & 0 & 0 \\
0 & 0 & 0 & 0 \\
0 & 0 & 0 & 0 \\
0 & 0 & 0 \\
b_{h} & -b_{h} & b_{h} & -b_{h}
\end{array}\right] \quad \boldsymbol{B}_{\boldsymbol{f}}=\left[\begin{array}{cc}
0 & 0 \\
0 & 0 \\
2 \frac{\beta_{h}}{1+\beta_{h}} X_{o}+Z_{\phi_{f f}} & 2 \frac{1}{1+\beta_{h}} X_{o}+Z_{\phi_{a f}} \\
-2 a_{f f} \frac{\beta_{h}}{1+\beta_{h}} X_{o}+M_{\phi_{f f}} & -2 a_{a f} \frac{1}{1+\beta_{h}} X_{o}+M_{\phi_{a f}} \\
0 & 0
\end{array}\right] \\
& \boldsymbol{J}\left(\boldsymbol{\eta}_{o}\right)=\left.I_{6 \times 6} \quad \frac{\delta \boldsymbol{J}(\boldsymbol{\eta})}{\delta \boldsymbol{\eta}}\right|_{\boldsymbol{\eta}_{o}} \boldsymbol{\nu}_{o}=\left[\begin{array}{cccc}
0_{3 \times 3} & 0 & 0 & 0 \\
& 0 & -U_{o} & 0 \\
0_{3 \times 3} & 0_{3 \times 3}
\end{array}\right]
\end{aligned}
$$


The hydrodynamic lift and drag derivatives above are:

$$
\begin{aligned}
& X_{o}=\frac{1}{2} \rho\left|U_{o}\right| U_{o} \int_{z} K_{D_{b x y}} b_{b x y} d z+\rho\left|U_{o}\right| U_{o} \int_{x_{f f, a f, f r, a r}} K_{D_{o s}} b_{s} d x_{s} \\
& X_{u}=X_{u_{l i n}}-\rho\left|U_{o}\right| \int_{z} K_{D_{b x y}} b_{b x y} d z-\rho\left|U_{o}\right| \int_{x_{f f, a f, f r, a r}} K_{D_{o s}} b_{s} d x_{s} \\
& Y_{v}=Y_{v_{l i n}}-\frac{1}{2} \rho U_{o} \int_{z} K_{L_{b x y}} b_{b x y} d z-\frac{1}{2} \rho\left|U_{o}\right| \int_{z} K_{D_{b x y}} b_{b x y} d z \\
& Z_{w}=Z_{w_{l i n}}+Z_{u w_{b}} U_{o}-\frac{1}{2} \rho\left|U_{o}\right| \int_{x_{f f, a f, f r}, a r}\left(K_{L_{s}}+K_{D_{o_{s}}}\right) b_{s} d x_{s} \\
& Z_{q}=\frac{1}{2} \rho \int_{x_{f f, f r}}\left(K_{L_{s}}+K_{D_{o_{s}}}\right) b_{s} a_{f f} d x_{s}+\frac{1}{2} \rho \int_{x_{a f, a r}}\left(K_{L_{s}}+K_{D_{o s}}\right) b_{s} a_{a f} d x_{s} \\
& Z_{\phi_{f f, a f}}=\frac{1}{2} \rho\left|U_{o}\right| U_{o} \int_{x_{f f, a f}} K_{L_{s}} b_{s} d x_{s} \\
& K_{p}=K_{p_{l i n}}-\frac{1}{2} \rho U_{o} \int_{z} K_{L_{b x y}} b_{b x y} z^{2} d z-\frac{1}{2} \rho\left|U_{o}\right| \int_{z} K_{D_{b x y}} b_{b x y} z^{2} d z \\
& -\frac{1}{2} \rho\left|U_{o}\right| \int_{x_{f f, a f, f r, a r}}\left(K_{L_{s}}+K_{D_{o_{s}}}\right) b_{s} x_{s}^{2} d x_{s} \\
& M_{q}=M_{q_{l i n}}-\rho\left|U_{o}\right| \int_{z} K_{D_{b x y}} b_{b x y} z^{2} d z \\
& -\frac{1}{2} \rho\left|U_{o}\right| \int_{x_{f f, f r}}\left(a_{f f}\left(a_{f f}+y_{C P_{s}}\right)\left(K_{L_{s}}+K_{D_{o_{s}}}\right)\right) b_{s} d x_{s} \\
& -\frac{1}{2} \rho\left|U_{o}\right| \int_{x_{a f, a r}}\left(a_{a f}\left(a_{a f}+y_{C P_{s}}\right)\left(K_{L_{s}}+K_{D_{o s}}\right)\right) b_{s} d x_{s} \\
& M_{w}=M_{u w_{b}} U_{o}+\frac{1}{2} \rho\left|U_{o}\right| \int_{x_{f f, f r}}\left(a_{f f}+y_{C P_{s}}\right)\left(K_{L_{s}}+K_{D_{o_{s}}}\right) b_{s} d x_{s} \\
& +\frac{1}{2} \rho\left|U_{o}\right| \int_{x_{a f, a r}}\left(a_{a f}+y_{C P_{s}}\right)\left(K_{L_{s}}+K_{D_{o s}}\right) b_{s} d x_{s} \\
& M_{\phi_{f f, a f}}=-\frac{1}{2} \rho\left|U_{o}\right| U_{o} \int_{x_{f f, a f}}\left(\left(a_{s}+y_{C P_{s}}\right) K_{L_{s}}+y_{C P_{s}} K_{D_{o s}}\right) b_{s} d x_{s} \\
& N_{r}=N_{r_{l i n}}+N_{u r_{b}} U_{o}-\rho\left|U_{o}\right| \int_{x_{f f, a f, f r, a r}} K_{D_{o s}} b_{s} x_{s}^{2} d x_{s} \\
& N_{v}=-\frac{1}{2} \rho U_{o} \int_{z}\left(K_{L_{b x y}}+K_{D_{b x y}}\right) b_{b x y} y_{C P_{b x y}} d z
\end{aligned}
$$

where the multiple integral subscripts indicate integration along the $x$-axes of all the indicated vehicle components.

Assuming $U_{o}>0$, ignoring linear drag terms significant only at low speed, and factoring out $U_{o}$ and $\beta_{h}$ from the expressions above where possible, we can make 
the static dependence of the linearized equations of motion on nominal speed and $\beta_{h}$ explicit. Substituting the terms of (5.13) into (5.6) yields the linearized equations of motion about nominally horizontal flight in standard form $\dot{\boldsymbol{x}}=\boldsymbol{A} \boldsymbol{x}+\boldsymbol{B} \boldsymbol{u}$ where

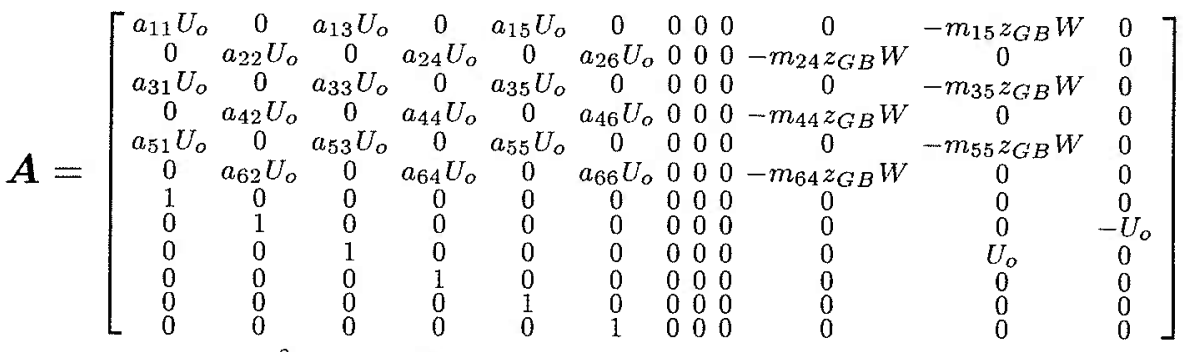

$$
\begin{aligned}
& \boldsymbol{B}=\left[\begin{array}{cccccc}
\frac{\beta_{h}}{1+\beta_{h}} h_{11}+U_{o}^{2} f_{11} & \frac{1}{1+\beta_{h}} h_{12}+U_{o}^{2} f_{12} & m_{11} & m_{11} & m_{11} & m_{11} \\
0 & 0 & m_{26} b_{t} & -m_{26} b_{t} & m_{26} b_{t} & -m_{26} b_{t} \\
\frac{\beta_{h}}{1+\beta_{h}} h_{31}+U_{o}^{2} f_{31} & \frac{1}{1+\beta_{h}} h_{32}+U_{o}^{2} f_{32} & m_{31} & m_{31} & m_{31} & m_{31} \\
0 & 0 & m_{46} b_{t} & -m_{46} b_{t} & m_{46} b_{t} & -m_{46} b_{t} \\
\frac{\beta_{h}}{1+\beta_{h}} h_{51}+U_{o}^{2} f_{51} & \frac{1}{1+\beta_{h}} h_{52}+U_{o}^{2} f_{52} & m_{51} & m_{51} & m_{51} & m_{51} \\
0 & 0 & m_{66} b_{t} & -m_{66} b_{t} & m_{66} b_{t} & -m_{66} b_{t} \\
0 & 0 & 0 & 0 & 0 & 0 \\
0 & 0 & 0 & 0 & 0 & 0 \\
0 & 0 & 0 & 0 & 0 & 0 \\
0 & 0 & 0 & 0 & 0 & 0 \\
0 & 0 & 0 & 0 & 0 & 0
\end{array}\right]
\end{aligned}
$$

In the matrices above, $m_{i j}$ represent the individual terms of the inverse matrix $M^{-1}$. The remaining $a_{i j}, f_{i j}$, and $h_{i j}$ represent coefficients derived from vehicle mass/lift/drag properties, foil lift, and thruster properties respectively. Note the linear dependence on $U_{0}$ of the terms within $\boldsymbol{A}$, with the exception of the terms related to the metacentric restoring moment whose effect is invariant with speed. In contrast, the terms within $\boldsymbol{B}$ related to foil angle show a quadratic dependence on speed. The thruster terms on the right of the $\boldsymbol{B}$ matrix in the sway, roll, and yaw axes all cancel out if each port and starboard thruster pair are set to equal.

Numerical values for the constant coefficients in $\boldsymbol{A}$ and $\boldsymbol{B}$ were computed from vehicle parameter values and are given below in Tables 5.1 and 5.2.

\subsubsection{Decoupled Models}

The structure of the linearized system matrix $\boldsymbol{A}$ in (5.15) suggests that the system can be decoupled into two non-interacting subsystems:

1. $u, w, q, x, z, \theta$

2. $v, p, r, y, \phi, \psi$

This decomposition is further supported by the structure of the $\boldsymbol{B}$ matrix under the logical assumption that any thrust control action in the first subsystem is implemented 
Table 5.1: Near Horizontal Flight: Combined and Mass Coefficients

\begin{tabular}{cccc}
\hline \hline Coefficient & Value & Coefficient & Value \\
\hline$a_{11}$ & $-2.67 \mathrm{e}-02$ & $m_{11}$ & $4.44 \mathrm{e}-04$ \\
$a_{13}$ & $-3.76 \mathrm{e}-02$ & $m_{13}$ & $5.24 \mathrm{e}-06$ \\
$a_{15}$ & $5.94 \mathrm{e}-02$ & $m_{15}$ & $-5.70 \mathrm{e}-05$ \\
$a_{22}$ & $-4.73 \mathrm{e}-01$ & $m_{22}$ & $1.47 \mathrm{e}-04$ \\
$a_{24}$ & $-6.37 \mathrm{e}-02$ & $m_{24}$ & $5.58 \mathrm{e}-05$ \\
$a_{26}$ & $-4.32 \mathrm{e}-01$ & $m_{26}$ & $3.22 \mathrm{e}-05$ \\
$a_{31}$ & $-3.15 \mathrm{e}-04$ & $m_{31}$ & $5.24 \mathrm{e}-06$ \\
$a_{33}$ & $-5.09 \mathrm{e}-01$ & $m_{33}$ & $2.92 \mathrm{e}-04$ \\
$a_{35}$ & $5.66 \mathrm{e}-01$ & $m_{35}$ & $-3.13 \mathrm{e}-05$ \\
$a_{42}$ & $-2.35 \mathrm{e}-01$ & $m_{42}$ & $5.58 \mathrm{e}-05$ \\
$a_{44}$ & $-1.12 \mathrm{e}+00$ & $m_{44}$ & $9.79 \mathrm{e}-04$ \\
$a_{46}$ & $1.61 \mathrm{e}-01$ & $m_{46}$ & $2.44 \mathrm{e}-05$ \\
$a_{51}$ & $3.42 \mathrm{e}-03$ & $m_{51}$ & $-5.70 \mathrm{e}-05$ \\
$a_{53}$ & $2.25 \mathrm{e}-01$ & $m_{53}$ & $-3.13 \mathrm{e}-05$ \\
$a_{55}$ & $-3.55 \mathrm{e}-01$ & $m_{55}$ & $3.40 \mathrm{e}-04$ \\
$a_{62}$ & $-1.18 \mathrm{e}+00$ & $m_{62}$ & $3.22 \mathrm{e}-05$ \\
$a_{64}$ & $-2.78 \mathrm{e}-02$ & $m_{64}$ & $2.44 \mathrm{e}-05$ \\
$a_{66}$ & $-9.43 \mathrm{e}-01$ & $m_{66}$ & $2.46 \mathrm{e}-04$ \\
\hline \hline
\end{tabular}

Table 5.2: Near Horizontal Flight: Foil and Thruster Coefficients

\begin{tabular}{cccc}
\hline \hline Coefficient & Value & Coefficient & Value \\
\hline$f_{11}$ & $1.97 \mathrm{e}-02$ & $h_{11}$ & $2.71 \mathrm{e}-03$ \\
$f_{12}$ & $-2.45 \mathrm{e}-02$ & $h_{12}$ & $-3.53 \mathrm{e}-03$ \\
$f_{31}$ & $1.34 \mathrm{e}-01$ & $h_{31}$ & $1.89 \mathrm{e}-02$ \\
$f_{32}$ & $1.10 \mathrm{e}-01$ & $h_{32}$ & $1.54 \mathrm{e}-02$ \\
$f_{51}$ & $-1.18 \mathrm{e}-01$ & $h_{51}$ & $-1.62 \mathrm{e}-02$ \\
$f_{52}$ & $1.46 \mathrm{e}-01$ & $h_{52}$ & $2.11 \mathrm{e}-02$ \\
\hline \hline
\end{tabular}


using balanced thrust (i.e. $h_{f p}=h_{f s} ; h_{a p}=h_{a s}$ ), and any thrust control action in the second is implemented using differential thrust (i.e. $h_{f p}=-h_{f s} ; h_{a p}=-h_{a s}$ ). The symmetry of the latter four columns of $\boldsymbol{B}$ then ensures thrust inputs from control action in either subsystem will not interfere with the other. The foil inputs affect only the first subsystem as is evident from the first two columns of $\boldsymbol{B}$. 
Based on the coefficient values given in Tables 5.1 and 5.2, and following the approach in Healey and Lienard [7], we further propose to separate subsystems (1) and (2) into two pairs of lightly interacting subsystems:

1. speed: $u$

2. pitch-depth: $w, q, z, \theta$

3. heading: $v, r, \psi$

4. roll: $p, \phi$

We have ignored the states $x$ and $y$ since the Sentry vehicle is not directly actuated in sway, and its horizontal position will be controlled indirectly through heading and speed.

The speed state $u$ is coupled to the pitch-depth states $w, q, z, \theta$ primarily through pitch $\theta$ and pitch rate $q$, thus the decoupling suggested above is only valid under the assumption that the pitch and pitch rate will remain small. The static stability of the vehicle combined with the mission requirement that pitch rate remain small justify these assumptions, assuming that the latter can be satisfied by an acceptable control design. Aggressive control (i.e. large foil angles) will violate this assumption.

The coefficient values coupling the roll states $p$ and $\phi$ to the rest of subsystem (2) are at least an order of magnitude smaller than coefficients internal to subsystem (2). The converse is not true: the states in subsystem (3) exert some influence over the roll states; however the static stability of the vehicle combined with a low heading control bandwidth requirement suggest the roll states can be safely ignored in control design and left passive.

Under these assumptions, linear control design can be pursued independently on the three subsystems (1), (2), and (3) given by:

$$
\begin{aligned}
& \dot{u}=a_{11} U_{o} u+m_{11}\left(h_{f p}+h_{f s}+h_{a p}+h_{a s}\right) \\
& {\left[\begin{array}{c}
\dot{w} \\
\dot{q} \\
\dot{\dot{z}} \\
\dot{\theta}
\end{array}\right]=\left[\begin{array}{cccc}
a_{33} U_{o} & a_{35} U_{o} & 0 & -m_{35} z_{G B} W \\
a_{53} U_{o} & a_{55} U_{o} & 0 & -m_{55} z_{G B} W \\
1 & 0 & 0 & U_{o} \\
0 & 1 & 0 & 0
\end{array}\right]\left[\begin{array}{c}
w \\
q \\
z \\
\theta
\end{array}\right]+\left[\begin{array}{cc}
\frac{\beta_{h}}{1+\beta_{h}} h_{31}+U_{o}^{2} f_{31} & \frac{1}{1+\beta_{h}} h_{32}+U_{o}^{2} f 32 \\
\frac{\beta_{h}}{1+\beta_{h}} h_{51}+U_{o}^{2} f_{51} & \frac{1}{1+\beta_{h}} h_{52}+U_{o}^{2} f 52 \\
0 & 0 \\
0 & 0
\end{array}\right]\left[\begin{array}{c}
\phi_{f f} \\
\phi_{a f}
\end{array}\right]} \\
& {\left[\begin{array}{c}
\dot{v} \\
\dot{r} \\
\dot{\psi}
\end{array}\right]=\left[\begin{array}{ccc}
a_{22} U_{o} & a_{26} U_{o} & 0 \\
a_{62} U_{o} & a_{66} U_{o} & 0 \\
0 & 1 & 0
\end{array}\right]\left[\begin{array}{c}
v \\
r \\
\psi
\end{array}\right]+\left[\begin{array}{cccc}
m_{26} b_{h} & -m_{26} b_{h} & m_{26} b_{h} & -m_{26} b_{h} \\
m_{66} b_{h} & -m_{66} b_{h} & m_{66} b_{h} & -m_{66} b_{h} \\
0 & 0 & 0 & 0
\end{array}\right]\left[\begin{array}{c}
h_{f p} \\
h_{f s} \\
h_{\text {ap }} \\
h_{\text {as }}
\end{array}\right]}
\end{aligned}
$$

The roll states are left passive.

It is important to note that several second order, but potentially significant forms of input coupling are not evident in the linearized version of the input matrix $\boldsymbol{B}$ above. 
Specifically, reduction in forward thrust, decreased control over yaw, decreased foil control over pitch and coupling into the roll axis caused by non-zero foil angles are not represented. These are significant effects at high foil angles, and controller design based on these linearized models must take these effects into account. The coupling evident between axes in $B$ is due only to the inverse inertia matrix $M^{-}$; non-diagonal elements are due to the large CB/CG separation of the vehicle.

\subsection{Nonlinearity Considerations}

The linearized models presented in the previous section are all predicated on the assumption that the foils provide linearly increasing control effort as foil angle is increased. In this section we consider conditions for which this assumption proves invalid. Specifically, we discuss nonlinearity due to physical flow separation, and nonlinearity due to vehicle geometry. Particularly the latter phenomenon becomes problematic for flight at high angles of attack $\alpha$.

\subsubsection{Flow Separation: Foil Stall}

Our foil linearity assumption is physically invalid at high foil angles of attack because of flow separation and the resultant loss of lift. This condition is referred to as "stall." However, the foils are large enough relative to body size and mass that, assuming concerted foil motion (i.e. $\phi_{f f} \approx \phi_{a f}$ ) and sufficiently slow foil rotation, the angles of attack on each foil will remain small as the vehicle alters course due to the applied foil lift. Large foil angles imply large vertical velocity, but are not likely to violate the assumption of a linear lift slope.

Of course, under such conditions the hydrodynamics of the body change considerably. At sufficient angle of attack, the roots will stall, and vehicle drag in the direction of motion will increase markedly. The drag of the body also increases as angle of attack increases. Increased drag in turn affects vehicle speed and damping in all axes. The structure of the system matrix $\boldsymbol{A}$ remains invariant, but the magnitude of its components change.

\subsubsection{Input Mapping Nonlinearity}

Of far greater importance to vehicle control is the deviation from our linear nearhorizontal flight model caused by the change in input mappings as the foil angles increase. The effect is intuitive: at $\phi_{f}=90^{\circ}$, and for nearly vertical vehicle motion, 
a change in foil angle produces force only in the $x$-direction. Similarly, a change in thrust no longer directly affects the $x$-axis, and is instead mapped into the $z$ and pitch axes of the vehicle.

It is instructive to investigate these phenomena algebraically. Under the assumption of a linear lift slope it is straightforward to show that the torque in pitch generated by the foils is given by:

$$
\begin{aligned}
\tau_{\theta}=a_{f f}\left[L_{o}\left(\operatorname{atan}\left(\frac{w}{u}\right)-\phi_{f f}\right) \cos \left(\operatorname{atan}\left(\frac{w}{u}\right)\right)\left(u^{2}+w^{2}\right)\right] \\
+a_{a f}\left[L_{o}\left(\operatorname{atan}\left(\frac{w}{u}\right)-\phi_{a f}\right) \cos \left(\operatorname{atan}\left(\frac{w}{u}\right)\right)\left(u^{2}+w^{2}\right)\right] .
\end{aligned}
$$

The constant $L_{o}$ scales the lift generated per unit $\alpha$ appropriately.

In Chapter 6 we define a proportionality constant $\gamma_{f}=\frac{\phi_{f f}}{\phi_{a f}} \leq 0$ between the forward and aft foil angles that effectively recasts pitch control in terms of a single input variable $\phi_{a f_{\theta}}$ that controls the differential foil angle (see (6.7)). In terms of $\phi_{a f_{\theta}}$, equation (5.20) becomes

$$
\begin{aligned}
\tau_{\theta}=a_{f f}\left[L_{o}\left(\operatorname{atan}\left(\frac{w}{u}\right)-\gamma_{a} \phi_{a f_{\theta}}\right) \cos \left(\operatorname{atan}\left(\frac{w}{u}\right)\right)\left(u^{2}+w^{2}\right)\right] \\
+a_{a f}\left[L_{o}\left(\operatorname{atan}\left(\frac{w}{u}\right)-\phi_{a f_{\theta}}\right) \cos \left(\operatorname{atan}\left(\frac{w}{u}\right)\right)\left(u^{2}+w^{2}\right)\right] .
\end{aligned}
$$

We then define the physical control gain

$$
\frac{\delta \tau_{\theta}}{\delta \phi_{a f_{\theta}}}=L_{o} \cos \left(\operatorname{atan}\left(\frac{w}{u}\right)\right)\left(u^{2}+w^{2}\right)\left(a_{f f} \gamma_{f}+a_{a f}\right)
$$

The arctangent in the expression above is simply the vehicle angle of attack, thus the foil-to-pitch control gain can be expressed compactly as a linear function of vehicle angle of attack $\alpha$ alone:

$$
\frac{\delta \tau_{\theta}}{\delta \phi_{a f_{\theta}}}=C_{1} \cos \alpha
$$

where $C_{1}$ represents a leading coefficient dependent upon vehicle speed. It is then immediate that changes in foil angle near $\phi_{f}=90^{\circ}$ will not affect vehicle pitch. Conversely, at $\phi_{f}=0^{\circ}$ the foils exhibit a maximum in physical control over vehicle pitch.

At the same time, it is direct from the structure of the nonlinear input matrix (3.15) that thruster control over pitch increases to a maximum at $\phi_{f}=90^{\circ}$ :

$$
\frac{\delta \tau_{\theta}}{\delta h_{\theta}}=C_{2} \sin \phi_{f}
$$


where the forward/aft differential thrust $h_{\theta}$ is defined as:

$$
h_{\theta}=h_{f p}+h_{f s}-\left(h_{a p}+h_{a s}\right) .
$$

A similar argument can be applied to the thrusters with respect to control over vehicle yaw. Again proceeding directly from the nonlinear input matrix (3.15),

$$
\frac{\delta \tau_{\psi}}{\delta h_{\psi}}=C_{3} \cos \phi_{f}
$$

where the lateral differential thrust $h_{\psi}$ is defined as:

$$
h_{\psi}=h_{f p}-h_{f s}+h_{a p}-h_{a s}
$$

Unlike for the longitudinal axis where thrust control over longitudinal dynamics increases as foil control decreases, the yaw dynamics of the vehicle are dependent entirely upon the thrusters, thus for $\phi_{f}=90^{\circ}$ the vehicle is not directly actuated in yaw. 


\section{Chapter 6}

\section{Controller Design}

In this chapter we consider the design and analysis of a linear controller based on the linearized equations of motion for near-horizontal flight developed in Section 5.2. Various forms of linear control have been applied to many AUVs because of the simplicity and robustness of the resulting controller in addition to the wide variety of analysis techniques available to aid in the design process (e.g. $[5,15]$ ).

We apply the decoupled control design paradigm suggested by [5], to the nearhorizontal flight linearized model to which it is shown to be well suited. Simulation results on the full nonlinear model, as well as experimental results from an implementation of the resulting controller on a $1 / 4$-scale physical model (Appendix B are presented. The linear controller designed herein performs surprisingly well, even well outside of the range it was designed for, although control over pitch and yaw is severely degraded for large foil angles. Ultimately, however, the mapping non-linearity of the inputs (Section 5.3) restricts the linear controller from taking full advantage of the dynamic range made available by Sentry's unique design.

These shortcomings motivate a nonlinear controller, of which many forms have been applied to underwater robotic systems (cf. $[28,5,7,26]$ ), although typically with the goal of compensating for the non-linearity and uncertainty inherent in the hydrodynamics, rather than non-linearity in the input, which is of greater importance for Sentry. We close by briefly considering a potential route towards a controller capable of taking advantage of Sentry's dynamic range, but leave its completion to future work. 


\subsection{Control Objective}

Simply stated, the objective of controller design for the Sentry vehicle is to control the vehicle such that it can fulfill its mission: the execution a series of straight tracklines at a constant height above the seafloor. Maintenance of a constant altitude above the seafloor is crucial to ensure optimal sensor resolution and data quality. This requirement stands as the most important goal of the control system. The mid-ocean spreading centers where Sentry will conduct most of its survey work are characterized by very rough topography necessitating a depth control algorithm with high spatial bandwidth. That is, the vehicle should be able to track the rough contours of the bottom at a horizontal speed sufficient to ensure the survey area is covered before battery depletion.

In this work, we do not delve into the design of the bottom following algorithm, and instead assume that the desired altitude has been determined and translated into a desired depth. We further assume the desired depth trajectory is similar to that generated by ABE's threshold based bottom-following algorithm [25] and consists of constant desired depth segments with smoothed ramp transitions between them.

The mission requirement that the vehicle follow straight tracklines across the ocean floor is met with a simple heading servo, although more complex schemes are required in the presence of significant currents. In the following design, we leave forward speed control open loop.

\subsection{Decoupled Control Design}

In this section we describe the design of a linear control system for nominally horizontal flight. The controller is designed using the linear decoupled model developed in Section 5.2 and given in equations (5.17)-(5.19). The decoupled system consists of three lightly interacting subsystems:

1. speed: $u$

2. pitch-depth: $w, q, z, \theta$

3. heading: $v, r, \psi$

The roll axis $(p, \phi)$ is left passive. 


\subsubsection{Speed Control}

The decoupled speed system is described by

$$
\dot{u}=a_{11} U_{o} u+m_{11}\left(h_{f p}+h_{f s}+h_{a p}+h_{a s}\right) .
$$

Rather than seek to control the speed via feedback, we apply a simple open loop thrust command that balances forward drag such that the vehicle maintains $u \approx U_{o}$ :

$$
h_{f p}=h_{f s}=h_{a p}=h_{a s}=-a_{11} U_{o}^{2}
$$

This approach limits the degree to which vehicle speed, which is of lesser importance from a mission perspective, will interfere with pitch-depth control.

\subsubsection{Pitch-Depth Control}

The decoupled pitch-depth system is described by

$$
\left[\begin{array}{c}
\dot{w} \\
\dot{q} \\
\dot{z} \\
\dot{\theta}
\end{array}\right]=\left[\begin{array}{cccc}
a_{33} U_{o} & a_{35} U_{o} & 0 & -m_{35} z_{G B} W \\
a_{53} U_{o} & a_{55} U_{o} & 0 & -m_{55} z_{G B} W \\
1 & 0 & 0 & U_{o} \\
0 & 1 & 0 & 0
\end{array}\right]\left[\begin{array}{c}
w \\
q \\
z \\
\theta
\end{array}\right]+\left[\begin{array}{cc}
\frac{\beta_{h}}{1+\beta_{h}} h_{31}+U_{o}^{2} f_{31} & \frac{1}{1+\beta_{h}} h_{32}+U_{o}^{2} f 32 \\
\frac{\beta_{h}}{1+\beta_{h}} h_{51}+U_{o}^{2} f_{51} & \frac{1}{1+\beta_{h}} h_{52}+U_{o}^{2} f 52 \\
0 & 0 \\
0 & 0
\end{array}\right]\left[\begin{array}{c}
\phi_{f f} \\
\phi_{a f}
\end{array}\right] .
$$

Effective depth regulation requires an understanding of the coupled pitch-depth dynamics of the vehicle. We begin by considering the open loop stability of the system.

Physically, the shape of the streamlined body and foils induce two opposing moments of hydrodynamic origin. The Munk moment, whose appearance is predicted for inviscid flow, is destabilizing. Body and foil lift also contribute to the net hydrodynamic moment incident on the vehicle. Lift from the forward control foil and root induces a destabilizing moment, while lift from the aft control foil and root induces a stabilizing moment. Body lift acts aft of the vehicle center of mass (see Section 4.4) and is therefore stabilizing. These moments sum to produce a small net stabilizing moment whose magnitude increases with vehicle speed.

The hydrodynamic stabilizing moment is supplemented at low speeds by a hydrostatic restoring moment. The hydrostatic restoring moment is substantial due to the vehicle's large CB/CG separation, but its magnitude is invariant with speed.

The combined effect produces a vehicle increasingly stable in pitch as speed increases.

Because there are two inputs into this system, we have considerable flexibility in 


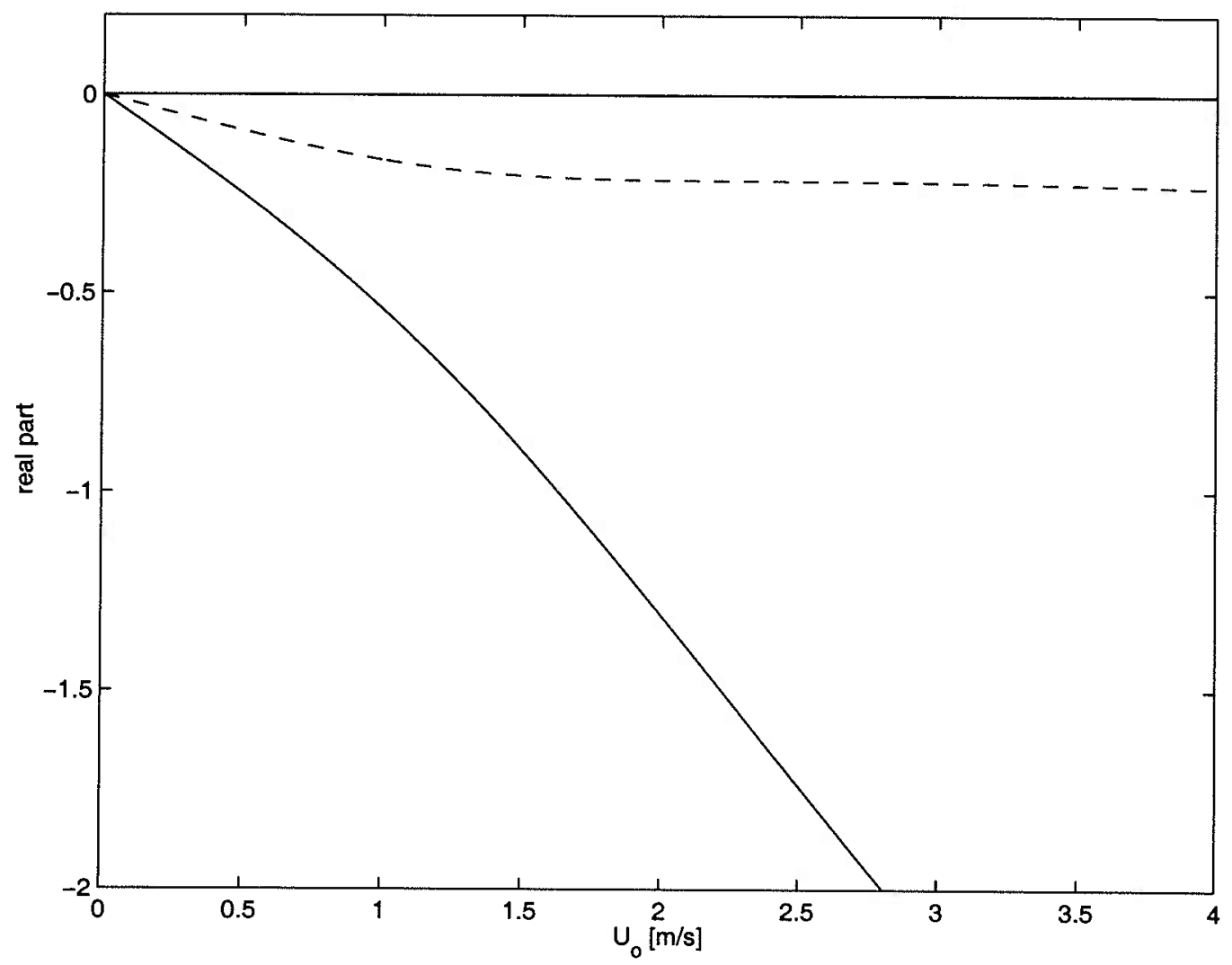

Figure 6-1: Eigenvalues of the open loop pitch-depth system as a function of nominal forward speed $U_{o}$. The dashed line represents the real parts of a pair of poles with nonzero imaginary parts. Note that the vehicle response is oscillatory over the entire speed range shown. The eigenvalues tend to the origin because low-speed linear drag terms, for which we have no estimate, have not been included.

determining the closed loop dynamics of the system. Intuitively, there are two basic means by which to effect a depth change:

1. Induce a non-zero pitch and wait for body lift and forward velocity to result in a depth change.

2. Change depth directly, without inducing pitch, by creating foil lift at both the fore and aft of the vehicle.

The first strategy is the means by which airplanes, submarines, and standard cigarshaped AUVs change depth. Their control surfaces are too small to create sufficient vertical force for a direct depth change. Sentry's foils are large compared to the body and can be used directly. However, changing depth while maintaining approximately zero pitch may be less efficient than strategy (1) because of the increased drag of the vehicle at non-zero angles of attack. 
The primary difference between (1) and (2) above is the relationship between the forward and aft foil control signals. Strategy (1) calls for either opposing foil angles, or no forward foil motion at all. Strategy (2) requires approximately equal foil angles for both the fore and aft foils. To investigate these two strategies we propose a change of input variables:

$$
\begin{aligned}
& \phi_{f f}=\phi_{f f_{z}}+\phi_{f f_{\theta}} \\
& \phi_{a f}=\phi_{a f_{z}}+\phi_{a f_{\theta}}
\end{aligned}
$$

and define the two ratios:

$$
\beta_{f}=\frac{\phi_{f f_{z}}}{\phi_{a f_{z}}} \quad \gamma_{f}=\frac{\phi_{f f_{\theta}}}{\phi_{a f_{\theta}}}
$$

The original inputs can be recovered by the transformation

$$
\left[\begin{array}{c}
\phi_{f f} \\
\phi_{a f}
\end{array}\right]=\left[\begin{array}{cc}
\beta_{f} & \gamma_{f} \\
1 & 1
\end{array}\right]\left[\begin{array}{l}
\phi_{a f_{z}} \\
\phi_{a f_{\theta}}
\end{array}\right] .
$$

The transformed inputs $\phi_{a f_{z}}, \phi_{a f_{\theta}}$ can be thought of as roughly independent inputs into the $\mathrm{z}$-axis and pitch-axis respectively. Thus, $\gamma_{f} \leq 0$ controls the differential gain between the two foils. The sign of $\beta_{f}$ determines whether the foils move together or opposite one another to effect a change in depth and its magnitude determines their ratio.

With this transformation of inputs, the input matrix $\boldsymbol{B}$ becomes

$$
\boldsymbol{B u}=\left[\begin{array}{cc}
b_{31} \beta_{f}+b_{32} & b_{31} \gamma_{f}+b_{32} \\
b_{51} \beta_{f}+b_{52} & b_{51} \gamma_{f}+b_{52} \\
0 & 0 \\
0 & 0
\end{array}\right]\left[\begin{array}{l}
\phi_{a f_{z}} \\
\phi_{a f_{\theta}}
\end{array}\right]
$$

where the expressions for $b_{i j}$ are dependent upon $U_{o}^{2}$ and are evident directly from (6.3). It is then immediate that setting

$$
\begin{aligned}
& \beta_{f}=\frac{-b_{52}}{b_{51}} \\
& \gamma_{f}=\frac{-b_{32}}{b_{31}}
\end{aligned}
$$

eliminates the direct coupling of $\phi_{a f_{z}}$ into the $\theta$-axis and of $\phi_{a f_{\theta}}$ into the $z$-axis. The axes are still coupled through the dynamics of the vehicle. As there is no reason to allow pitch control action to directly affect depth, we chose to set $\gamma_{f}$ as above in 
(6.10). The choice of $\beta_{f}$ is less obvious and depends on which depth change strategy is preferred.

Setting $\gamma_{f}=\frac{-b_{32}}{b_{31}}$ as suggested above yields

$$
\boldsymbol{B} \boldsymbol{u}=\left[\begin{array}{cc}
b_{31} \beta_{f}+b_{32} & 0 \\
b_{51} \beta_{f}+b_{52} & \frac{-b_{51} b_{32}}{b_{31}}+b_{52} \\
0 & 0 \\
0 & 0
\end{array}\right]\left[\begin{array}{l}
\phi_{a f_{\tilde{z}}} \\
\phi_{a f_{\theta}}
\end{array}\right]
$$

The transfer function between $\phi_{f f_{z}}$ and the depth $z$ is then

$$
\frac{z(s)}{\phi_{a f_{z}}(s)}=\frac{n_{2} s^{2}+n_{1} s+n_{0}}{|s \boldsymbol{I}-\boldsymbol{A}|}
$$

where

$$
\begin{aligned}
& n_{2}=c_{1} \\
& n_{1}=-\left(c_{1} a_{55} U_{o}+c_{2} U_{o}-c_{2} a_{35} U_{o}\right) \\
& n_{0}=-\left(c_{1} a_{53} U_{o}^{2}-c_{2} a_{33} U_{o}^{2}-c_{1} m_{55} z_{G} W+c_{2} m_{35} z_{G} W\right)
\end{aligned}
$$

and

$$
c_{1}=b_{31} \beta_{f}+b_{32} \quad c_{2}=b_{51} \beta_{f}+b_{52}
$$

The ratio $\beta_{f}$ affects only the denominator of 6.12 , but it determines both its sign and zero locations. Both these factors ultimately determine the vehicle response to a change in $\phi_{a f_{z}}$ (see Figure 6-2). 

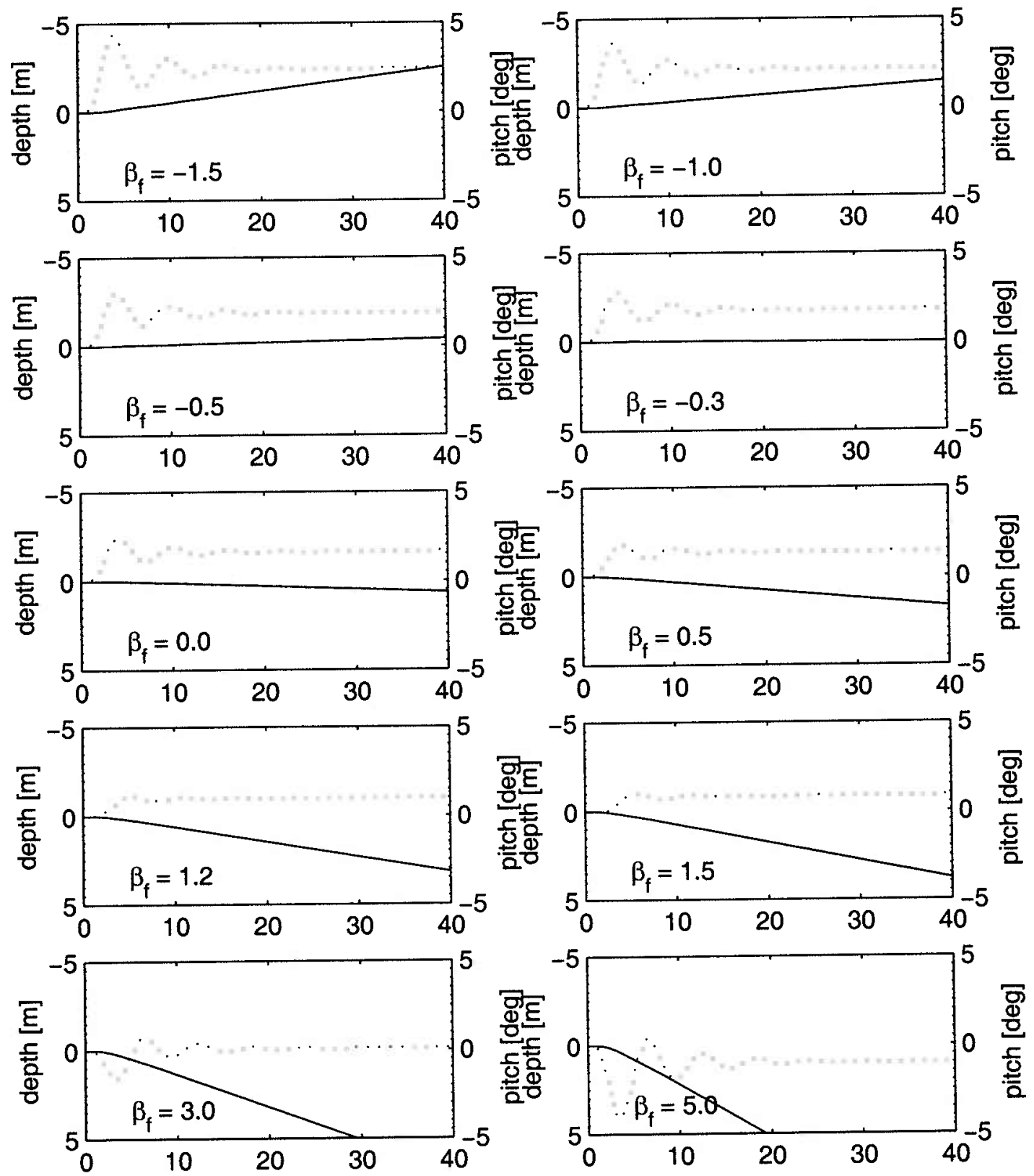

Figure 6-2: Vehicle Response (Linear Model) vs. $\beta_{f}$ at $U_{o}=1 \mathrm{~m} / \mathrm{s}$. The lowest two plots were produced at unrealistic $\beta_{f}$. 
The corresponding zero locations as a function of $\beta_{f}$ at $U_{0}=1 \mathrm{~m} / \mathrm{s}$ are shown in Figure 6-3. As is evident from the figures, the vehicle behaves in markedly different

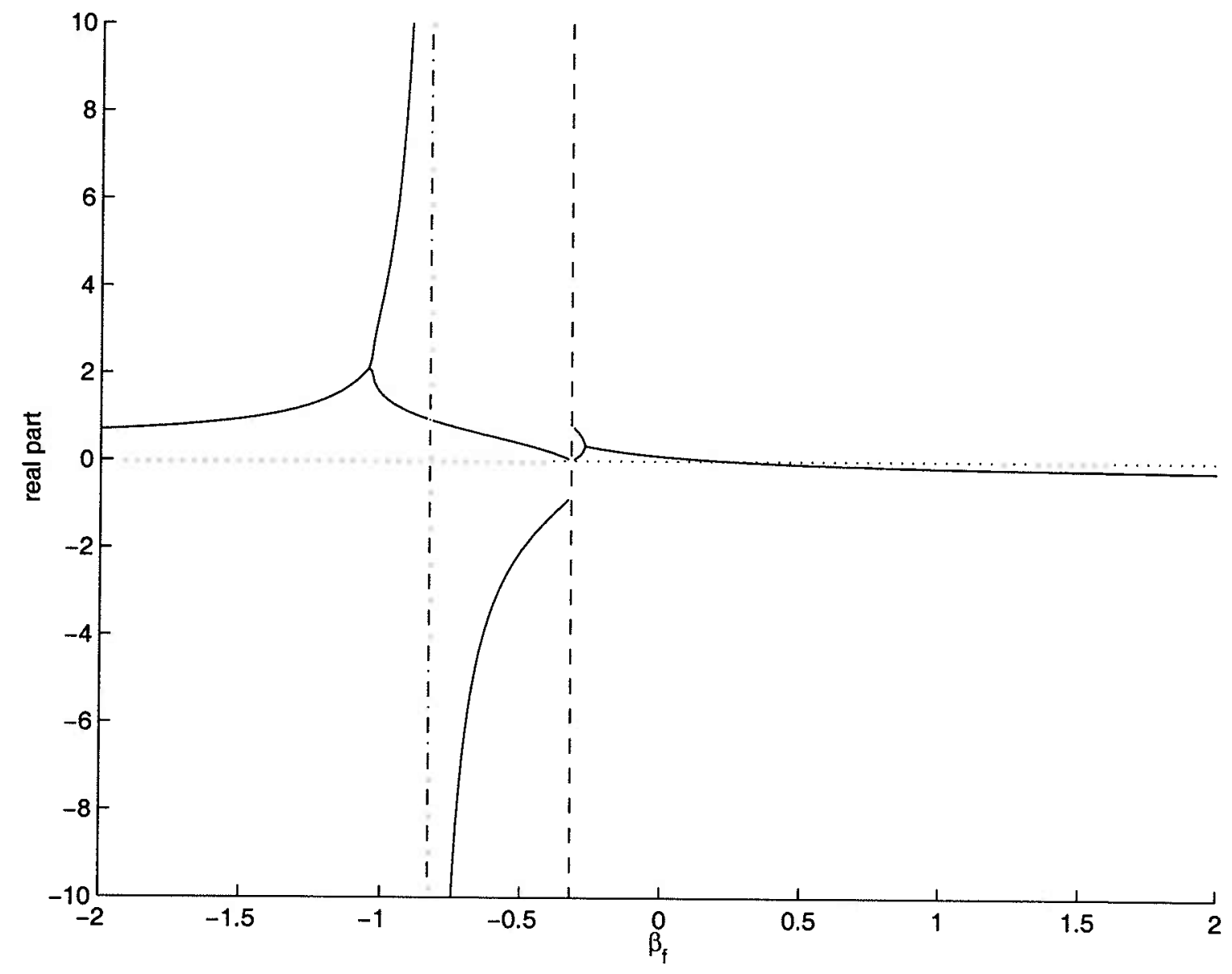

Figure 6-3: Real part of the open loop zeros as a function of $\beta_{f}$. To the right of the dashed line, the sign of the transfer function is positive; to the left it is negative. This line defines the division between depth change via strategy (1) (left of line) and strategy (2) (right of line).

ways over the range of practical $\beta_{f}$. Note in particular the sign change at $\beta_{f}=-0.3$ corresponding to a single zero at the origin. For $\beta_{f}<-0.3$, the vehicle changes depth via strategy (1). For $\beta_{f}>-0.3$, it changes depth via strategy (2). For small excursions in $\beta_{f}$ around this value, the system exhibits marked non-minimum phase behavior. As $\beta_{f}$ decreases, this behavior becomes less pronounced as the zeros become increasingly large. A reduction in order occurs at $\beta_{f}=\frac{-b_{32}}{b_{33}}$, corresponding to no direct coupling from the inputs to the z-axis. The bifurcation at slightly lesser $\beta_{f}$ effects the vehicle dynamics imperceptibly, as the zeros are too large compared to the dominant poles. 
As $\beta_{f}$ increases above -0.3 , a bifurcation is evident, beyond which the vehicle behaves in a modified non-minimum phase manner until the zeros cross into the left-half plane. Further increase in $\beta_{f}$ improves vehicle climb rate.

The pitch response of the vehicle is also of considerable importance and its characteristics likewise change as a function of $\beta_{f}$. Figure 6-4 shows the value of the single

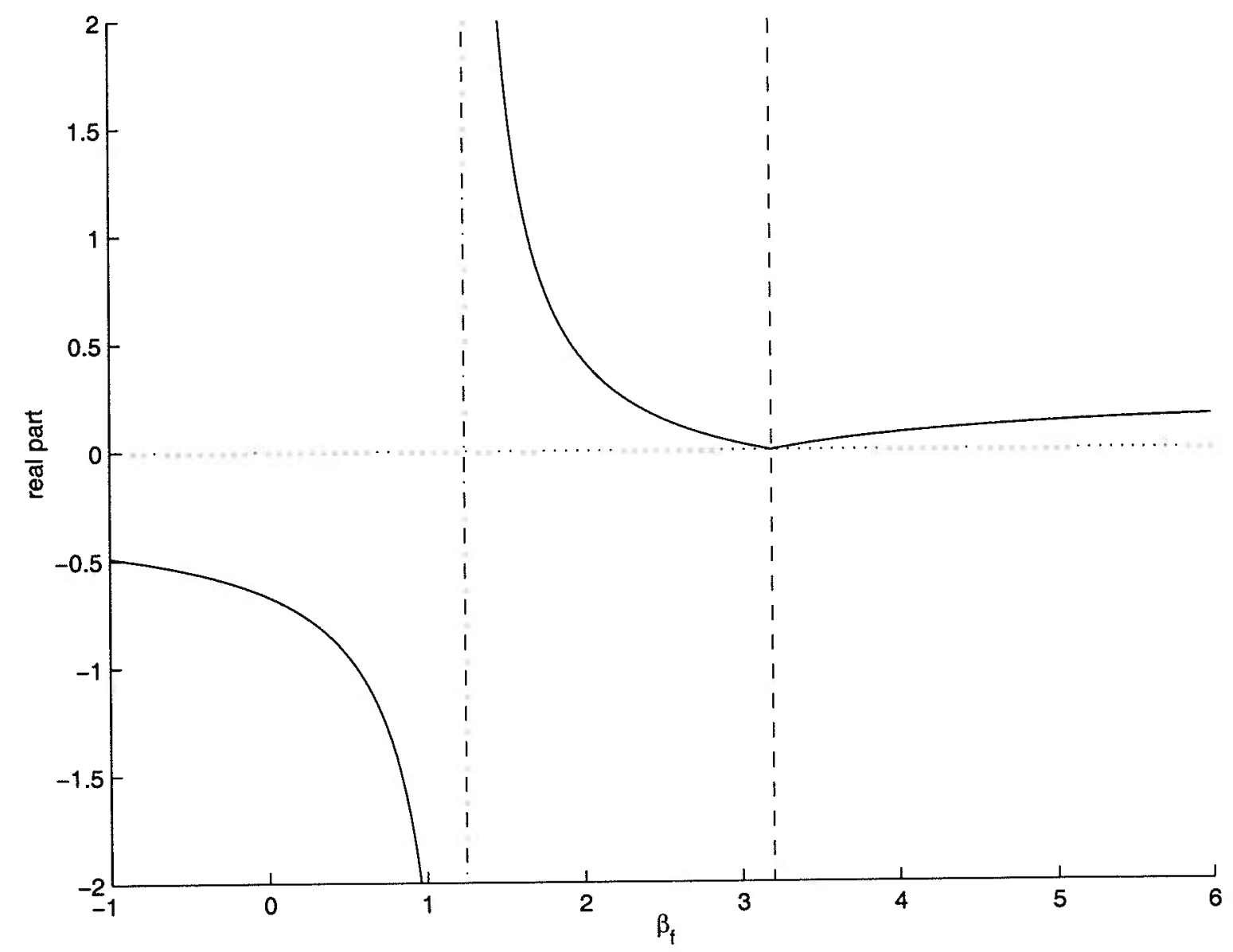

Figure 6-4: Zero of the open loop pitch-axis transfer function as a function of $\beta_{f}$. The dashed line corresponds to a sign change. To the right of this line, the sign of the transfer function is positive; to the left it is negative. Operation to the right of this line would result in more favorable steady state pitch; however, the magnitude of $\beta_{f}$ required implies unrealistic control foil angles.

zero of the transfer function between $\phi_{a f_{z}}$ and vehicle pitch:

$$
\frac{\theta(s)}{\phi_{a f_{z}}(s)}=\frac{c_{2} s^{2}+\left(c_{1} a_{53} U_{o}-c_{2} a_{33} U_{o}\right) s}{|s \boldsymbol{I}-\boldsymbol{A}|}
$$

where $c_{1}, c_{2}$, and $|s \boldsymbol{I}-\boldsymbol{A}|$ are as in equation (6.12). Note that the denominator 
is first order in the equation above as $s$ factors and cancels with the single pole at the origin. As is evident from the figure, a reduction of order occurs at $\beta_{f}=\frac{-b_{22}}{b_{21}}$ corresponding to no direct connection between $\phi_{a f_{z}}$ and vehicle pitch. Values of $\beta_{f}$ above this point result in non-minimum phase behavior.

For $-0.3 \leq \beta_{f} \leq 3.2$, only the pitch transfer function has a leading negative sign, implying the vehicle changes depth while pitched away from the direction of vertical motion. Physically this is the result of insufficient hydrodynamic stability to overcome the moment created by lift generated at the aft control foil (The moment created by the aft control foil overpowers that of the forward control foil because of its larger moment arm).

Below $\beta_{f}=-0.3$, the depth transfer function also becomes negative, and the vehicle pitches into the direction of vertical motion. The achievable steady state pitch; however, is limited at normal operating speed by the static stability of the vehicle, and thus the achievable climb rate is also limited.

Above $\beta_{f}=3.2$, the pitch transfer function changes sign, and the vehicle again pitches into the direction of vertical motion. However, such a large $\beta_{f}$ implies large foil angles thus violating our assumption of linearity and likely resulting in either physical stall or imposed saturation of the foils. Furthermore, the pitch response of the vehicle is undesirably oscillatory at large $\beta_{f}$.

From the preceding analysis and Figure $6-2, \beta_{f}$ must be below -0.3 to effect a depth change via strategy (1); However, the small steady state pitch achievable for realistic foil angles implies a limited climb rate and oscillatory pitch response. Thus, it is apparent that the Sentry vehicle is best suited to changing depth via strategy (2).

Physically, this results from the vehicle's large CB/CG separation, and relatively short body compared to foil size. The high degree of static stability requires a large control effort to alter pitch significantly, thus mitigating the effect of forward speed combined with steady pitch to alter depth. Concurrently, the short body length limits body lift generated from any nonzero pitch. Finally, the large foil areas mean that large forces are produced purely vertically, which, for insufficient vehicle pitch, dominate the body lift and pitched speed contribution to vertical velocity.

Unfortunately, the pitch response of the vehicle for reasonable $\beta_{f}$ is fairly uniformly oscillatory. It is at a minimum for $\beta_{f}=\frac{-b_{22}}{b_{21}}$ corresponding to no direct connection between $\phi_{a f_{z}}$ and vehicle pitch. As the nonminimum phase behavior for $\beta_{f}$ larger than this value is undesirable, we chose a conservatively lower value. From this point forward, we assume $\beta_{f}=1.2$. 
A more hydrodynamically stable vehicle would exhibit a more damped pitch response, as well as improved efficiency in a shallow climb.

We now move to the design of the closed depth regulator. We have seen that a minimal pitch response can be achieved open loop during a depth change by an appropriate choice of $\beta_{f}$. For this reason we seek only to damp the pitch rate. Low pitch rates are important from a mission perspective. We choose

$$
\phi_{a f_{\theta}}=-k_{D_{\theta}} q .
$$

where $k_{D_{\theta}} \geq 0$.

For the depth control law we chose a traditional proportional-integral-derivative (PID) controller:

$$
\phi_{a f_{z}}=k_{P_{z}}\left(z_{d}-z\right)-k_{D_{z}} w+k_{I_{z}} \int_{0}^{t}\left(z_{d}-z\right) d \tau .
$$

where the integral term should be limited to an imposed saturation value. Integral action has been added to assure bounded steady state error for ramp inputs of the type employed in ABE's bottom following algorithm. Because the foils are subject to the mapping non-linearity, imposed saturation limits, and stall, the integral term must be limited to avoid instabilities associated with integral windup.

The closed loop system is then

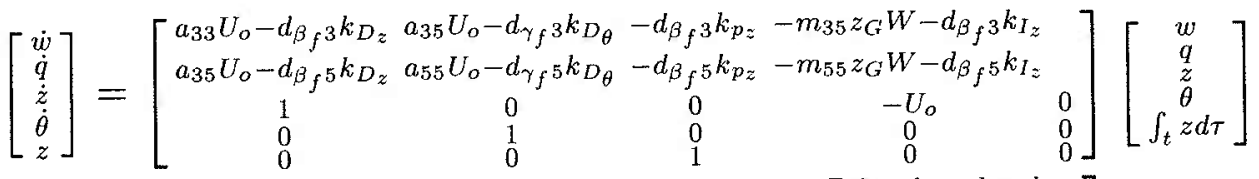

$$
\begin{aligned}
& +\left[\begin{array}{ccc}
d_{\beta_{\beta_{3}}} k_{p_{2}} & d_{\beta_{f^{3}}} k_{I_{z}} \\
d_{\beta_{f}} k_{p_{z}} & d_{\beta_{f}} k_{I_{z}} \\
0 & 0 \\
0 & 0 \\
0 & 0
\end{array}\right]\left[\begin{array}{c}
z_{d} \\
0
\end{array}\right]
\end{aligned}
$$

where

$$
\begin{array}{r}
d_{\phi_{f} i}=\left(\frac{\beta_{h}}{1+\beta_{h}} h_{i 1}+f_{i 1} U_{o}^{2}\right) \phi_{f}+\frac{1}{1+\beta_{h}} h_{i 2}+f_{i 2} U_{o}^{2} \\
\text { for } \quad i=\{3,5\}, \quad \phi_{f}=\left\{\beta_{f}, \gamma_{f}\right\}
\end{array}
$$

The gains, $k_{P_{z}}, k_{D_{z}}, k_{I_{z}}$ and $k_{D_{\theta}}$ should be chosen to satisfy the bandwidth requirements of the depth servo while taking care to avoid signals large enough to violate linearity. 


\subsubsection{Heading Control}

The decoupled heading system is described by

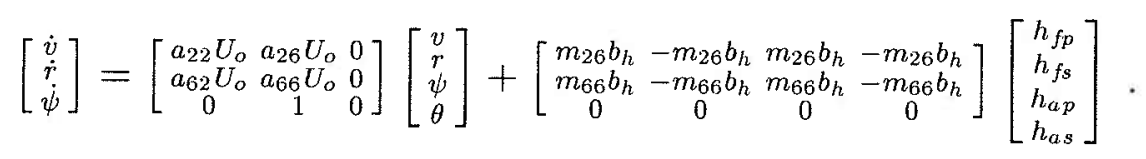

As with the pitch-depth system of the previous section, the open-loop stability of the heading system is dependent upon forward speed $U_{o}$, becoming increasingly unstable as $U_{o}$ increases. To see this, consider the characteristic equation of the system matrix in (6.18):

$$
\left|s \boldsymbol{I}-\boldsymbol{A}_{\psi}\right|=s^{2}-\left(a_{66}+a_{22}\right) U_{o} s+\left(a_{22} a_{66}-a_{26} a_{62}\right) U_{o}^{2} .
$$

Assuming $U_{o}>0$ a necessary and sufficient condition for system stability is given by

$$
\begin{gathered}
a_{22}+a_{66}<0 \\
a_{22} a_{66}-a_{26} a_{62}>0 .
\end{gathered}
$$

The first condition for stability is always met, for any vehicle design. The second depends closely on the magnitude and sign of $N_{v}$ [21], and is not satisfied in this case. Although $U_{o}$ does not render the vehicle stable or unstable, it is apparent from 6.19 that the vehicle will become less stable as speed increases.

Open loop instability in yaw is an undesirable system trait for the Sentry vehicle because stabilizing the vehicle will require constant control effort. Instability may be desired in systems that must be highly maneuverable; however, Sentry's mission profile calls for a series of long straight tracklines and thus a minimal heading bandwidth.

Heading instability does not effect the structure of the heading control system design that follows; however, it does necessitate sufficient gain to overcome the innate instability of the vehicle when operating at maximum speed.

Vehicle heading will be controlled by differential thrust and we introduce the change of input variables:

$$
h_{\psi}=\left(h_{f p}+h_{a p}\right)-\left(h_{f s}+h_{a s}\right) .
$$


The individual commanded thrusts can be recovered as follows:

$$
\begin{gathered}
h_{f p}=h_{a p}=\frac{1}{4} h_{\psi} \\
h_{f s}=h_{a s}=-\frac{1}{4} h_{\psi}
\end{gathered}
$$

where we have split the control effort equally among all four thrusters.

With this transformation of the inputs, the input matrix $\boldsymbol{B}_{\psi}$ becomes:

$$
\boldsymbol{B}_{\psi} \boldsymbol{u}=\left[\begin{array}{c}
m_{26} b_{h} \\
m_{6} b_{h} \\
0
\end{array}\right] h_{\psi} .
$$

The transfer function between the transformed input $h_{\psi}$ and heading is then:

$$
\frac{\psi(s)}{h_{\psi}(s)}=\frac{b_{h} m_{66} s+b_{h}\left(a_{62} m_{26}-a_{22} m_{66}\right) U_{o}}{s\left(s^{2}-\left(a_{22}+a_{66}\right) U_{o}+\left(a_{26} a_{66}-a_{26} a_{62}\right) U_{o}^{2}\right)} .
$$

Note that $m_{26} b_{h}$ is positive, but very small and thus this transfer function is minimum phase for all $U_{o}$. Unlike vehicles with a rudder that typically exhibit non-minimum phase behavior, no lateral force is produced by the thrusters when effecting a heading change, thus the only coupling into the sway axis is through $I_{x z}$. Despite Sentry's relatively large CB/CG separation compared to other AUVs, $I_{x z}$ is still small compared to the diagonal terms of $\boldsymbol{I}_{o}$

Based on the above analysis of the open loop dynamics, we propose a simple proportional-derivative (PD) heading controller of the form

$$
h_{\psi}=k_{P_{\psi}}\left(\psi_{d}-\psi\right)-k_{D_{r}} r
$$

The yaw rate $r$ and heading $\psi$ are readily available from the vehicle heading rate gyro and compass respectively. Integral action can be added to compensate for steady disturbances if necessary.

The closed loop dynamics is then

$$
\left[\begin{array}{c}
\dot{v} \\
\dot{r} \\
\dot{\psi}
\end{array}\right]=\left[\begin{array}{ccc}
a_{22} U_{o} & a_{26} U_{o}-k_{d_{r}} m_{26} b_{h} & -k_{p_{\psi}} m_{26} b_{h} \\
a_{62} U_{o} & a_{66} U_{o}-k_{d_{r}} m_{66} b_{h} & -k_{p_{\psi}} m_{66} b_{h} \\
0 & 1 & 0
\end{array}\right]\left[\begin{array}{c}
v \\
r \\
\psi
\end{array}\right]+\left[\begin{array}{c}
k_{p_{\psi}} m_{26} b_{h} \\
k_{p_{\psi}} m_{66} b_{h} \\
0
\end{array}\right] \psi_{d}
$$

where gains $k_{P_{\psi}}$ and $k_{D_{r}}$ must be chosen large enough to overcome the open loop instability of the vehicle. 


\subsection{Controller Evaluation: Simulation and Exper- imental Results}

In this section we present an analysis of controller performance from simulations conducted using the nonlinear vehicle model developed in Chapters 3 through 4 , and from controller implementation on a $\frac{1}{4}$-scale physical model of the vehicle (see Appendix B. The validity of this analysis is dependent upon the fidelity of both models. Since the derived parameters of the analytical model are likely to require adjustment once the full-scale vehicle becomes available, we focus here on behavioral trends which depend more on model structure than parameter values and control gains. Likewise, the lower Reynolds number and influence of the physical model's tether necessitate a qualitative interpretation of experimental results.

We begin by verifying the controller at the designed operating condition, both in simulation and using the physical model. That is, we define a smoothed ramped transition between two steady desired depths such that the linear vehicle model used in control design remains approximately valid. With the appropriate choices of control gains, the controller performs well both in simulation and implemented on the physical model.

The operating range over which the vehicle can be considered to behave in a linear fashion is qualitatively small. The hydrodynamics and rigid body dynamics of the vehicle are nonlinear, as are its inputs which vary nonlinearly with foil angle. Controller behavior in regimes where these nonlinearities are significant is interesting both in highlighting the shortcomings of the controller and pointing the way for future control development.

We will investigate two nonlinear effects: speed dependent performance and high angle-of-attack flight. Both these effects have been noted in previous chapters, particularly Chapter 5, and we will rely on that analysis to explain simulated vehicle behavior. Sensor limitations did not allow the investigation of these phenomena on the physical model.

\subsubsection{Controller Performance at Nominal Operating Condi- tions}

We begin by verifying controller performance at the designed operating condition: near constant forward speed $u \approx 1 \mathrm{~m} / \mathrm{s}$, small foil angles, and small vertical velocity.

To fulfill its mission, the vehicle must be able to handle considerably steeper 
climbs than those considered here, but steeper slopes imply significant nonlinearities unaccounted for in the linear control design. These nonlinearities and our controller's performance under their influence are treated later in this chapter.

\section{Simulation Results}

The simulated vehicle response shown in Figure 6-5 is to a ramp-smoothed transition between desired depths. The slope of the ramp was chosen to be shallow at $5^{\circ}$ (vs. time) to ensure the preceding conditions of approximate linearity were met.
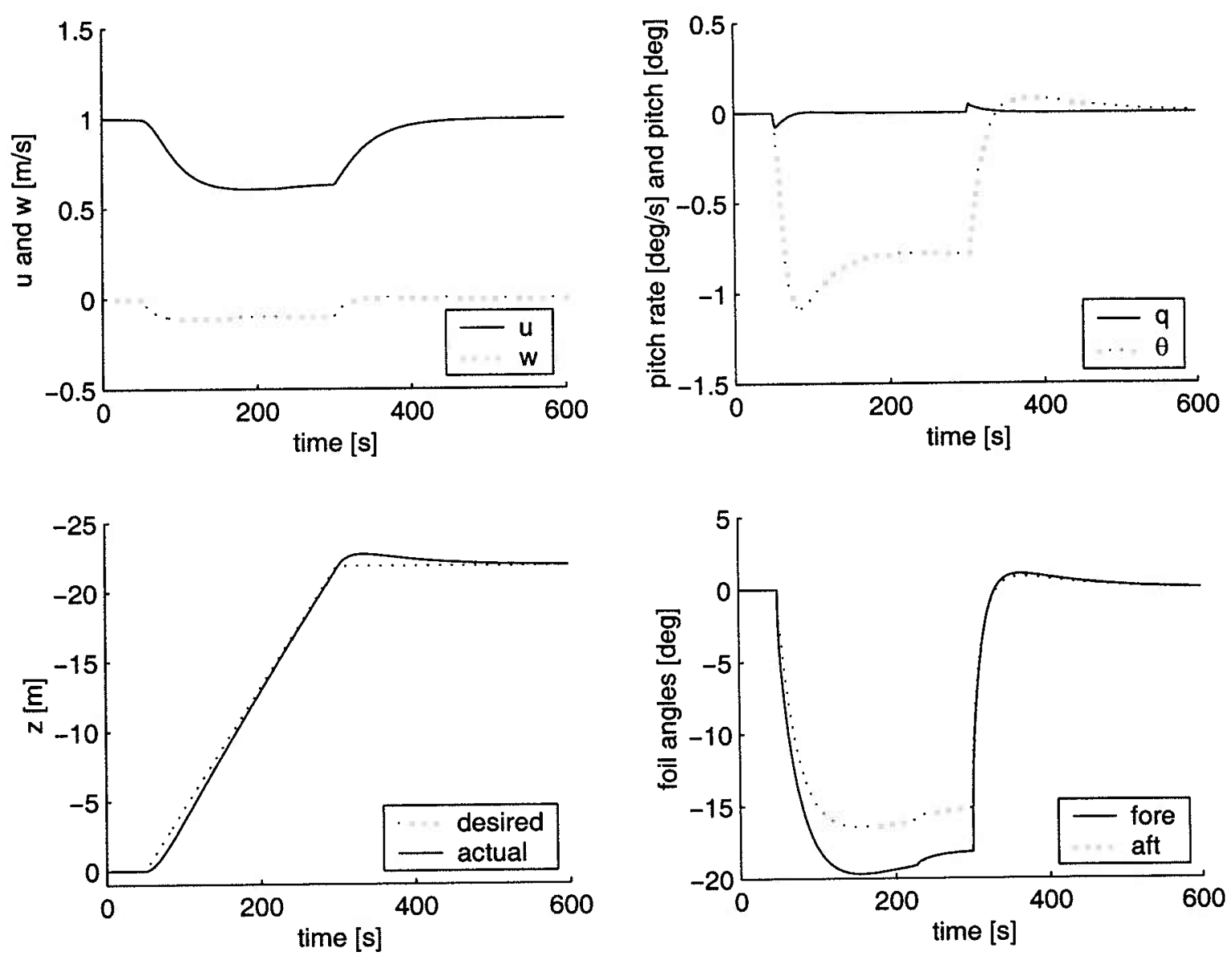

Figure 6-5: Vehicle Response to a Ramp-Smoothed Transition in Desired Depths.

The plots in Figure 6-5 were generated with control gains set to $k_{P_{z}}=1.0, k_{D_{z}}=$ $1.0, k_{I_{z}}=0.01$, and $k_{D_{\theta}}=10$. The depth change is achieved successfully with a maximum error of $0.37 \mathrm{~m}$. During the ramp portion of the depth change, forward speed is reduced due to increased vehicle drag and decreased thrust in the forward direction. The foil angles remain small: $18^{\circ}$ and $15^{\circ} \max$ for the fore and aft foils 
respectively.

The pitch response is well damped for this type of desired trajectory. A slight negative pitch accompanies the upward climb due to the larger moment arm of the aft foil relative to the forward foil.

The reduced forward speed of the vehicle means that the climb when viewed versus horizontal position rather than time becomes a slightly steeper ramp, ascending at $7.4^{\circ}$.

\section{Experimental Results}

We implemented the linear depth controller on the $\frac{1}{4}$-scale physical model and demonstrated its performance running at $10 \mathrm{Hzin}$ the flume facility at WHOI.

The vehicle was commanded to follow a repeating set ramp-smoothed $0.25 \mathrm{~m}$ depth changes while repeatedly running the length of the flume ( $\sim 12 \mathrm{~m}$ usual length). The model was controlled yaw by the author using a joystick and turned around in place at either end of each traverse without disengaging the depth controller. The commanded ramp slope was set to $50 \mathrm{~mm} / \mathrm{s}$. During traverses, vehicle speed was roughly constant at $0.4 \mathrm{~m} / \mathrm{s}$. This speed corresponds to a spatial climb angle of $7^{\circ}$.

The results for controller gains set to $k_{P_{z}}=1.0, k_{I_{z}}=0.1$, and $k_{D_{\theta}}=1$ are shown in Figure 6-6. The figure indicates the controller performs adequately. Grey shaded areas indicate turns, defined by a high sustained yaw rate (lowest plot). Foil control over depth deteriorates during turns when the forward speed drops to near zero. The shallow depth of the flume $\sim 1 \mathrm{~m}$ precluded an investigation as to whether the lag evident during climbs could be reduced by an increase in $k_{I_{z}}$. 

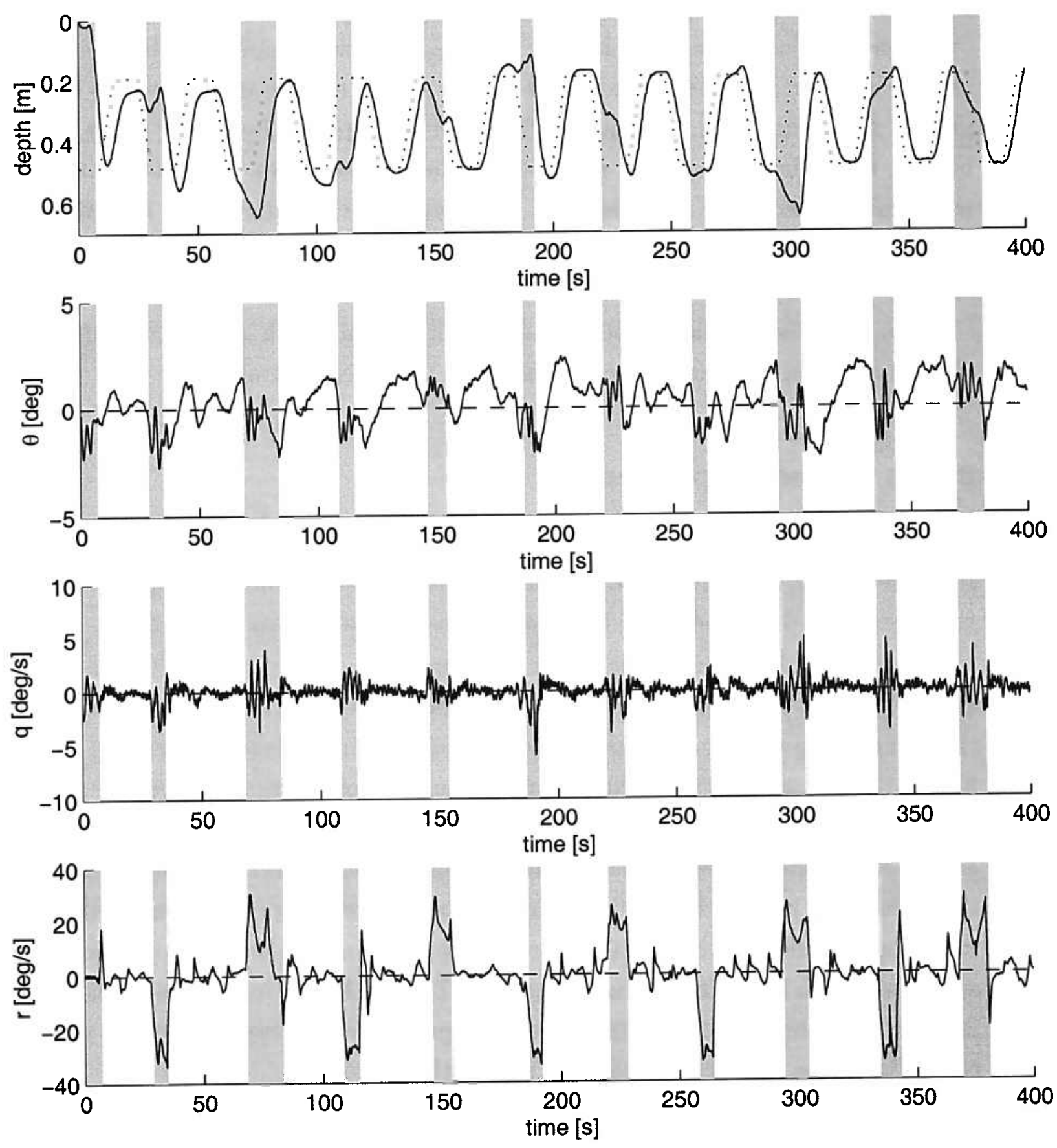

Figure 6-6: Controller Performance as Implemented on the Physical Model. The shaded grey areas indicate $180^{\circ}$ turns in place. 
Vehicle pitch versus time shown in the second plot does not show a strong correlation between pitch angle and vehicle angle of attack as predicted. This may be due to the forces generated at the stern of the vehicle while dragging the tether.

Pitch rate derivative gains ranging $0 \leq k_{D_{\theta}} \leq 2$ were applied with inconclusive results; however, it was evident that pitch was well damped during traverses in comparison to during turns without significant forward speed. For $k_{D_{\theta}} \geq 3$ the vehicle became unstable in pitch as a result of unmodeled dynamics associated with rotational foil accelerations and velocities. The magnitude of these forces and moments generated are disproportionatly large for the physical model due to the large relative size and weight of the thruster housings.

Note that the physical model does not provide a depth rate $w$ measurement. Depth rate damping was not implemented for these demonstrations.

\subsubsection{Speed Dependence}

In this section, we investigate the effect of increased vehicle speed on pitch and depth response by analyzing simulated vehicle response to a step in desired depth. We show the closed-loop depth behavior becomes increasingly stiff as speed increases.

Figure 6-7 shows the vehicle pitch and depth response to a $1 \mathrm{~m}$ step change in desired depth as a function of time for three different nominal forward speeds covering the vehicle's operating envelope. The controller gains selected were: $k_{P_{z}}=1.0$; $k_{D_{z}}=1.0 ; k_{I_{z}}=0.01 ; k_{D_{\theta}}$. Note that the control foils were not modeled as ratelimited for these simulations. The vehicle depth response shows the controller works adequately at speeds away from its $1 \mathrm{~m} / \mathrm{s}$ design speed. However, from Figure 6-7 it is evident that as vehicle speed increases, vehicle depth response becomes becomes increasingly stiffer. Vehicle pitch response becomes more damped with increasing speed, although the initial excursion grows. The forth plot in the figure shows the foil effort required decreases with increasing speed after the initial response which is equal due to the proportional term of the depth controller.

Physically, the increasing stiffness, reduced control effort, and improved pitch damping result from increased foil lift at higher fluid velocity. Recall from Section 5.2 that the linearized model predicted a quadratic increase in foil control gain, while predicting the vehicle dynamics would be only linearly dependent on speed. The larger nominal thrust required at higher nominal velocities also increases the effective foil gain, markedly at large foil angles, but only linearly at small foil angles. Thus, increased vehicle speed effectively increases control gain, although at the cost of higher 

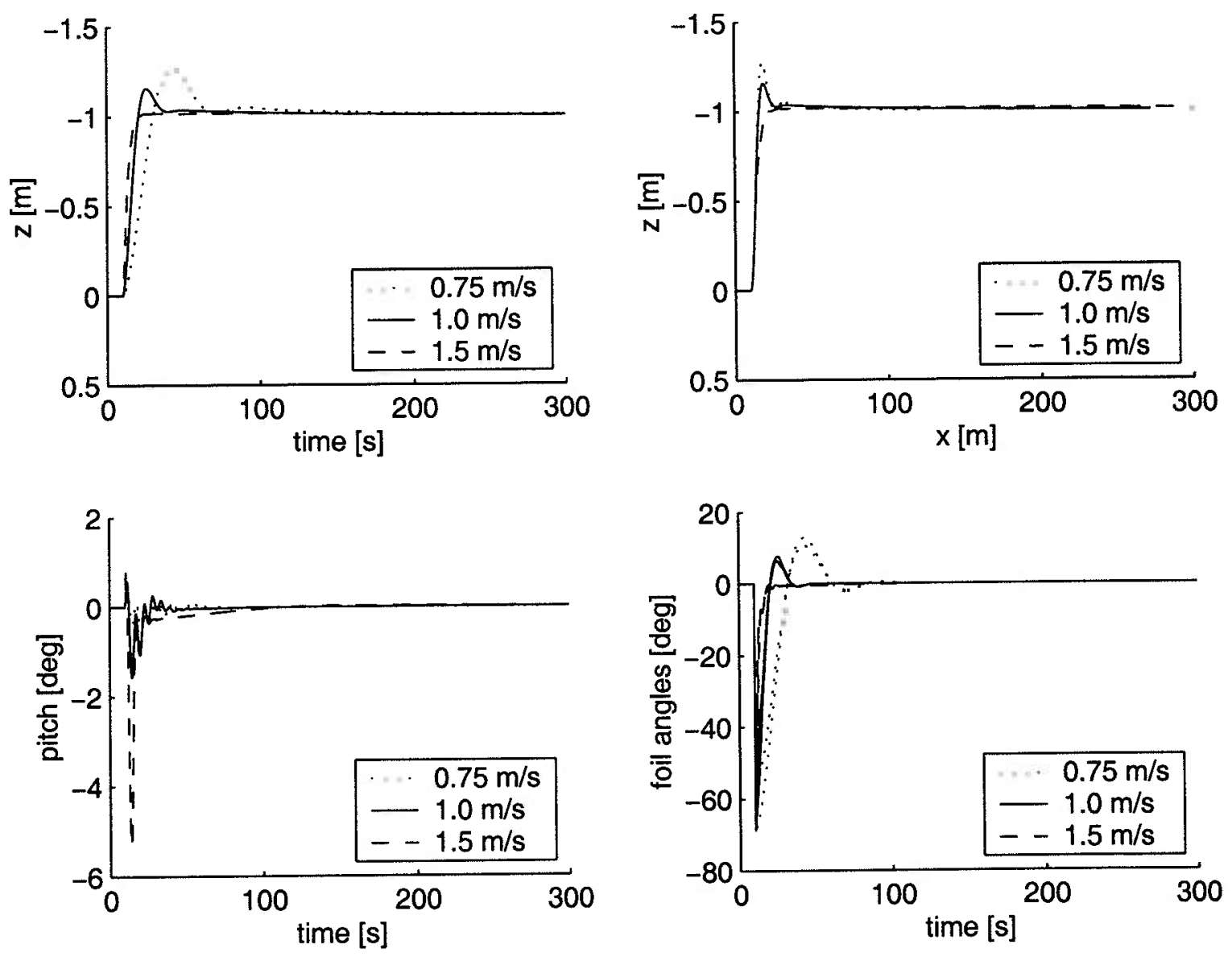

Figure 6-7: Vehicle pitch and depth response to a $1 \mathrm{~m}$ step change in desired depth for $U_{o}=0.5 \mathrm{~m} / \mathrm{s}, 1.0 \mathrm{~m} / \mathrm{s}$, and $2.0 \mathrm{~m} / \mathrm{s}$. The second plot of depth vs. horizontal distance $x$ has been shifted such that the change in commanded depth appears to occur at the same spatial location for comparison.

nominal thruster settings.

For all speeds simulated, the pitch response of the vehicle is undesirably oscillatory. Physically, this is the result of the severe negative acceleration caused by the increased drag of the foils reacting to the step depth error. The momentum of the vehicle's center of mass reacts to drag force applied at the vehicle centerline by swinging upwards, which sets up a slowly decaying oscillation. The decay is considerably longer at slow speeds where the effect of the pitch damper is mitigated.

To reduce the magnitude of the pitch oscillations, one possibility would be to increase the pitch damping gain $k_{D_{\theta}}$; however, this comes at the cost of excessively large foil angles and all the ensuing saturation problems. Indeed, for the plots shown in Figure $6-7, k_{D_{\theta}}$ was set deliberately low to avoid wild foil angle fluctuations. Rather, 
the oscillatory pitch response should be viewed as an argument against the appropriateness of step changes in desired depth as inputs. All but the smallest steps (relative to $k_{P_{z}}$ ) will result in large foil angles, severe reductions in speed due to increased drag, and the ensuing oscillations in pitch. Step inputs are, however, useful to illustrate vehicle behaviors.

Finally, note that when viewed versus horizontal distance travelled rather than time, the vehicle reaches near steady state in approximately the same horizontal distance, although the low-speed response is still considerably more oscillatory.

For these simulations, only a three degree-of-freedom model $(x, z, \theta)$ was used.

\subsubsection{Controller Performance at High Angles of Attack}

The linear controller performs reasonably at high angles of attack where the linearized vehicle model used to develop it no longer applies; however, control over pitch and yaw are compromised. Pitch damping is reduced, although the system remains stable in pitch. In the presence of a yaw disturbance, the inherent instability of the vehicle in yaw ensures the controller will fail.

Although the depth controller proposed no longer has a physical basis, the controller reacts reasonably to large errors in depth so long as the foil angles are clipped to $\pm 90^{\circ}$. That is, the proportional and integral terms work to point the foils towards the desired depth which works because the vehicle travels more or less parallel to its foils. At higher velocities, this is a consequence foils' large size relative to the foil. At lower velocities, this still holds true because the thrusters are mounted on the rotating control foils.

The pitch damper and yaw controller do not work well at high angles of attack. As discussed in Section 5.3, a change in foil angle no longer effects pitch, and likewise differential thrust couples into roll rather than yaw.

To illustrate controller behavior at high angles of attack, the vehicle was simulated following an increasingly steep set of ramped pulses while under the influence of sinusoidal disturbance torques in pitch and yaw. Figure 6-8 shows the vehicle response for a pitch disturbance of magnitude $2 \mathrm{~N} \cdot \mathrm{m}$ and frequency $0.18 \mathrm{~Hz}$. Note that $0.18 \mathrm{~Hz}$ is the vehicle's natural pitching frequency (in the linear approximation). Figure 6-9 shows the detrimental effect of the yaw controller at high angles of attack for a yaw disturbance of magnitude $0.1 \mathrm{~N} \cdot \mathrm{m}$ and frequency $0.01 \mathrm{~Hz}$. All plots were generated using controller gains $k_{P_{z}}=1, k_{D_{z}}=10, k_{I_{z}}=0.01, k_{D_{\theta}}=10, k_{P_{\psi}}=1000$, and $k_{D_{\psi}}=10$. A very stiff proportional term in the yaw controller was required to 
stabilize the vehicle laterally.
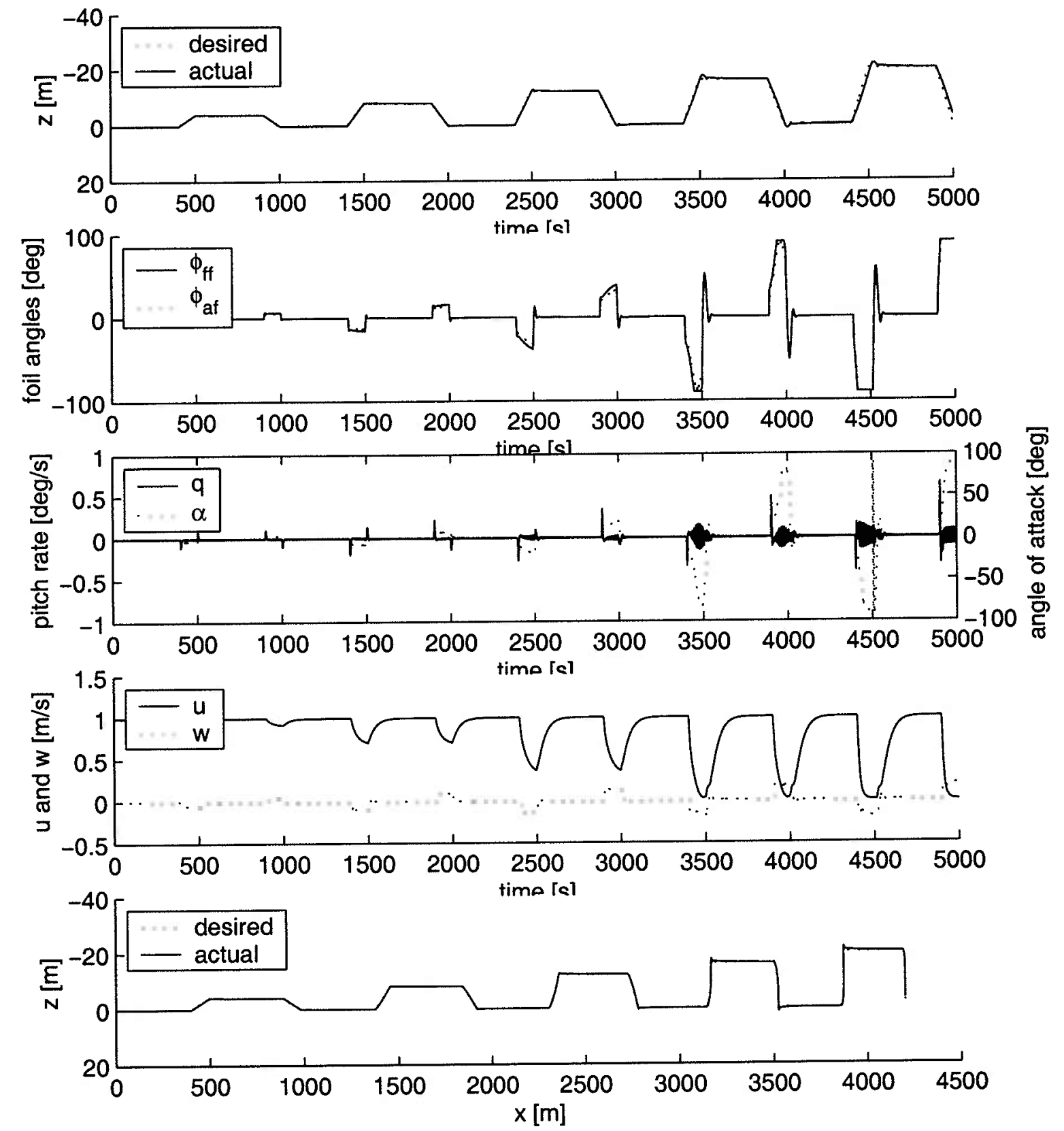

Figure 6-8: Simulated Vehicle Response with Pitch Disturbance.

Figure 6-8 demonstrates the vehicle successfully tracks spatial slopes approaching $90^{\circ}$ by saturating the control foils. These conditions are well outside the linear range used to design the controller.

Depth controller performance, even in the absence of pitch and yaw disturbances, is not ideal. Steeper ramps could be followed by increasing thrust at high angle of 


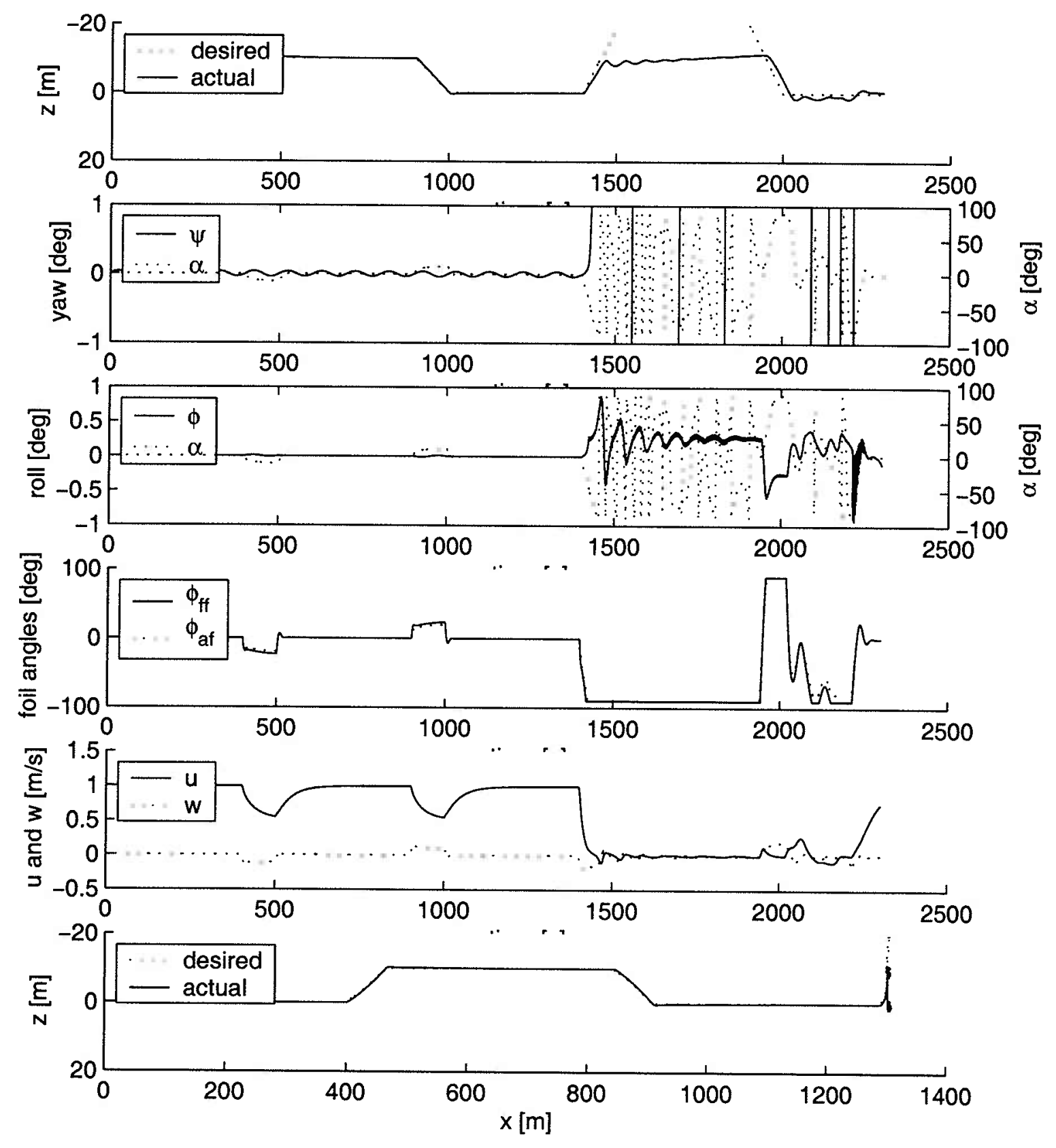

Figure 6-9: Simulated Vehicle Response with Yaw Disturbance.

attack to compensate for the increased drag. The depth controller as designed only maps to the foils, and works at high foil angles serendipitously rather than by design since foil lift no longer acts in the vertical axis.

This change of input mappings severly degrades pitch damping and yaw control. This is evident from the lower plot in Figure 6-8 which shows a marked increase in forced pitch rate oscillations for high vehicle angle of attack, or equivalently for large 
foil angles.

Similarly, Figure 6-9 shows an increase in yaw oscillations and roll disturbances during climbs requiring large foil angles. Furthermore, for steep enough climbs of sufficient duration the yaw controller interferes with the depth controller to the extent that the vehicle no longer tracks desired depth. The lateral instability of the vehicle ensures that any small disturbance in yaw becomes larger, and the resulting response in differential thrust sets up a slow oscillation in roll which in turn transforms the differential thrust into lateral force. The drag of the vehicle's tail transforms this lateral force into the spin shown at the right end of the bottom plot. The vehicle continues to rise during this spin, but at a severly reduced rate.

At high angle of attack, active thruster control could be implemented to damp pitch. For high $\alpha$, the foil-based pitch damper no longer has any effect. Yaw control is more troublesome in the sense that not only is there no replacement for differential thrust control over yaw, but as the foil angles increase, differential thrust couples instead into vehicle roll, which from a mission perspective is as detrimental as pitch disturbances.

The fact that effective yaw control is limited to small foil angles makes lateral stability crucial to performance. Had the forcing in yaw been of greater magnitude, the vehicle would have become unstable at smaller foil angles. Enough vertical foil area should be added to the rear of the vehicle to ensure lateral stability. Even so, for near-vertical flight yaw must either be left uncontrolled, or the vehicle must switch to an alternate foil configuration to maintain control over yaw.

The physical model has been used by the author in its present form and in an earlier form by Serfas [19] to explore various foils-fixed configurations for maneuvering at low speed such as that shown in Figure 6-10. All such configurations sacrifice control over one or more axes. Pitch control is sacrificed for the configuration shown in the figure; however, the vehicle's substantial metacentric restoring moment makes this configuration viable.

\subsection{Non-Linear Approaches}

Based on the previous section, we conclude that the partial failure of the classical linear controller proposed is a result of the vehicle's inherent input mapping nonlinearity. This non-linearity is a consequence of the combined foil/thruster actuator idea, and although it ultimately limits the effectiveness of classical control techniques, it is also enables a highly maneuverable and efficient vehicle. Furthermore, the analy- 


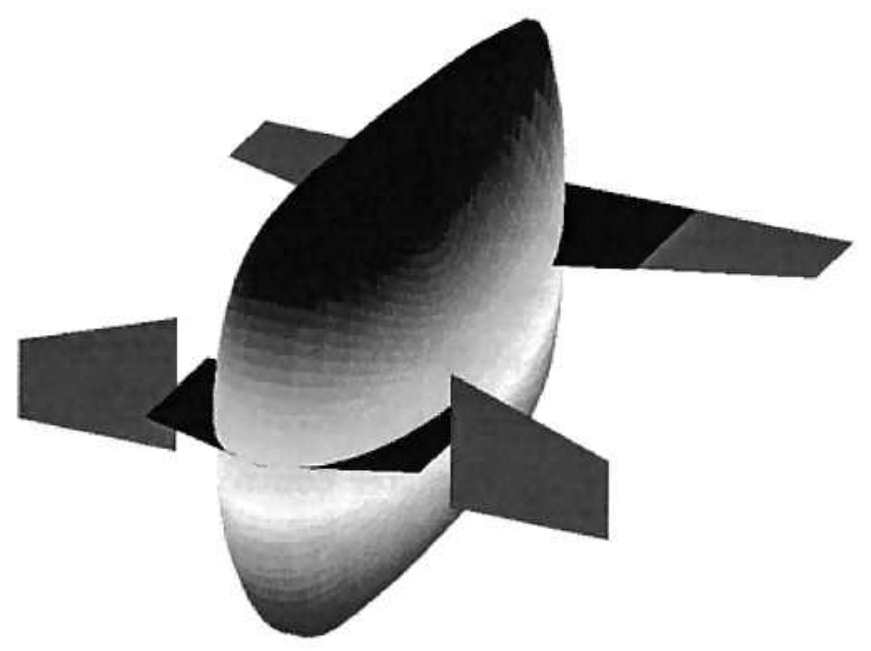

Figure 6-10: A Foils-Fixed Configuration Suitable for Low Speed Maneuvering.

sis in Section 5.3 suggests the non-linearity can be compensated for by appropriately modulating control over each vehicle axis between the foils and thrusters depending on gross foil angle. Intuitively, as foil angle becomes large, pitch damping and depth control should be assigned to the thrusters, and the thrusters should no longer attempt to control yaw.

To aid the development of such a controller, we define the following control variables:

$$
\begin{aligned}
& z_{c t r l}=k_{P_{z}}\left(z_{d}-z\right)-k_{D_{z}} w+k_{I_{z}} \int_{t}\left(z_{d}-z\right) d t \\
& \theta_{c t r l}=-k_{D_{\theta}} q \\
& \psi_{c t r l}=k_{P_{\psi}}\left(\psi_{d}-\psi\right)-k_{D_{\psi}} r
\end{aligned}
$$

which are simply the control laws developed previously, but without specified input mappings.

We have seen that the control law for $\phi_{a f_{z}}$, equation (6.15), commands the foils reasonably, even well outside the linear range it was designed for, if its output is 
clipped to $\pm 90^{\circ}$. We apply clipping, but otherwise leave this control law intact:

$$
\phi_{a f_{z}}=z_{c t r l}, \quad-90^{\circ} \leq \phi_{a f_{z}} \leq 90^{\circ} .
$$

The analysis in Section 5.3 shows that input mappings are trigonometrically modulated between vehicle axes. Thus it is natural to choose trigonometric functions for control modulation. The gross foil angle is regulated by $\phi_{a f_{z}}$ above, making it the natural choice for the modulating variable.

Beginning with foil pitch damping $\phi_{a f_{\theta}}$, we propose the modulated control law:

$$
\phi_{a f_{\theta}}=\cos \left(\phi_{a f_{z}}\right) \theta_{c t r l}
$$

The corresponding thrust-based pitch damper is then

$$
h_{\theta}=\sin \left(\phi_{a f_{z}}\right) \theta_{c t r l}
$$

where the individual thrusts satisfy

$$
h_{\theta}=h_{f p}+h_{f s}-\left(h_{a p}+h_{a s}\right) .
$$

Thrust control over depth can be implemented as

$$
h_{z}=\sin \left(\phi_{a f_{z}}\right) z_{c t r l}
$$

where the individual thrusts satisfy

$$
h_{z}=h_{f p}+h_{f_{s}}+h_{a p}+h_{a s} .
$$

Finally, differential thrust control over yaw is detrimental at high foil angles and must be reduced:

$$
h_{\psi}=\cos \left(\phi_{a f_{z}}\right) \psi_{c t r l} .
$$

The individual thrusts are as defined in equation (6.22).

The transformed input variables $\phi_{a f_{z}}$ and $\phi_{a f_{\theta}}$ map to the foil angles $\phi_{f f}$ and $\phi_{a f}$ as before (equation (6.7)). The individual thrust commands are composed of the summed contributions of each controller above, and the open loop nominal thrust 
given in equation (6.1). Explicitly, the thrust commands are given by:

$$
\boldsymbol{h}=\left[\begin{array}{l}
-a_{11} U_{o}^{2} \\
-a_{11} U_{o}^{2} \\
-a_{11} U_{o}^{2} \\
-a_{11} U_{o}^{2}
\end{array}\right]+\left[\begin{array}{c}
1 \\
1 \\
-1 \\
-1
\end{array}\right] h_{\theta}+\left[\begin{array}{l}
1 \\
1 \\
1 \\
1
\end{array}\right] h_{z}+\left[\begin{array}{c}
1 \\
-1 \\
1 \\
-1
\end{array}\right] h_{\psi}
$$

Initial results using this form of control show promise; however, no investigation of the optimality of the modulation functions proposed has been carried out, nor has a systematic exploration of system stability been attempted. 


\section{Chapter 7}

\section{Conclusions}

In this work, we presented the development and analysis of an analytic model of the Sentry AUV. Our primary methodology for analysis was through the design of a linear controller, whose behavior was investigated in simulation and as implemented on a $\frac{1}{4}$-scale physical model.

The Sentry AUV represents a radical departure from conventional AUV design, particularly with respect to actuation. The vehicle's combined foil/thruster actuators have the potential to produce a both maneuverable and efficient vehicle well suited to survey work over rough topography. Capitalizing on this; however, requires an understanding of the vehicles dynamics. Thus, our modeling goals were to develop a model sufficiently accurate in terms of the mission profile, and to identify critical vehicle behaviors influencing successful completion of its mission.

The analytical vehicle model was developed with structural accuracy in mind, and under the requirement that it handle a large range of vertical plane velocities. Our basic approach was to model the vehicle as having constant inertial and added mass characteristics, but varying lift and drag characteristics dependent upon control foil angle. To this end we presented a technique for the computation of quadratic lift and drag forces generated by a $2 \mathrm{D}$ section, and applied it to the derivation of a matrix of integral expressions for the quadratic lift and drag of the vehicle. This technique allowed us to account for differing fluid velocities across each section of the hull and foils in computing the resultant lift and drag forces. Where possible, we compensated for the true 3D nature of the flow around the vehicle with appropriate choices of leading coefficients. For hydrodynamic forces that could not be estimated in this way, we relied on empirical results.

The non-linear analytical model developed was linearized about near-horizontal flight conditions to enable a linear analysis of vehicle flight characteristics. For near- 
horizontal flight, the model was shown to decouple into three lightly interacting subsystems.

Based on analysis of the pitch-depth subsystem, the vehicle was shown to be more suited to direct depth change than indirect depth change via non-zero pitch. Moreover, the pitch response of the vehicle was shown to be minimized by the appropriate choice of the aft foil angle to forward foil angle ratio, but well damped in any case for smooth desired trajectories. As forward speed decreases, pitch became less damped.

Vehicle pitch response during a depth change remains non-ideal in one respect. For the control foil angle ratio selected, steady state pitch during a shallow climb opposes climb angle. The result is increased drag and reduced efficiency during a shallow climb. The basic reason for this behavior is the larger moment arm of the aft control foil compared to the forward foil. Fortunately, the large hydrostatic restoring moment of the vehicle limits the steady-state pitch to an acceptably small angle.

Analysis of the lateral subsystem indicated undesirable yaw instability, detrimental to control at high angle of attack. As noted in Section 4.3.1, our model overestimates the magnitude of the lateral destabilizing moment, rendering our analysis qualitative. An improved model of lateral hull hydrodynamics would allow a quantitative evaluation.

Based on these decoupled linearized models, classical linear controllers were designed and validated by simulation and implementation on the physical model. In simulation, the closed-loop system was shown to become more stiff and more damped with increasing speed due to the quadratic dependence of lift generated by the foils on speed. Closed loop simulations conducted at high angle of attack verified the vehicle's predicted maneuverability in the vertical plane. Ultimately we determined the vehicle's trigonometric input structure limited the achievable performance of a classical linear controller, but also suggested a route towards a more capable controller.

\section{$7.1 \quad$ Further Work}

The remainder of this chapter is devoted to recommendations for further modeling work and validation and to recommended directions for controller development.

\subsubsection{Model Verification}

Parameter errors are inevitable in the derived analytical model. One particular area of concern is the lateral hull hydrodynamics which are deficient for lack of an estimate of 
the expected stabilizing suction forces created along the aft portion of the hull. Once the full scale vehicle becomes available, efforts should be made to identify parametric inaccuracies in our model. The bifurcations identified while considering different modes of depth control could prove useful in adjusting model parameters.

Validating the structure of the full non-linear model is a more difficult task, necessitating the study of vehicle motions at high angles of attack.

A steady-state analysis of propeller and foil interaction was used to justify the assumption that the foils and thrusters could be treated independently in terms of their hydrodynamics. The validity of that analysis should be explored experimentally.

\subsubsection{Control Design}

Further control design must compensate for the non-linear mapping between inputs and vehicle axes. The trigonometric modulation scheme suggested in Section 6.4 should be pursued with particular attention paid to control over yaw at high angles of attack. Without additional actuation, at least one foil must be at $\phi_{f}<90^{\circ}$ to maintain a direct input into the yaw axis. Regardless, at larger control foil angles input coupling into roll becomes significant. Specifying a nominal control foil angle as opposed to relying on the serendipitous behavior of the linear controller in this respect may provide more intelligent control over foil angle during steep climbs. 


\section{Appendix A}

\section{Expressions for Arbitrary Control Foil Angles}

The following provides derivations of vehicle inertial, added mass, and gravitational quantities for arbitrary, but static control foil angles. Insofar as allowing these expressions to be dynamically dependent on control foil angle would violate conservation of energy, these expressions are provided for the study of nominally static vehicle configurations other than $\phi_{f}=0$.

\section{A.1 Rigid Body Inertia}

The inertial characteristics of the complete vehicle change to for changing control foil angles. The degree to which they change is dependent on the mass and inertial characteristics of the foils, in particular on any displacement between each foil axis and its center of gravity ${ }^{f} \boldsymbol{r}_{G_{f f, a f}}$ and on the inertia tensor associated with each foil ${ }^{f} I_{o_{f}}$.

The vector to the center of mass of a body is defined as

$$
r_{G} \equiv \frac{1}{m} \int_{V} \rho r d V
$$

where the integral is over the vehicle volume $V$ and vehicle density $\rho$ varies with $\boldsymbol{r}$. 
In terms of the body and control foils,

$$
\begin{aligned}
\boldsymbol{r}_{\boldsymbol{G}}\left(\boldsymbol{\phi}_{\boldsymbol{f}}\right) & =\frac{1}{m_{b}+2 m_{f}}\left[\int_{V_{b}} \rho_{b}{ }^{b} \boldsymbol{r}_{\boldsymbol{b}} d V+\int_{V_{f f, a f}} \rho_{f}{ }^{b} \boldsymbol{r}_{\boldsymbol{f}} d V\right] \\
& =\frac{1}{m_{b}+2 m_{f}}\left[m_{b}{ }^{b} \boldsymbol{r}_{\boldsymbol{G}_{b}}+\left.m_{f}\left({ }^{b} \boldsymbol{r}_{f_{G}}\right)\right|_{f f, a f}\right]
\end{aligned}
$$

where

$$
{ }^{b} r_{f_{G}}={ }^{b} r_{f_{0}}+R_{f}{ }^{f} r_{G_{f}} .
$$

In vector integral form, the inertia tensor of a body about the origin of a bodyfixed frame is

$$
\boldsymbol{I}_{\boldsymbol{o}}=-\int_{V}\left(\boldsymbol{r}^{T} \boldsymbol{r} I_{3 \times 3}-\boldsymbol{r} \boldsymbol{r}^{T}\right) \rho d V
$$

Furthermore, by the parallel axis theorem

$$
\boldsymbol{I}_{\boldsymbol{o}}=\boldsymbol{I}_{\boldsymbol{c}}-m\left(\boldsymbol{r}_{G} \boldsymbol{r}_{G}^{T}-\boldsymbol{r}_{G}^{T} \boldsymbol{r}_{G} I_{3 \times 3}\right)
$$

where $\boldsymbol{I}_{\boldsymbol{c}}$ denotes the moment of inertia of a body about its center of mass.

Using these expressions, the inertia tensor of each control foil can be expressed as

$$
\begin{aligned}
& { }^{f} \boldsymbol{I}_{\boldsymbol{c}_{f f, a f}}={ }^{f} \boldsymbol{I}_{\boldsymbol{o}_{\boldsymbol{f}}}+m_{f}\left({ }^{f} \boldsymbol{r}_{\boldsymbol{G}_{f}}{ }^{f} \boldsymbol{r}_{\boldsymbol{G}_{f}}{ }^{T}-{ }^{f} \boldsymbol{r}_{\boldsymbol{G}_{f}}{ }^{T f} \boldsymbol{r}_{\boldsymbol{G}_{f}} I_{3 \times 3}\right) \\
& { }^{b} \boldsymbol{I}_{\boldsymbol{o}_{f f, a f}}=\boldsymbol{R}_{\boldsymbol{f}} \boldsymbol{I}_{\boldsymbol{c}_{\boldsymbol{f}}} \boldsymbol{R}_{\boldsymbol{f}}-m_{f}\left({ }^{b} \boldsymbol{r}_{f_{G}}{ }^{b} \boldsymbol{r}_{f_{G}}{ }^{T}-{ }^{b} \boldsymbol{r}_{\boldsymbol{f}_{G}}{ }^{T b} \boldsymbol{r}_{f_{G}} I_{3 \times 3}\right)
\end{aligned}
$$

Thus for the complete vehicle,

$$
\boldsymbol{I}_{\boldsymbol{o}}\left(\phi_{f}\right)={ }^{b} \boldsymbol{I}_{o_{b}}+{ }^{b} \boldsymbol{I}_{o_{f f}}+{ }^{b} \boldsymbol{I}_{o_{a f}}
$$

The dependence on $\phi_{f}$ of the above is implicit in the expressions for $R_{f f}$ and $R_{a f}$ given in equation (3.6).

\section{A.2 Combined Added Mass}

The added mass matrix of the entire vehicle, composed of the body and two pairs of foils, can be derived by considering the kinetic energy of the surrounding fluid:

$$
T=\frac{1}{2} \nu^{T} \boldsymbol{M}_{\boldsymbol{A}}\left(\phi_{f}\right) \boldsymbol{\nu}
$$

As indicated, the added mass of the complete vehicle $M_{A}$ is dependent on foil angle. 
The foil velocity ${ }^{f} \boldsymbol{\nu}_{f}$ can be expressed in the body frame as:

$$
{ }^{b} \boldsymbol{\nu}_{f}=\left[\begin{array}{c}
{ }^{b} \boldsymbol{\nu}_{\boldsymbol{b}_{1}}+{ }^{b} \boldsymbol{\nu}_{\boldsymbol{b}_{2}} \times{ }^{b} \boldsymbol{r}_{f_{0}} \\
{ }^{b} \boldsymbol{\nu}_{b_{2}}
\end{array}\right]
$$

Furthermore,

$$
{ }^{b} \nu_{f}=\left[\begin{array}{cc}
R_{f} & 0_{3 \times 3} \\
0_{3 \times 3} & R_{f}
\end{array}\right]{ }^{f} \nu_{f}
$$

Combining (A.11) with (A.10), the kinetic energy of the surrounding fluid can be written

$$
T=\frac{1}{2}\left[\left[{ }^{b} \boldsymbol{\nu}_{\boldsymbol{b}}^{T} \boldsymbol{M}_{\boldsymbol{A}_{\boldsymbol{b}}}{ }^{b} \boldsymbol{\nu}_{\boldsymbol{b}}+{ }^{b} \boldsymbol{\nu}_{\boldsymbol{b}}^{T} \boldsymbol{U}^{T} \boldsymbol{M}_{\boldsymbol{A}_{\boldsymbol{f}}} \boldsymbol{U}^{b} \boldsymbol{\nu}_{\boldsymbol{b}}\right]\right.
$$

where

$$
\boldsymbol{U}\left(\boldsymbol{\phi}_{\boldsymbol{f}}\right)=\left[\begin{array}{cc}
\boldsymbol{R}_{\boldsymbol{f}} & 0_{3 \times 3} \\
0_{3 \times 3} & \boldsymbol{R}_{\boldsymbol{f}}
\end{array}\right]^{-1}\left[\begin{array}{cc}
I_{3 \times 3} & -\boldsymbol{S}\left({ }^{\boldsymbol{b}} \boldsymbol{r}_{f_{0}}\right) \\
0_{3 \times 3} & I_{3 \times 3}
\end{array}\right]
$$

From (A.12), the added mass matrix for the complete vehicle is then

$$
M_{A}=M_{A_{b}}+U^{T} M_{A_{f}} U
$$

\section{A.3 Gravitational Restoring Moment}

As mentioned in the text (Section 4.5), the gravitational restoring moment is invariant with control foil angle for foil centers of mass and centers of buoyancy located on axis. This condition is desirable to avoid steady state non-zero trim and to reduce the required control torques.

Should this condition not be met in the final design, the vector of gravitational restoring torques becomes

$$
\boldsymbol{g}(\boldsymbol{\eta})=\left[\begin{array}{c}
0 \\
0 \\
0 \\
\left(z_{G}-z_{B}\right) W \cos \theta \sin \phi \\
\left(z_{G}-z_{B}\right) W \sin \theta+\left(x_{G}-x_{B}\right) W \cos \theta \cos \phi \\
-\left(x_{G}-x_{B}\right) W \cos \theta \sin \phi
\end{array}\right]
$$

where $x_{G}, x_{B}, z_{G}$, and $z_{B}$ are given by the components of $\boldsymbol{r}_{\boldsymbol{G}}\left(\boldsymbol{\phi}_{\boldsymbol{f}}\right)$ and $\boldsymbol{r}_{\boldsymbol{B}}\left(\boldsymbol{\phi}_{\boldsymbol{f}}\right)$. The 
expression for $\boldsymbol{r}_{\boldsymbol{G}}$ is derived above in Section A.1. A parallel derivation yields

$$
\boldsymbol{r}_{\boldsymbol{B}}\left(\phi_{f}\right)=\frac{1}{B_{b}+2 B_{f}}\left[B_{b}{ }^{b} \boldsymbol{r}_{B_{b}}+\left.B_{f}\left({ }^{b} \boldsymbol{r}_{f_{0}}+\boldsymbol{R}_{f}{ }^{f} \boldsymbol{r}_{B_{f}}\right)\right|_{f f, a f}\right] .
$$




\section{Appendix B}

\section{Physical Model}

This appendix describes the $\frac{1}{4}$-scale physical model of the Sentry vehicle used to investigate the vehicle design and validate controller concepts.

\section{B.1 Similitude}

Geometric similitude between the physical model and the full-scale vehicle is satisfied except for the tether, thruster nacelles, and exposed cabling of the physical model. The tether constitutes the single most important difference between the dynamics of the physical model and the full-scale vehicle.

The hydrodynamic behavior of the physical model relative to the full-scale vehicle depends on both the Reynolds number $R n_{l}=\frac{\rho U l}{\mu}$ and Froude number $F r_{l}=\frac{U}{\sqrt{g l}}$ " The Reynolds number determines the nature of the fluid flow around the vehicle (laminar or turbulent) and influences lift and drag forces, while the Froude number is important because of the large hydrostatic restoring moment of the vehicle. The Reynolds number scales linearly with velocity, implying the physical model would have to move at $4 \mathrm{~m} / \mathrm{sec}$ to match the Reynolds number of the full-scale vehicle cruising at $1 \mathrm{~m} / \mathrm{s}$. As the physical model is capable of at most $0.75 \mathrm{~m} / \mathrm{sec}$, viscous fluid forces will influence the fluid forces acting on the physical model to a greater extent than for the full-scale vehicle. The influence of the hydrostatic restoring moment is dependent on $\mathrm{CB} / \mathrm{CG}$ separation. In terms of Froude scaling, this implies a required CG/CB separation of

$$
z_{G B_{\text {model }}}=z_{G B_{\text {veh }}} \sqrt{\frac{U_{\text {model }}}{U_{\text {veh }}}}
$$

for the physical model. For a physical model speed of $0.5 \mathrm{~m} / \mathrm{s}$ and full-scale cruising speed of $1 \mathrm{~m} / \mathrm{s}$, the required CB/CG separation for Froude scaling would place the 
$\mathrm{CG}$ of the model at $0.14 \mathrm{~m}$ from the midplane. The actual $\mathrm{CB} / \mathrm{CG}$ separation of the model could not be increased beyond $5.5 \mathrm{~m}$.

Given these discrepancies between the physical model and the full-scale vehicle, results from the model must be regarded as qualitative.

\section{B.2 Design}

The original version of the physical model was built by Serfass [19]; however it lacked any on-board sensors and the thrusters and foils were controlled by an operator using simple on/off switches. To facilitate closed-loop operation, the original foil actuators were replaced with waterproofed proportional hobby-grade servos, four hobby-grade speed controls were added for proportional thruster control, and on-board depth/attitude sensors were added along with the necessary electronics to interface serially to a PC. The original model was also retrofitted with a redesigned tether and thruster housings.

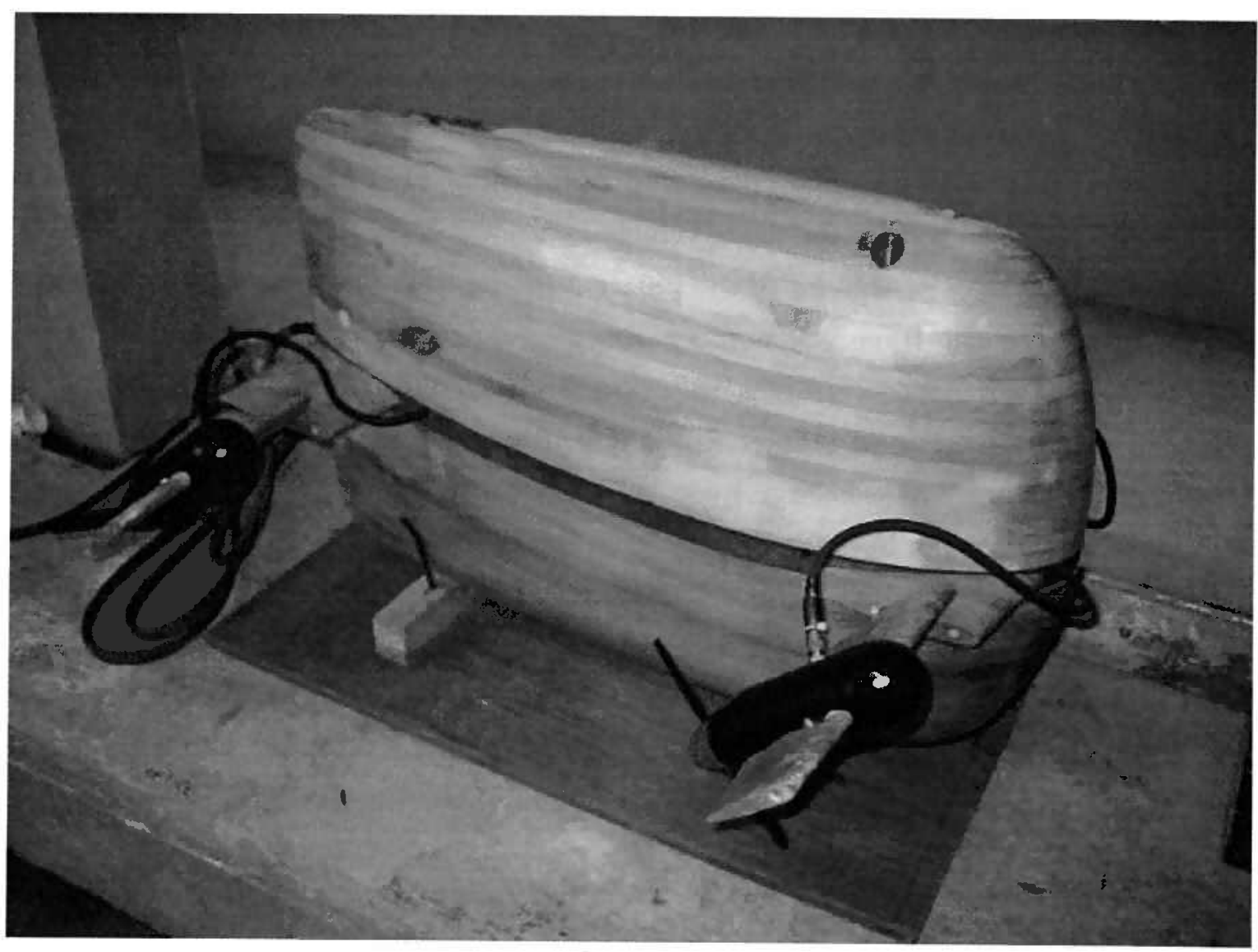

Figure B-1: $\frac{1}{4}$-Scale Physical Model of Sentry 
The hull and foils are laminated pine sealed with epoxy. The lower hull has space for a $15 \mathrm{lb}$. lead weight along with holes forward and aft for the lead shot used to trim the model. All other model components are mounted on the removable midplane shown in Figure B-2.

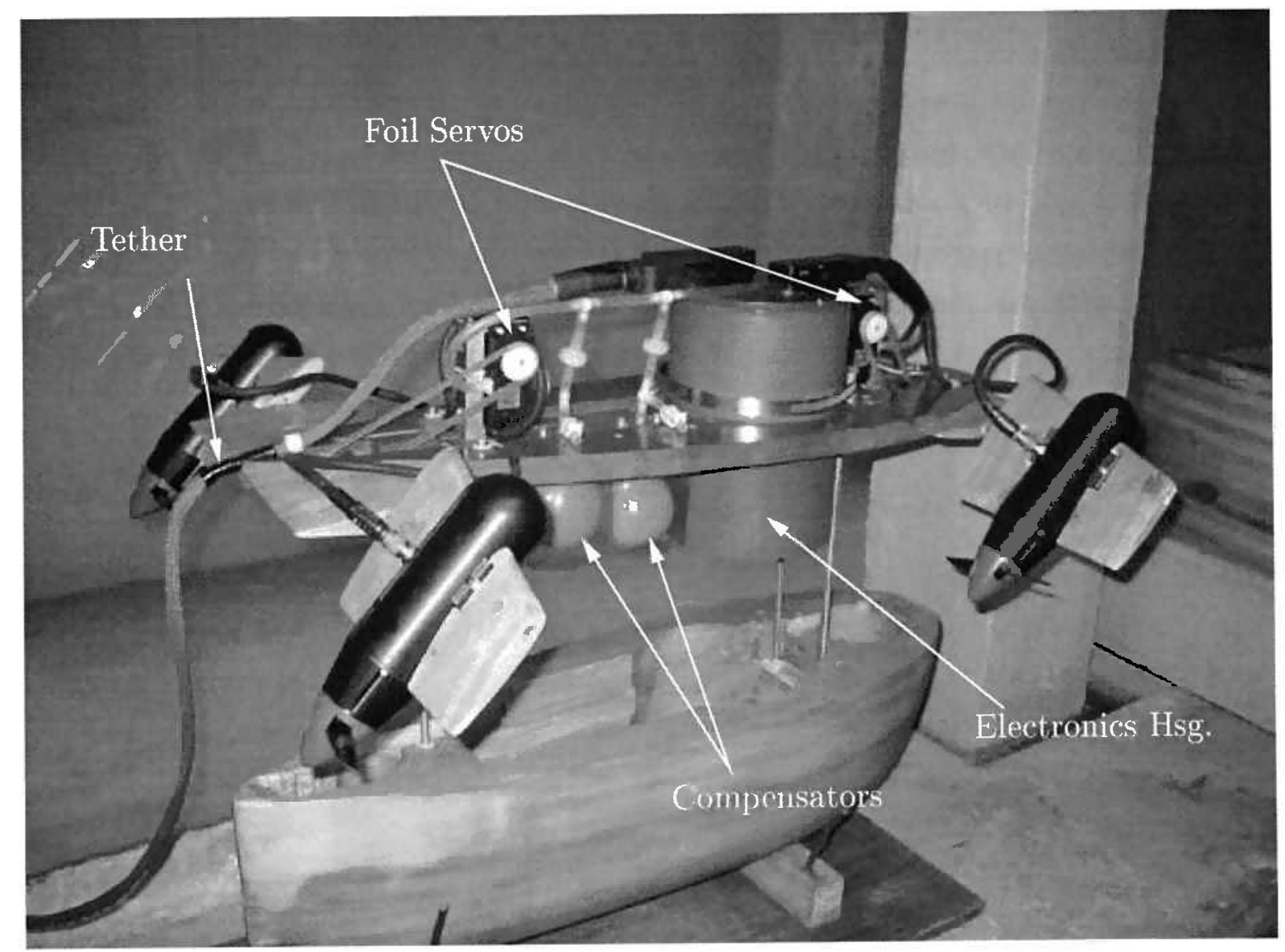

Figure B-2: Physical Model: Midplane

The foil actuators are Tower Hobbies TS-80 hobby-grade servos with internal closed-loop position control and maximum output torques of 343 oz-in at $6 \mathrm{~V}$. These are connected to the control foil shafts by toothed belts. The thruster speed controllers are 15 Vmaximum hobby-grade Tekin Rebel PWM reversible electronic speed controls. Standard R/C hobby control signals for the servos and speed controls are provided by an 8-bit serial interface board available from Scott Edwards Electronics, Inc.

The foil servos and thruster speed controls are stand-alone devices and as such provide no direct feedback. The foil servos were calibrated such that 8-bit commands covered their angular position range $\left(>270^{\circ}\right)$. Except at the limits of their range, 
the servos exhibited a linear relation between input command and output angle. No attempt was made to characterize the speed controls.

The foil servos were sealed by coating all seams with epoxy after replacing the stock shaft seal with an appropriately sized o-ring. To prevent leakage under pressure, the internal cavities of the servos are connected by tubing to two redundant air filled compensators.

The electronics housing (Figure B-3) contains four speed controls, serial interface board, depth sensor, and a Cross Bow Instruments 3-axis rate gyro, 3-axis accelerometer, and 2-axis inclinometer. The depth sensor is comprised of an DIP packaged SenSym absolute reference analog pressure sensor vented to the exterior of the housing, along with the necessary amplification circuitry and an A/D converter from DGH Corporation. The vehicle communicates with an exterior PC via four separate serial

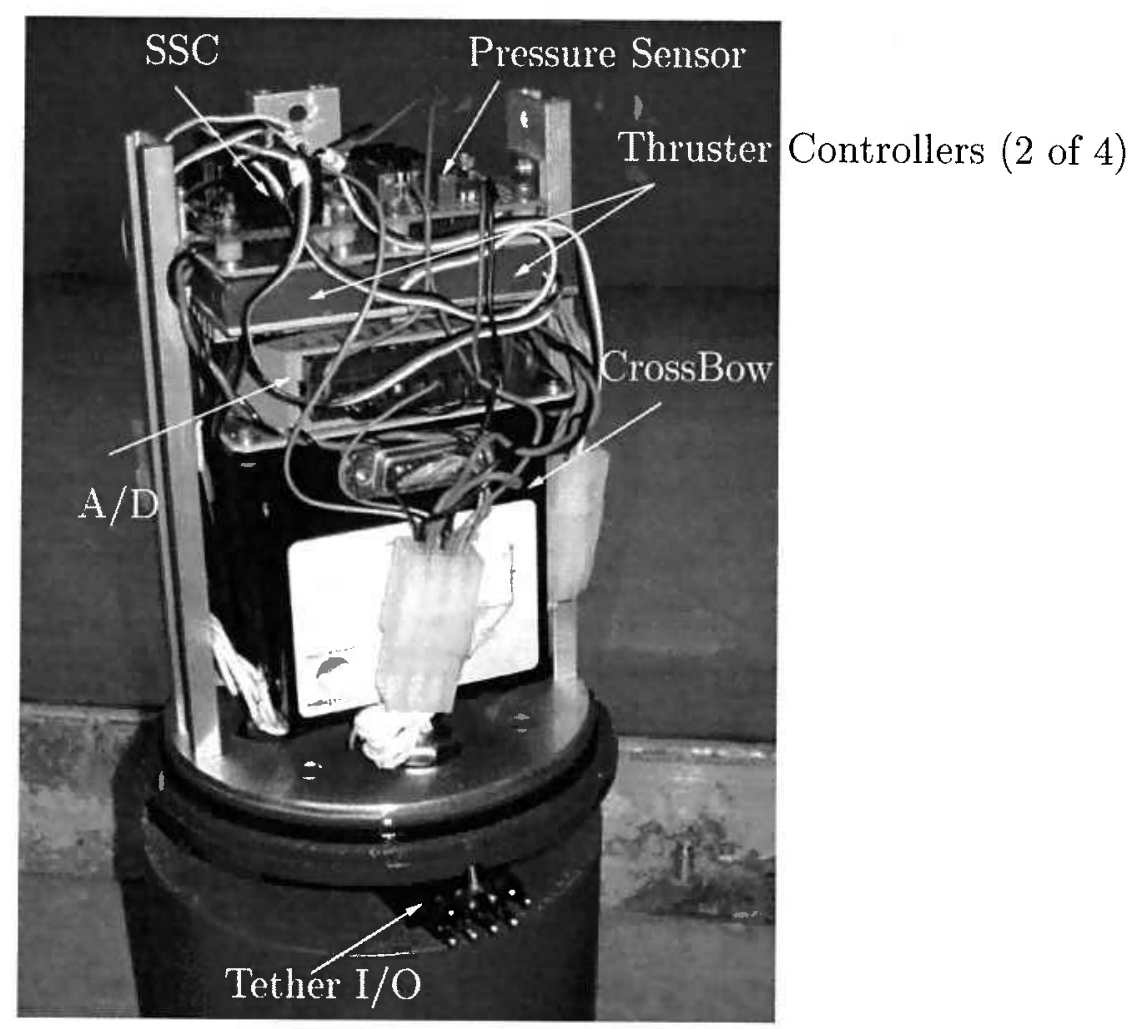

Figure B-3: Physical Model: Electronics Housing

lines in the tether.

The PC model interface enables joystick control of all vehicle actuators, closed loop depth control, and will also run command records directly from file. Input from an eight-axis joystick is mixed according to the values specified in the fields shown in the center panel of Figure B-4. This enables arbitrary mapping of the joystick axes to the 


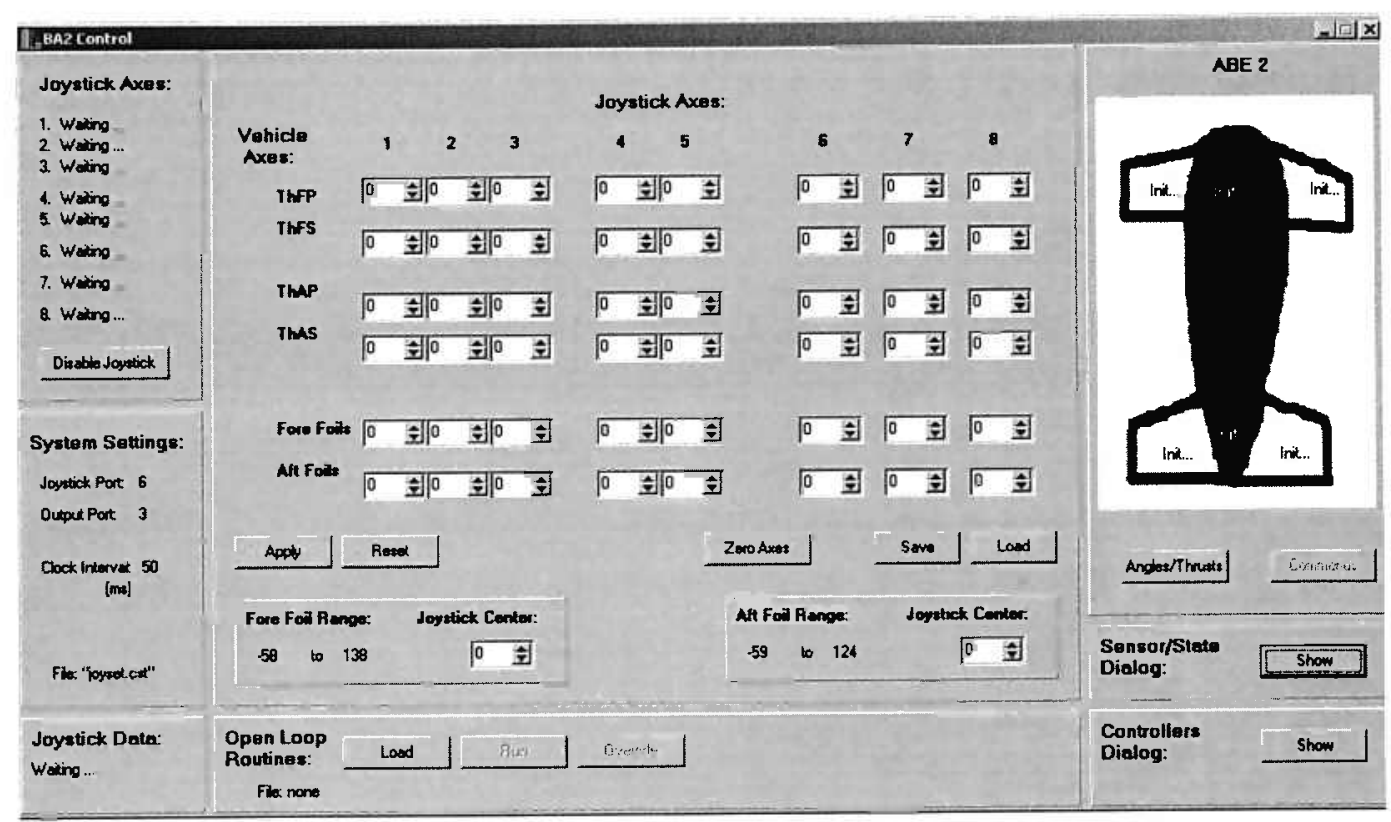

Figure B-4: Physical Model: Interface

model's actuators. Commands from any other sources (closed-loop controllers; openloop command records) are summed with joystick commands, clipped if necessary, and sent to the vehicle. Output commands are displayed on the graphic at the right of Figure B-4. All commands and sensor data are logged. 


\section{Appendix C}

\section{Simulation Code}

The following is MATLAB code used to calculate vehicle state derivatives from current state and inputs including a vector of disturbance forces. The equations of motion are implemented in matrix form. Integration of the $2 \mathrm{D}$ hydrodynamic forces and moments approximated using strip-theory is accomplished employing the vector expressions given in equations (4.41), (4.48) and (4.49). This code forms the core of a vehicle simulator implemented in MATLAB's Simulink environment.

Vehicle parameters are passed in a structure $\mathrm{S}$ containing the output of a separate vehicle parameters file sentry $m$ that computes the required quantities based on vehicle geometry and mass characteristics. In addition to regular coefficients, this file generates the vectors of coefficients and geometric parameters required to compute $2 \mathrm{D}$ sectional hydrodynamic forces. In the interests of space, we have not included code from sentry $m$.

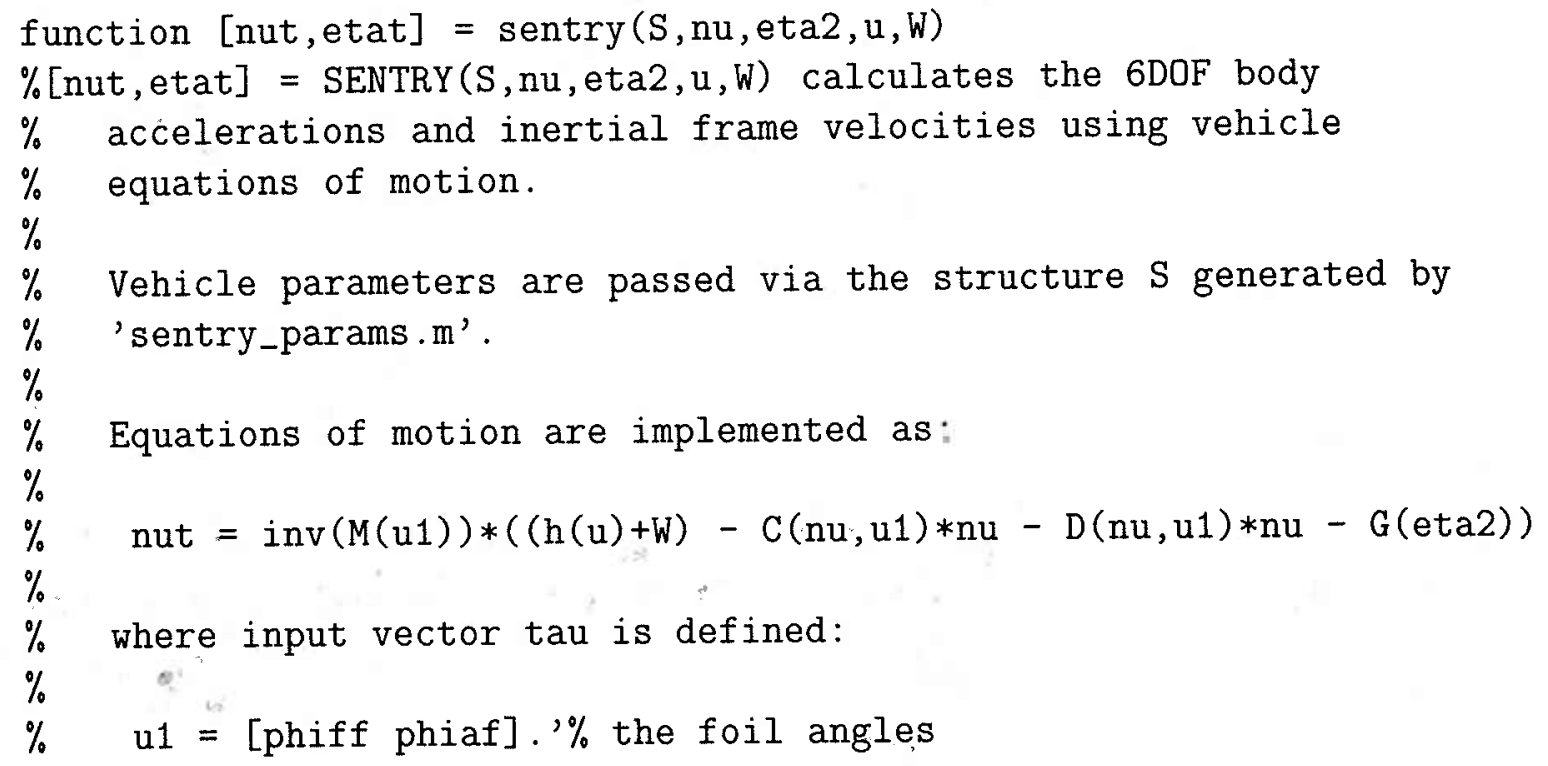




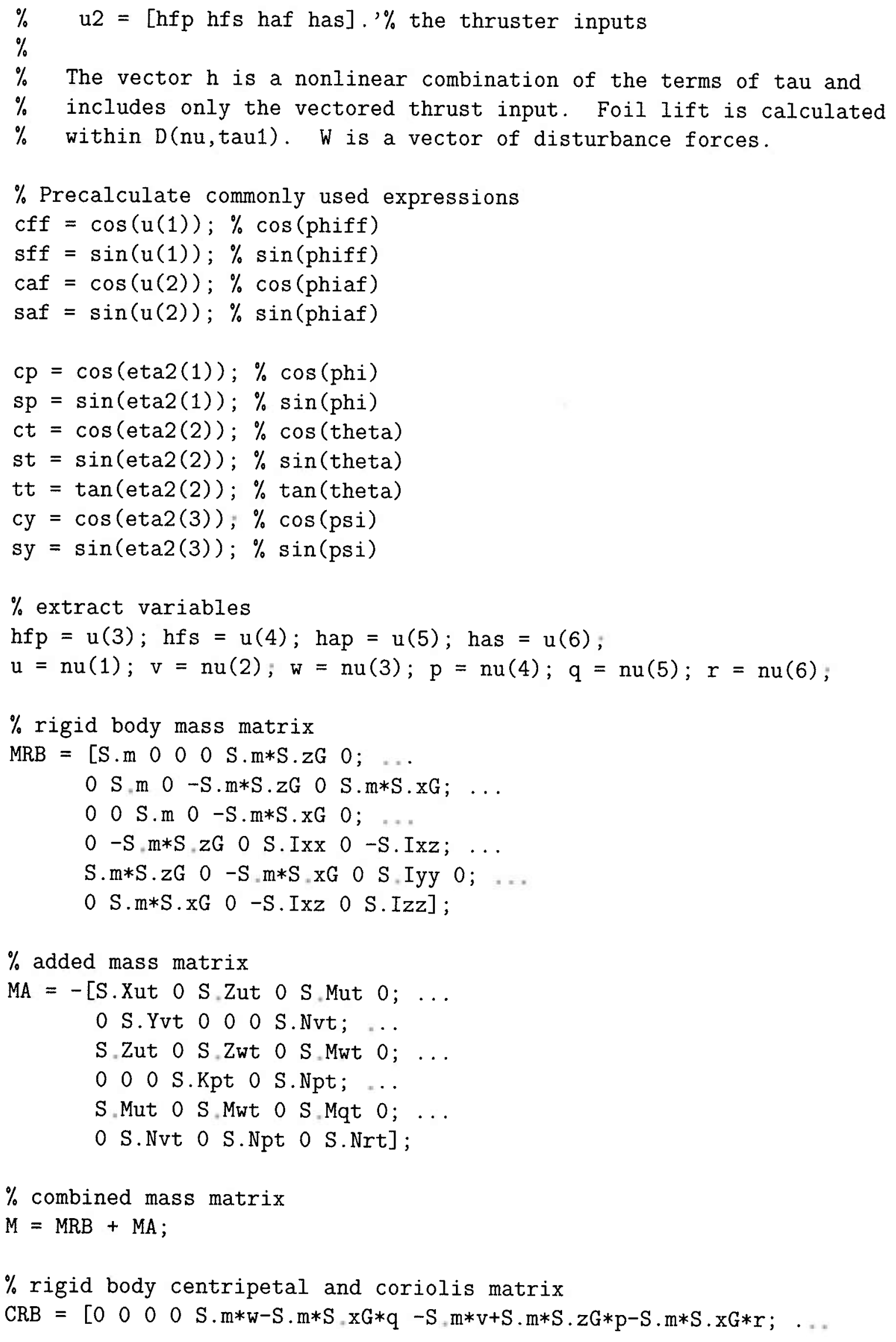




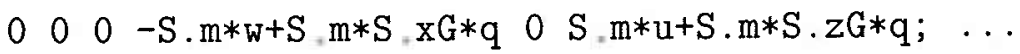

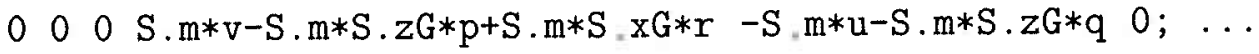

0 S. $m * w-S \cdot m * S, x G * q-S \cdot m * v+S \cdot m * S \cdot z G * p-S \cdot m * S \cdot x G * r ; \ldots$

$0-\mathrm{S} . \mathrm{m} * \mathrm{~S} . \mathrm{xG} * \mathrm{v}-\mathrm{S} . \mathrm{Ixz} * \mathrm{p}+\mathrm{S}$. Izz*r $\quad \mathrm{S} . \mathrm{m} * \mathrm{~S} . z \mathrm{zG} * \mathrm{u}-\mathrm{S} . \mathrm{m} * \mathrm{~S} . \mathrm{xG} * \mathrm{w}-\mathrm{S} . \mathrm{Iyy} * \mathrm{q} ; \ldots$

$-\mathrm{S} \cdot \mathrm{m} * \mathrm{w}+\mathrm{S} \cdot \mathrm{m} * \mathrm{~S} \cdot \mathrm{xG} * \mathrm{q}$ ○ $\mathrm{S} \cdot \mathrm{m} * \mathrm{u}+\mathrm{S} \cdot \mathrm{m} * \mathrm{~S} \cdot \mathrm{zG} * \mathrm{q} ; \ldots$

$\mathrm{S} . \mathrm{m} * \mathrm{~S} . \mathrm{xG} * \mathrm{v}+\mathrm{S} . \mathrm{Ixz} * \mathrm{p}-\mathrm{S} . \operatorname{Izz} * r \quad 0 \quad \mathrm{~S} . \mathrm{m} * \mathrm{~S} . z \mathrm{G} * \mathrm{v}+\mathrm{S} . \operatorname{Ixx} * \mathrm{p}-\mathrm{S} . \operatorname{Ixz} z r ; \ldots$

$\mathrm{S} . \mathrm{m} * \mathrm{v}-\mathrm{S} . \mathrm{m} * \mathrm{~S} . \mathrm{zG} * \mathrm{p}+\mathrm{S}, \mathrm{m} * \mathrm{~S}, \mathrm{xG} * \mathrm{r}-\mathrm{S} . \mathrm{m} * \mathrm{u}-\mathrm{S} . \mathrm{m} * \mathrm{~S} . z \mathrm{G} * \mathrm{q} 0 ; \quad \ldots$

$-\mathrm{S} . \mathrm{m} * \mathrm{~S}, z \mathrm{G} * \mathrm{u}+\mathrm{S} \cdot \mathrm{m} * \mathrm{~S} \cdot \mathrm{xG} * \mathrm{w}+\mathrm{S}$. Iyy*q $-\mathrm{S} \cdot \mathrm{m} * \mathrm{~S}, z \mathrm{G} * \mathrm{v}-\mathrm{S} \cdot \operatorname{Ixx} * \mathrm{p}+\mathrm{S} . \operatorname{Ixz} * \mathrm{r} \quad 0]$;

$\%$ added mass centripetal and coriolis matrix

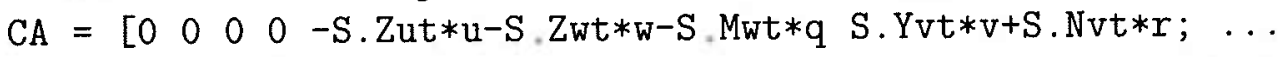

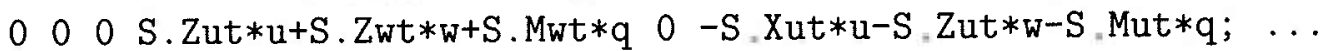

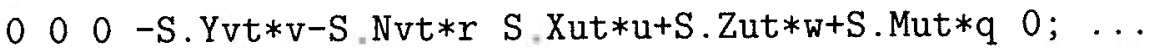

$0-S . Z u t * u-S, Z w t * w-S . M w t * q$ S. Yvt*v+S.Nvt*r; .

$0-S . M w t * v-S . N p t * p-S . N r t * r \quad S . N v t * w+S . M q t * q ; \quad \ldots$

S.Zut*u+S.Zwt*w+S.Mwt*q $0-S . X u t * u-S . Z u t * w-S . M u t * q$

S.Mwt*v+S.Npt*p+S.Nrt*r $0-S . M u t * v-S . K p t * p-S . N p t * r ; \quad \ldots$

$-S . Y v t * v-S . N v t * r \quad S . X u t * u+S . Z u t * w+S . M u t * q \quad 0 ; \quad \ldots$

$-S . N v t * w-S . M q t * q \quad S . M u t * v+S . K p t * p+S . N p t * r \quad 0]$;

$\%$ combined added mass and coriolis matrix

$\mathrm{C}=\mathrm{CRB}+\mathrm{CA}$;

$\%$ Generate $D(n u, u 1)$

$\%$ forward foil

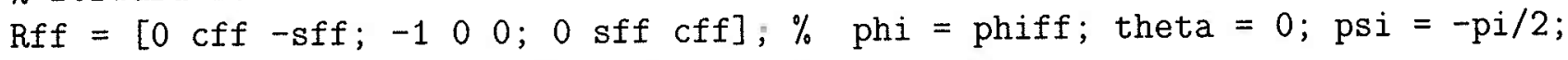

Dff $=$ LSQD360(S.rho, nu, S.rffo,Rff,S.xf,S.res, S.Llefx, ...

S.Ltefx, S.KLff, S.KDff, S.KDoff, S.CPf);

$\%$ aft foil

Raf $=[0$ caf -saf $;-100 ; 0$ saf caf $] ; \%$ phi = phiaf; theta $=0 ;$ psi $=-p i / 2$;

Daf $=$ LSQD360 (S.rho, nu, S.rafo, Raf, S xf, S res, S.Llefx, . .

S.Ltefx, S.KLaf, S.KDaf, S.KDoaf, S.CPf);

$\%$ forward root

$\operatorname{Rr}=\left[\begin{array}{lllllllll}0 & 1 & 0 ; & -1 & 0 & 0 ; & 0 & 0 & 1\end{array}\right] ; \%$ phi $=0 ;$ theta $=0 ; \operatorname{psi}=-\mathrm{pi} / 2 ;$

Dfr $=$ LSQD360(S.rho, nu, S.rffo,Rr, S.xfr, S.res, S.Llefrx, ...

S.Ltefrx, S.KLfr, S.KDfr, S.KDofr, S.CPr);

$\%$ aft root

Dar $=$ LSQD360 (S.rho, nu, S.rafo, Rr, S.xar, S.res, S.Llearx, . .

S.Ltearx, S.KLar, S.KDar, S. KDoar, S. CPr);

$\% \mathrm{xz}$ planar body lift and drag

Rbxy $=\left[\begin{array}{lllllllll}0 & 1 & 0 ; & 0 & 0 & 1 ; & 1 & 0 & 0\end{array}\right] ; \quad \%$ phi $=-p i / 2 ;$ theta $=-p i / 2 ;$ psi $=0 ;$

Dbxy $=$ LSQDIin(S.rho, nu, 0, Rbxy, S.zb, S.res, S.Lbnz, ...

S. Lbtz, S.KLbxy, S.KDobxy, S.CPbxy);

$\%$ yz planar body lift and drag

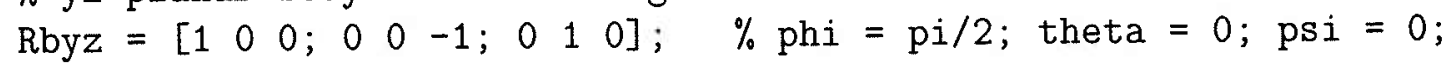




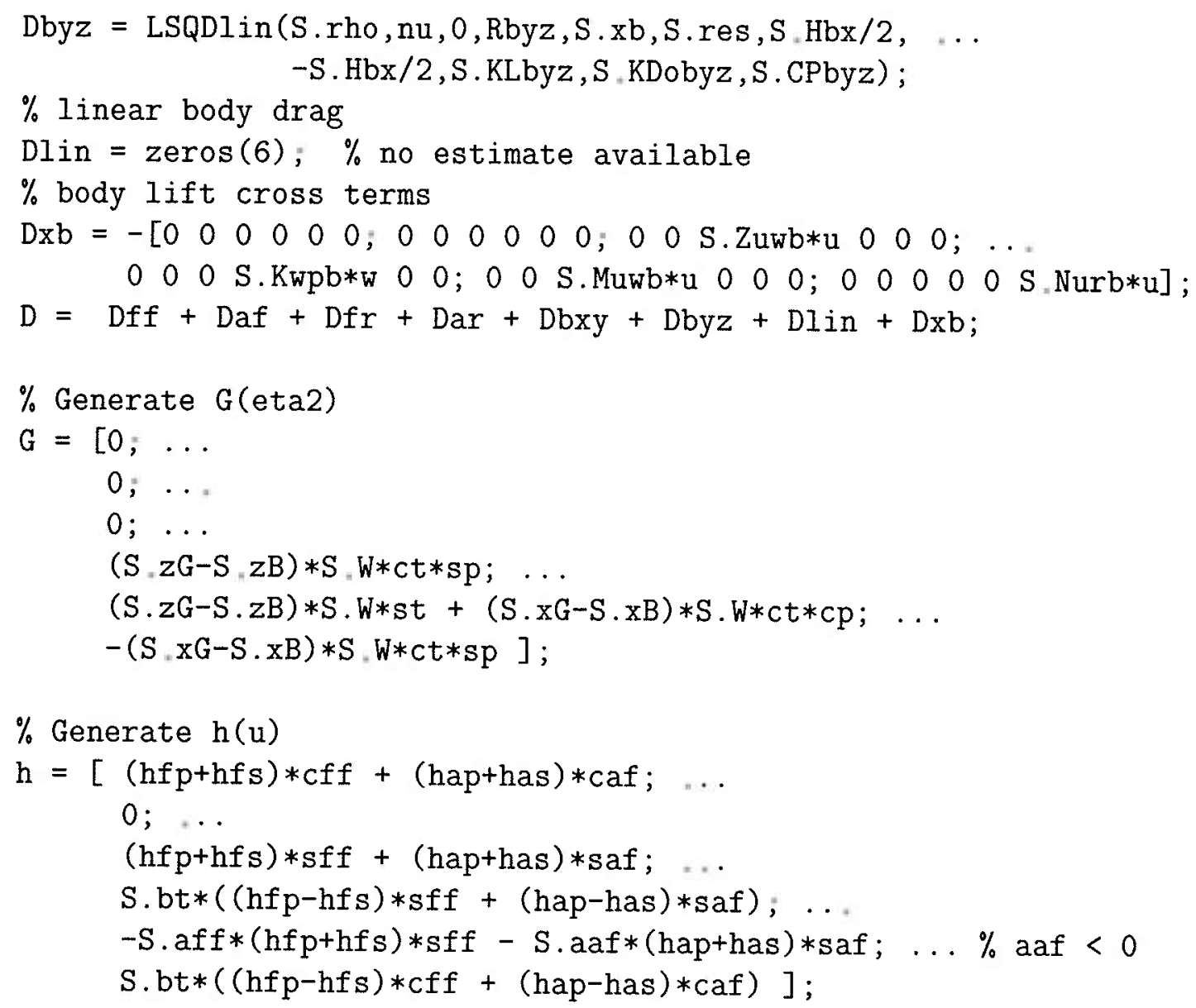




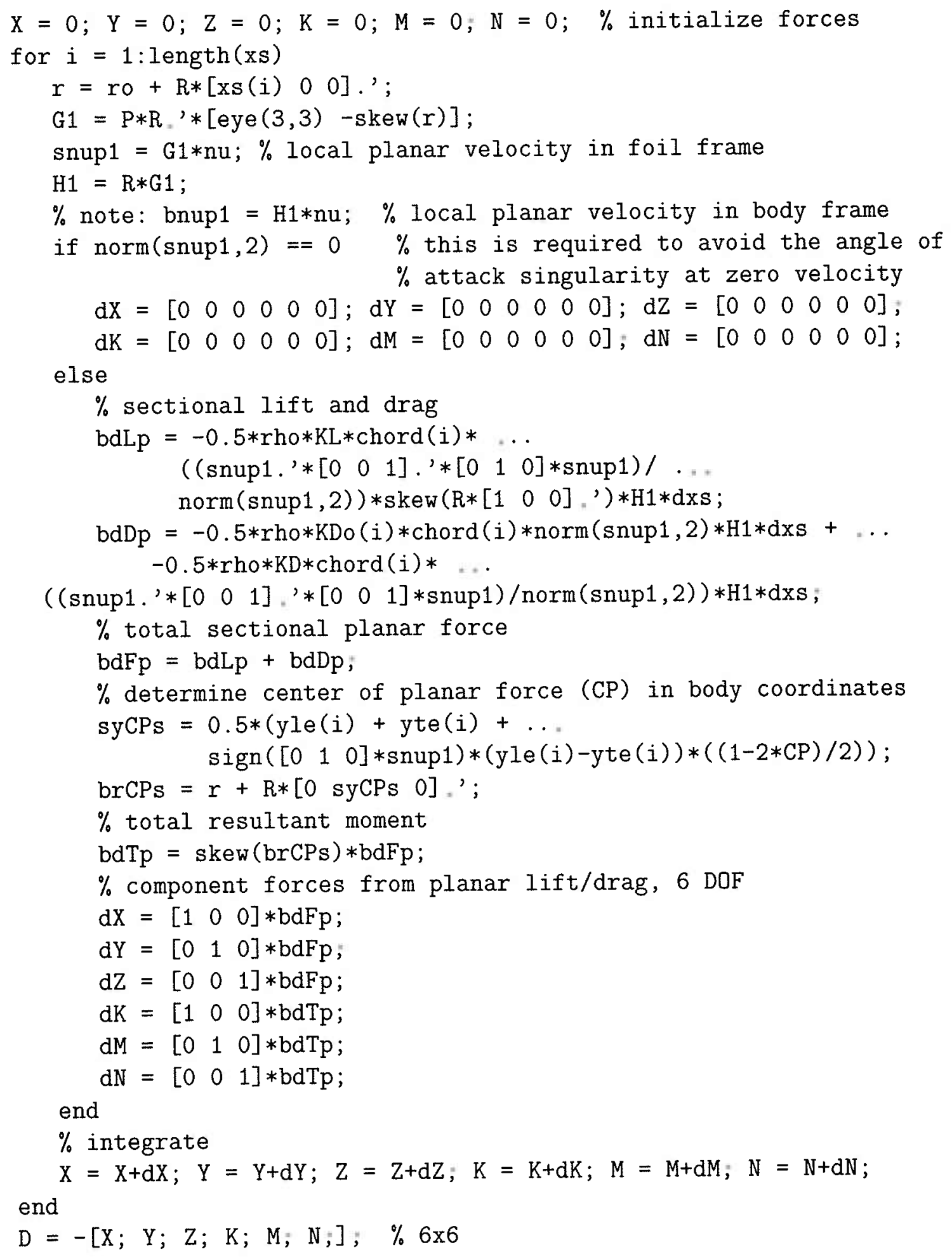




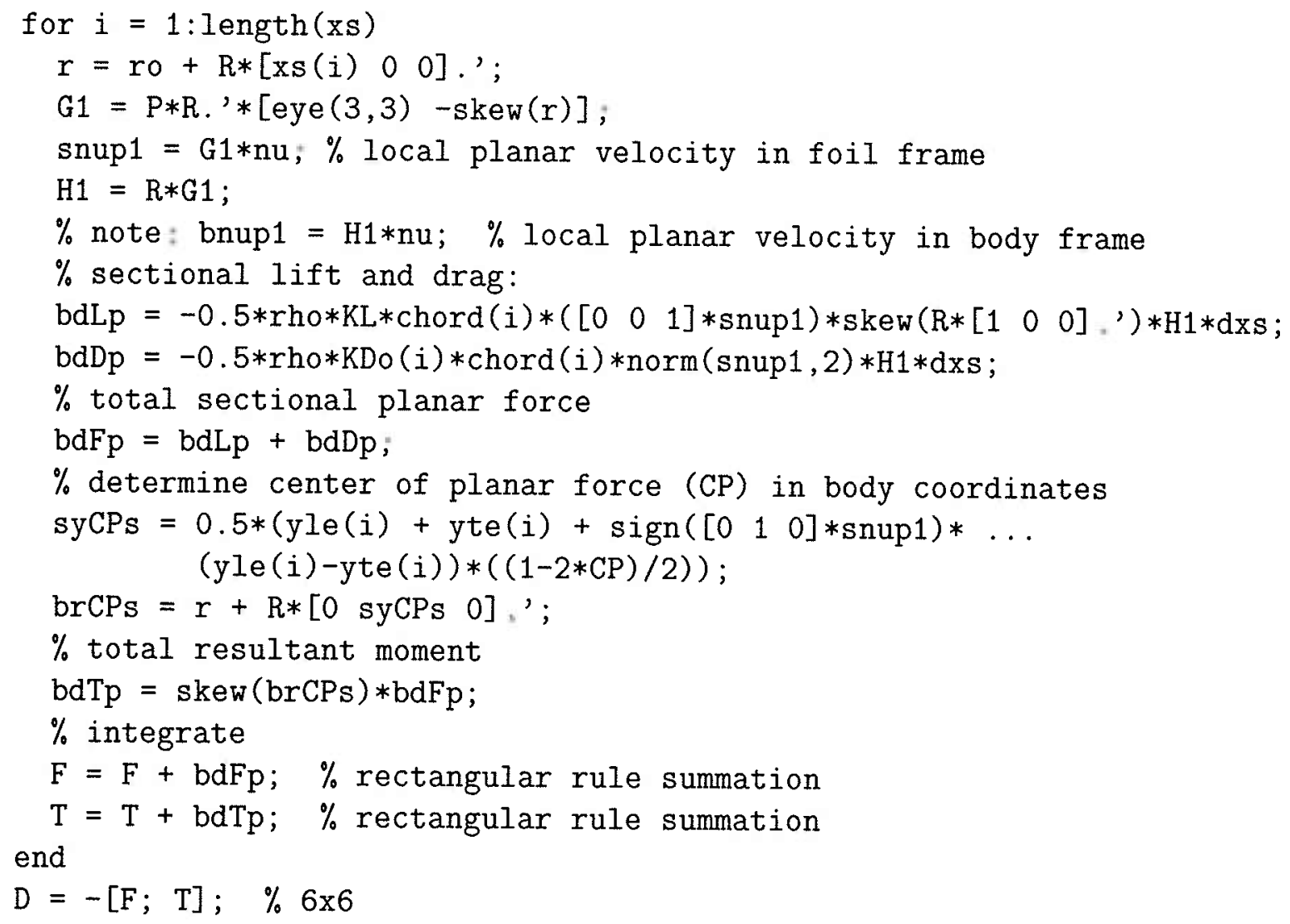




\section{Bibliography}

[1] M. A. Abkowitz. Stability and Motion Control of Ocean Vehicles. MIT Press, Cambridge, MA, 1972.

[2] S. Dubowsky and E. Papadopoulos. The Kinematics, Dynamics, and Control of Free-Flying and Free-Floating Space Robotic Systems. IEEE Transactions on Robotics and Automation, 9(5):531-543, Oct 1993.

[3] J. A. Fay. Introduction to Fluid Mechanics. MIT Press, Cambridge, MA, 1994.

[4] A. Filippone. Wings for All Speeds: Low Aspect Ratio Wings. March 1, 2002. <http://www . aerodyn . org/wings/larw . htm>.

[5] T. I. Fossen. Guidance and Control of Ocean Vehicles. John Wiley \& Sons, New York, 1994.

[6] K. R. Goheen. Modeling Methods for Underwater Robotic Vehicle Dynamics. Journal of Robotic Systems, 8(3):295-317, 1991.

[7] A. J. Healey and D. Lienard. Multivariable Sliding Mode Control for Autonomous Diving and Steering of Unmanned Underwater Vehicles. IEEE Journal of Oceanic Engineering, 18(3):327-338, Jul 1993.

[8] S. F. Hoerner. Fluid Dynamic Drag. Published by the author, 1965.

[9] S. F. Hoerner. Fluid Dynamic Lift. Published by the author, 1965.

[10] D. E. Humphreys and K. W. Watkinson. Methods of Estimating Vehicle Transfer Functions Requiring Only Geometric and Inertia Characteristics. In Proceedings IEEE Oceans 72 Conference, pages 220-223, Sep 1972.

[11] E. V. Lewis, editor. Principles of Naval Architecture. Society of Naval Architects and Marine Engineers, Jersey City, New Jersey, 1989.

[12] T. W. McLain and S. M. Rock. Experiments in the Hydrodynamic Modeling of an Underwater Manipulator. In Proceedings AUV '96, Monterey, CA, 1996.

[13] M. Nahon. A Simplified Dynamics Model for Autonomous Underwater Vehicles. In Proceedings 1996 Symposium on Autonomous Underwater Vehicle Technology, pages 373-379, Jun 1996. 
[14] J. N. Newman. Marine Hydrodynamics. MIT Press, 1977.

[15] N. S. Nise. Control Systems Engineering. Addison-Wesley, second edition, 1995.

[16] F. A. Papoulias and H. Papadimitriou. Nonlinear Studies of Dynamic Stability of Submarines in the Dive Plane. Journal of Ship Research, 39(4):347-356, December 1995.

[17] T. Prestero. Verification of a Six-Degree of Freedom Simulation Model for the REMUS Autonomous Underwater Vehicle. Master's thesis, MIT/WHOI Joint Program in Applied Ocean Science and Engineering, 2001. <http://web.mit. edu/ ${ }^{\sim}$ tprester/main pdf $>$.

[18] I. Schjølberg and T. I. Fossen. Modelling and Control of Underwater VehicleManipulator Systems. In Proceedings of the 3rd Conference on Marine Craft Maneuvering and Control (MCMC94), Southhampton, UK, Sep 1994.

[19] P. R. Serfass. An Investigation of the Design for the Autonomous Underwater Vehicle ABE II: Using a 1:4 Scale Controllable Prototype. Summer Student Fellowship final report, Advanced Engineering Laboratory, Dept. of Applied Ocean Phyisics and Engineering, Woods Hole Oceanographic Institution, Summer 2000.

[20] J.-J. E. Slotine and W. Li. Applied Nonlinear Control. Printice Hall, 1991.

[21] M. S. Triantafyllou and F. S. Hover. Maneuvering and Control of Surface and Underwater Vehicles, Sep 2000. MIT Course 13.42 Class Notes.

[22] K. W. Watkinson, N. S. Smith, and D. E. Humphreys. Thruster and Fin Control Authority Requirements. In Ninth International Symposium on Unmanned Untethered Submersible Technology (UUST95), Durham, NH, September 25-27 1995.

[23] D. Yoerger, A. Bradley, M.-H. Cormier, W. Ryan, and W. B. Fine-Scale Seafloor Survey in Rugged Deep-Ocean Terrain with an Autonomous Robot. In IEEE $\operatorname{ICRA}, 2000$.

[24] D. Yoerger, A. Bradley, M.-H. Cormier, W. Ryan, and B. Walden. High Resolution Mapping of a Fast Spreading Mid-Ocean Ridge with the Autonomous Benthic Explorer. In Proceedings of the 11th International Symposium on Unmanned Untethered Submersible Technology (UUST99), Durham, NH, 1999.

[25] D. R. Yoerger. Personal Communication, July 2002.

[26] D. R. Yoerger, A. M. Bradley, and B. B. Walden. The Autonomous Benthic Explorer (ABE): An AUV Optimized for Deep Seafloor Studies. In Proceedings of the Seventh International Symposium on Unmanned Untethered Submersible Technology (UUST91), pages 60-70, Durham, NH, 1991. 
[27] D. R. Yoerger, A. M. Bradley, B. B. Walden, H. Singh, and R. Bachmayer. Surveying a Subsea Lava Flow Using the Autonomous Benthic Explorer (ABE). International Journal of Systems Science, 29(10):1031-1044, 1998.

[28] D. R. Yoerger and J.-J. E. Slotine. Robust Trajectory Control of Underwater Vehicles. IEEE Journal of Oceanic Engineering, 10(4):462-470, Oct 1985. 